\title{
Nuclear Chemistry Progress Report
}

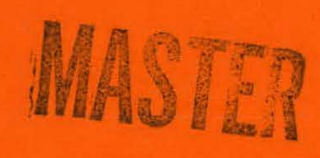

Oregon State University

An account of the research performed under USDOE Contract No. EY-76-S-06-2227-TA35 MT 2

September 1, 1978 to August 31, 1979

Walter D. Loveland

Principal Investigator 


\section{DISCLAIMER}

This report was prepared as an account of work sponsored by an agency of the United States Government. Neither the United States Government nor any agency Thereof, nor any of their employees, makes any warranty, express or implied, or assumes any legal liability or responsibility for the accuracy, completeness, or usefulness of any information, apparatus, product, or process disclosed, or represents that its use would not infringe privately owned rights. Reference herein to any specific commercial product, process, or service by trade name, trademark, manufacturer, or otherwise does not necessarily constitute or imply its endorsement, recommendation, or favoring by the United States Government or any agency thereof. The views and opinions of authors expressed herein do not necessarily state or reflect those of the United States Government or any agency thereof. 


\section{DISCLAIMER}

Portions of this document may be illegible in electronic image products. Images are produced from the best available original document. 
Nuclear Chemistry Progress Report

Oregon State University

An account of the research performed under USDOE Contract No. EY-76-S-06-2227-TA35 Mi

September 1, 1978 to August 37, 1979

Walter D. Lovel and

Principa? Investigator

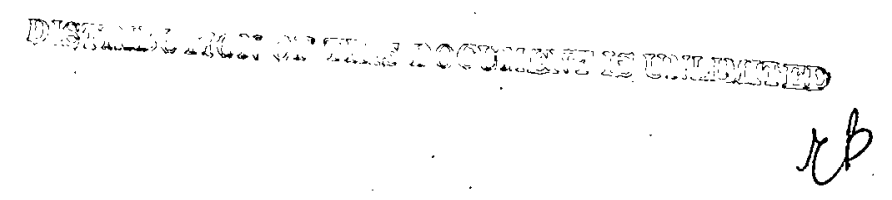




\section{Iable of Contents}

I. Introduction

II. Low Energy Heavy Ion Research

A. Superheavy Elements - A Crossroads . . . . . . . . . . . . . . . . 3

B. "Calibration" of ${ }^{44} \mathrm{SC}$ Isomer Ratio Measurements for Heavy Ion 9 Reactions and Its Imptications . . . . . . . . . . . . . .

C. Complete Fusion Studies in the ${ }^{48} \mathrm{Ca}+{ }^{248} \mathrm{Cm}$ Reaction........

III. Relativistic Heavy Ion Research

A. Interlaboratory Measurement of the Target Residue Recoil Properties

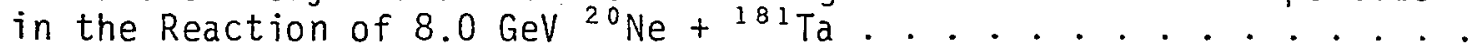

B. Measurement of Absolute Cross Sections for the Interaction of

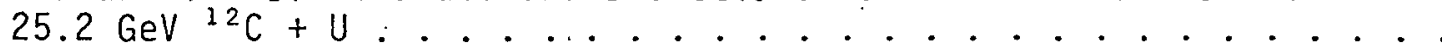

C. Radiochemical Studies of Product Mass and Charge Distributions in the Interaction of Relativistic Heavy Ions with Uranium . . . . . .

D. Product Recoil Properties in the Interaction of Relativistic Heavy Ions with Uranium . . . . . . . . . . . . . . . . . . . . . .

E. Near-Target Residues from the Peripheral Interaction of Relativistic Heavy Ions with Bismuth. . . . . . . . . . . . . . . . . . . . .

F. Target Residues from the Reaction of $8 \mathrm{GeV}^{20} \mathrm{Ne}$ with ${ }^{181} \mathrm{Ta}$ and ${ }^{197} \mathrm{Au}$

G. Energy Variation of Product Mass and Charge Distributions in the Interaction of Relativistic Heavy Ions with Ta and $U . . . . . . .$.

H. Product Mass and Charge Distributions in the interaction of $21 \mathrm{GeV}$

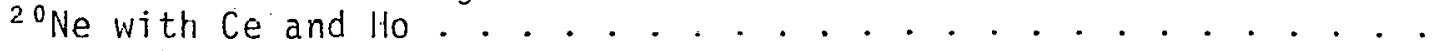

IV. Personnel . . . . . . . . . . . . . . . . . . . . . .

v. Publications . . . . . . . . . . . . . . . . . . . . . . 80

VI. Oral Presentations . . . . . . . . . . . . . . . . . . . . . 81 Appendix - Collected Reprints and Preprints . . . . . . . . . . . . 


\section{Introduction}

In this report, we summarize the highlights of the work done between 1 September, 1978 and 1 September, 1979 that was supported by USDOE Contract No. EY-76-S-06-2227-TA35-M1. The work reported herein is the result of a collaborative effort involving G. T. Seaborg and his research group and the nuclear chemists from Oregon State University and should be viewed as such. Each project discussed was the result of a joint effort of the two groups working together, interchanging roles in data acquisition and analysis. The individuals contributing to each project are listed at the end of each section with the names of the Oregon State scientists being underlined. Some of the work reported herein is in its preliminary stages and use of the data contained in these preliminary reports should be made after consultation with the appropriate authors.

The work described involves the study of low energy and relativistic heavy ion reactions. In the area of low energy heavy ion reactions, most of the effort went into a careful re-examination of the failure to synthesize superheavy elements using complete fusion reactions. The principal conclusion of this study was that the fission barriers of the superheavy elements (SHE's) must be $\leqslant 5-6 \mathrm{MeV}$, and thus the lonqest half 1 ife of a SHE $210^{5}$ years, a result precluding their observation in nature. Other studies which attempted to measure the extent to which complete fusion occurred in the ${ }^{48} \mathrm{Ca}+{ }^{248} \mathrm{Cm}$ reaction were alsu begun.

In the area of relativistic heavy ion (RHI) reaction studies, the most interesting results came in ${ }^{(a)}$ our studies of the energy dependence of the product mass and charge distributions resulting from the interaction of RHI's with uranium and ${ }^{(b)}$ studies of the recoil properties of the target residues produced in the reaction of RHI's with heavy targets. The yield of the large col1ision residues $(A=150-200)$ so copiously produced in the interaction of $25 \mathrm{GeV}{ }^{12} \mathrm{C}$ with ${ }^{230} \mathrm{U}$ is dramatically reduced in the reaction of $8 \mathrm{GeV} 20 \mathrm{Ne}$ with ${ }^{238} \mathrm{U}$. The study of the recoil properties of the target fragments from the reaction of $8 \mathrm{GeV}{ }^{20} \mathrm{Ne}+\mathrm{Ta}$ and $4.8 \mathrm{GeV}{ }^{12} \mathrm{C}+{ }^{238} \mathrm{U}$ shows that the 1 ightest products $(A<40)$ and the heavy rare earth products $(A=140-160)$ have much higher momenta imparted to them by the incident heavy ion than corresponding products produced by the interaction of relativistic protons with the same nuclei. Both these 
- results would seem to indicate that detailed studies of the lightest and heaviest target fragments from RHI induced reactions offer us the possibility of studying "hard" nucleus-nucleus collisions and gaining insight into the unique aspects of RHI collisions. As part of our systematic examination of the product mass and charge distributions from the interaction of RHI's with heavy nuclei, we have measured such distributions from the interaction of a fixed energy heayy ion ( 8 and $21 \mathrm{GeV}{ }^{20} \mathrm{Ne}$ ) with a wide range of targets ( $\mathrm{Ce}, \mathrm{Ho}, \mathrm{Ta}, \mathrm{Au}, \mathrm{Bi}, \mathrm{U}$ ) and measured these distributions as a function of projectile energy ( $5 \mathrm{GeV}, 8 \mathrm{GeV}$, $21 \mathrm{GeV}{ }^{20} \mathrm{Ne}$ ) with a few targets $(\mathrm{Ta}, U)$. Our fission track detector studies of the fission product angular distributions from the interaction of RHI's with $U$ have continued and show an interesting energy dependence of the track distributions. 
II. Low Energy Heavy Ion Reactions

II.A. - Superheavy Elements: A Crossroads

$\because$ For many years nuclear scientists believed that the periodic table had been extended nearly to its limit, defined as the point where the number of protons in the nucleus, and consequently the repulsion between them becomes so large that the cohesive nuclear forces cannot hold the nucleus together and the nucleus therefore undergoes very rapid spontaneous fission decay. This idea was based on the observation of shorter and shorter spontaneous fission half-lives as the atomic number of the nucleus increased.

In the period from 1966 to 1972, a number of calculations (1) based on modern theories of nuclear structure showed that in the region of proton number $Z=114$ and neutron number $N=184$ the ground states of nuclei were stabilized against fission. Some of these detailed calculations even suggested that the half-lives of some of these superheavy nuclei might be on the order of the age of the universe, and this stimulated a great effort to observe these "missing elements" in nature. The superheavy elements (SHE's) were predicted to form an island of relative stability extending both above and belo: $Z=114$ and $N=184$ and separated from the peninsula of known nuclei by a sea of instability.

Table II-A-I is a summary of recent attempts to systhesize SHE's in nuclear reactions, utilizing the complete fusion of two heavy ions. The energetics of the reactions, fission barrier heights, and neutron binding energies were taken from appropriate recent calculations (10). Since the sought-after SHE is initially produced as an excited compound nucleus, its survival requires the loss of its excitation energy by the emission of neutrons in competition with the much more probably fission process (which will destroy the superheavy nucleus if it occurs). A simple estimate of the survival rate of the superheavy nuclei former in these reactions was made by using expressions for Tevel densities of a Fermi gas, including consideration of the effect of angular momentum on SHE survival (13).

Because of the promising character of the ${ }^{248} \mathrm{Cm}+48 \mathrm{C}$. a reaction for synthesizing superheavy nuclei and the apparent failure of this reaction to do $s n$, it. hehonves us to examine this system in greater detail to see why production of SHE's was not observed. 
What would we have expected the formation cross section for superheavy nuclei to be in this reaction? An estimate of the cross section for the fusion of ${ }^{48} \mathrm{Ca}$ and $248 \mathrm{Cm}$ might be $\sigma_{F} \geq 10^{-27}$ square centimeters based on the observation (14) of the production of the complete fusion product $25 \frac{5}{1}{ }_{2}$ No with a cross section of $3 \times 10^{-30} \mathrm{~cm}^{2}$ from the similar ${ }^{48} \mathrm{Ca}+208 \mathrm{pb}$ fusion reaction. Using the method of Table II-A-l to estimate survival probabilities, one calculates a survival probability of $210^{-5}$ for $254 \mathrm{No}$ nuclei, implying a complete fusion cross section of $2300 \times 10^{-27} \mathrm{~cm}^{2}$. From this number and the systematics of complete fusion cross sections, we extrapolate a value of $\sigma_{C F} \geq 10^{-27} \mathrm{~cm}^{2}$ for the $48 \mathrm{Ca}+248 \mathrm{Cm}$ reaction. In addition, we note that in the reaction of $40 \mathrm{Ar}$ and $48 \mathrm{Ca}$ with $238 \mathrm{U}$, products were observed $(15,16)$ (with a production cross section $\geq 60 \times 10^{-27} \mathrm{~cm}^{2}$ ) that appear to have resulted from the fusion of the ${ }^{40} \mathrm{Ca}$ or $48 \mathrm{Ar}$ and the $238 \mathrm{U}$ nucleus followed by fission. (These products have excitation functions and angular distributions characteristic of the fusion-fission process). Since the only definitive signature of the complete fusion process in the $48 \mathrm{Ca}+248 \mathrm{Cm}$ reaction is the detection of SHE's, it is possible that the reacting ions did not actually fuse - a possibility suggested by some calculations (17) - but in view of the evidence cited above, we will proceed under the assumption that some fusion (cross. section $\geq 10^{-27} \mathrm{~cm}^{2}$ ) did take place in the ${ }^{48} \mathrm{Ca}+243 \mathrm{Cm}$ reaction.

A schematic representation of the de-excitation of any 296116 compound nuclei formed in the ${ }^{48} \mathrm{Ca}+248 \mathrm{Cm}$ reaction is shown in Table II-A-2, where we used two different estimates of the reaction energetics and fission barrier heights to calculate the survival rates of the superheavy nuciei. The estimates used are those of Fiset and Nix (12) and those of Randrup et al. (11), the former are similar to most theoretical calculations done in the period from 1966 to 1972 , and the latter represent the more recent approach. The "experinlental" upper limit on the SHE survival rate in this reaction can be calculated as the ratio of the SHE production cross section upper limit to the complete fusion cross section; that is, $5 \times 10^{-35} / 10^{-27}=5 \times 10^{-8}$. Clearly the calculations based on the older, more optimistic barriers and energetics grossly overestimate the survival probabilities in this reaction, giving values approaching unity. The calculations based on the more recent, pessimistic barriers and energetics are consistent with the data. The calculations based on the barriers and energetics of Fiset and Nix can be brought into agreement with the observed upper limits to the cross sections for SHE production by using values for the fission barrier heights that are 4 to 5 MeV 
lower than those originally predicted. The overall cross section for the production of detectable superheavy nuclei would be $10^{-27} \times 10^{-11} \leq 10^{-38} \mathrm{~cm}^{2}$, using the barriers of Randrup et al. One can appreciate the minuscule magnitude of these cross sections by realizing that under the most favorable experimental conditions available today, a production cross section of $10^{-35} \mathrm{~cm}^{2}$ would correspond to the production of one to three SHE atoms per day of irradiation.

Thus the failure to observe SHE's in this reaction seems to indicate that the fission barriers of these elements are considerably lower than those reported earlier (1, 12). This observation has certain qualitative consequences. If one accepts the calculations of Randrup et al. (11) as correctly descrihing the properties of the superheavy nuclei (which is consistent with the experimental data for the ${ }^{48}$.a $+{ }^{248} \mathrm{~cm}$ reartion), then, one concludes that the longest. halflife of a superheavy nucleus is $210^{5}$ years - which precludes their observation in terrestrial matter or any object whose age significantly exceeds $10^{5}$ years, such as cosmic radiation. (This, of course, does not preclude observation of fossil remnants of extinct SHE's, such as decay products or fission tracks.) At the same time, one must be careful to note that the experimental results only test the cumulative survival probabilities, not the topology of the superheavy island. Thus we do not know whether the island of stability has a structure like the Matterhorn, steeply falling. into the sea of instability as $N$ ierreases from 184, as suggested by the calculations of Randrup et al., or whether it is a lesser peak with a broad base extending to significantly lower values of $N$, thus resembling the legendary home of Satan in the San Francisco Bay area, Mnunt. Diablo, as would be suggested by the Fiset and Nix topology appropriately lowered to fit experimental data.

(G. T. Seaborg,* W. Lovcland, and D. U. Morrissey.*) 
TABLE II -A-] Attempts to synthesize superheavy elements by using complete fusion reactions.

\begin{tabular}{|c|c|c|c|c|c|}
\hline \multirow[b]{2}{*}{$\begin{array}{l}\text { Reaction } \\
\text { studied }\end{array}$} & \multicolumn{3}{|c|}{ Compound nucleus } & \multirow[b]{2}{*}{$\begin{array}{l}\text { Observed upper-limit cross } \\
\text { section for SHE production } \\
\text { for indicated half-life } \\
\qquad\left(\mathrm{cm}^{2}\right)\end{array}$} & \multirow[b]{2}{*}{$\begin{array}{l}\text { Refer- } \\
\text { ence }\end{array}$} \\
\hline & $A_{z}$ & $\begin{array}{l}\text { Mean } \\
\text { exci- } \\
\text { tation } \\
\text { energy } \\
(\mathrm{MeV})\end{array}$ & $\begin{array}{l}\text { Pre- } \\
\text { dicted } \\
\text { survival } \\
\text { proba- } \\
\text { bility }\end{array}$ & & \\
\hline \multicolumn{6}{|c|}{ Class 1: Compound nuclei with low survival probabilities } \\
\hline $\begin{array}{l}232 \mathrm{Ch}+48 \mathrm{Ca} \\
231 \mathrm{~Pa}+48 \mathrm{Ca} \\
233 \mathrm{U}+48 \mathrm{Ca} \\
248 \mathrm{Cm}+40 \mathrm{Ar} \\
242 \mathrm{Pu}+48 \mathrm{Ca} \\
243 \mathrm{Am}+{ }^{48} \mathrm{Ca}\end{array}$ & $\begin{array}{l}280110 \\
279711 \\
281112 \\
288114 \\
290714 \\
291715\end{array}$ & $\begin{array}{l}44.5 \\
34 \\
33 \\
45 \\
43.5 \\
47\end{array}$ & $\begin{array}{l}10^{-21} \\
10^{-17} \\
0^{*} \\
0^{*} \\
0^{*} \\
0^{*}\end{array}$ & 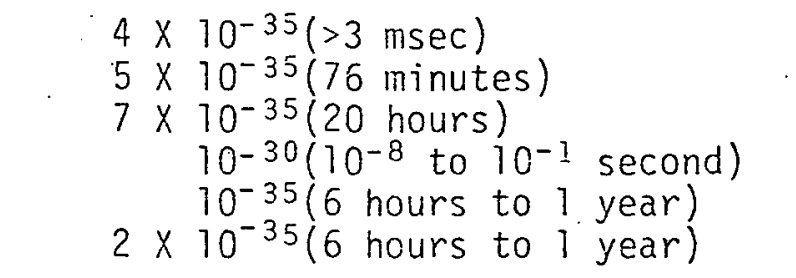 & $\begin{array}{l}(9) \\
(9) \\
(9) \\
(2) \\
(3) \\
(3)\end{array}$ \\
\hline \multicolumn{6}{|c|}{ Elass 2: Smal1 probability of forming compound nuclei } \\
\hline $\begin{array}{l}208 \mathrm{~Pb}+84 \mathrm{Kr} \\
2 \cdot 38 \mathrm{U}+68 \mathrm{Zn} \\
232 \mathrm{Th}+76 \mathrm{Ge} \\
242 \mathrm{Pu}+68 \mathrm{Zn} \\
238 \mathrm{U}+76 \mathrm{Ge} \\
243 \mathrm{Am}+68 \mathrm{Zn} \\
246 \mathrm{Cm}+68 \mathrm{Zn} \\
232 \mathrm{Th}+84 \mathrm{Kr}\end{array}$ & $\begin{array}{l}292718 \\
305722 \\
308722 \\
310724 \\
314724 \\
311725 \\
314726 \\
316726\end{array}$ & $\begin{array}{c}25.5 \\
47 \\
32 \\
45 \\
68 \\
39 \\
34 \\
51\end{array}$ & $\begin{array}{l}20\left(10^{-9}\right)^{\dagger} \\
1.0 \\
1.0 \\
0.9 \\
3 \times 10^{-2} \\
0.9 \\
0.3 \\
<10^{-14}\end{array}$ & $\begin{array}{l}10^{-30}\left(>6 \times 10^{-7} \text { second }\right) \\
10^{-30}\left(10^{-9} \text { sccond to } 1 \text { year }\right) \\
10^{-34}(5 \mathrm{msec} \text { to } 1 \text { year }) \\
10^{-30}\left(10^{-9} \text { second to } 1 \text { year }\right) \\
10^{-33}(5 \mathrm{msec} \text { to } 1 \text { year }) \\
2 \times 10^{-32}\left(10^{-9} \text { second to } 1 \text { day }\right) \\
10^{-30}\left(10^{-9} \text { second to } 1 \text { year }\right) \\
5 \times 10^{-30}\left(>5 \times 10^{-7} \text { second }\right)\end{array}$ & $\begin{array}{l}(4) \\
(5) \\
(6) \\
(5) \\
(6) \\
(5) \\
(5) \\
(4)\end{array}$ \\
\hline \multicolumn{6}{|c|}{ Class 3: Compound nuclei with possible survival } \\
\hline${ }^{246} \mathrm{Cm}+{ }^{48} \mathrm{Ca}$ & 294716 & 40 & $\begin{array}{l}<5 \times 10^{-16} \\
\left(10^{-11}\right)^{+}\end{array}$ & $2 \times 10^{-35}(6$ hours to 1 year $)$ & (3) \\
\hline${ }^{248} \mathrm{Cm}+{ }^{48} \mathrm{Ca}$ & 296716 & 44 & $\begin{array}{l}<4 \times 10^{-11} \\
\left(10^{-5}\right)^{++}\end{array}$ & $5 \times 10^{-35}$ ( 6 hours to 1 year $)$ & $\begin{array}{l}(3,7) \\
(8)^{-1}\end{array}$ \\
\hline
\end{tabular}

* Nuclei whose survival rate is exactly zero represent cases in which some member of the neutron emission chain has a nonexistent fission barrier.

The cumulative survival rate for these nuclei up to the last step in the de-excitation process is given in parenthesis. In the last step of the de-excitation process, the excitation energy is at or below the neutron binding energy and well above the fission barrier. The result of this circimstance is a fission catastrophe in which nearly all the nuclei fission.

${ }^{+}$See text for discussion. 
TABLE II-A-2 De-excitation of superheavy element precursors from the ${ }^{48} \mathrm{Ca}+{ }^{248} \mathrm{Cm}$ reaction

\begin{tabular}{|c|c|c|c|c|c|c|}
\hline \multirow[t]{2}{*}{ Nucleus } & \multirow{2}{*}{$\begin{array}{l}\text { Excitation } \\
\text { energy } \\
\text { (MeV) }\end{array}$} & \multicolumn{2}{|c|}{$\begin{array}{r}\text { Based on } \\
\text { Randrup et a 1. (11) }\end{array}$} & \multicolumn{3}{|c|}{$\begin{array}{l}\text { Based on } \\
\text { Fiset and } \operatorname{Mix}(12) \\
\end{array}$} \\
\hline & & $\begin{array}{l}\text { Fission } \\
\text { barrier } \\
\text { height } \\
\text { (MeV) }\end{array}$ & $\begin{array}{l}\text { Fraction } \\
\text { surviving } \\
\text { fission } \\
(\%) \\
\end{array}$ & $\begin{array}{l}\text { Fission } \\
\text { barrier } \\
\text { height } \\
\text { (Mey) }\end{array}$ & $\begin{array}{l}\text { Fraction } \\
\text { surviving } \\
\text { fission } \\
(\%) \\
\end{array}$ & \\
\hline 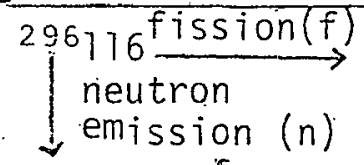 & 44 & 5.7 & 18 & 11.0 & 98 & \\
\hline $\int_{n}^{295716-f}$ & 34 & 5.9 & 23 & 10.5 & 99 & \\
\hline $\ln _{n}^{294} 716 \underset{f}{\longrightarrow}$ & 26 & 4.1 & 1.4 & 10.0 & 99 & \\
\hline $\operatorname{lom}_{n}^{2 \dot{9}^{3}} 116 \ldots$ & 16 & 3.5 & 1.4 & 9.6 & 100 & \\
\hline $292116 \_f$ & 8 & 2.9 & $5 \times 10^{-4}$ & 9.3 & 100 & \\
\hline $\begin{array}{l}\gamma, n \\
\text { Predicted cum } \\
\text { survival prob }\end{array}$ & $\begin{array}{l}\text { ative } \\
\text { ility }\end{array}$ & & $<4 \times 10^{-11}$ & & 0.96 & \\
\hline
\end{tabular}




\section{REFERENCES}

Condensed from Science 203, 711 (1979)

*.Permanent address: Lawrence. Berkeley, Laboratory, Berkeley, CA 94720.

1. C. E. Bemis, Jr., and J. R. Nix, Comments Nucl. Part. Phys. I, 65 (1977).

2. H. R. Bowman, R. C. Gatti, R. C. Jared, L. G. Moretto, W. J. Swiatecki, S. G. Thompson, Lawrence Berkeley Lab. Rep. UCRL-17989 (1967).

3. Y. T. Oganession et al., Nuci. Phys. A 294, 213 (1978).

4. P. Colombani, B. Gatty, J. C. Jacmart, M. Lefort, J. Peter, M. Riou, C. Stephen, X. Tarrago, Phys. Lett $\underline{B} 42,208$ (1972).

5. A. Pleve, A. G. Demin, V. Kush, M. B. Miller, N. A. Danilov, Sov. J. Nucl. Phys. 19, $123(1974)$.

6. G. N. Flerov et al., ibid., p. 247.

7. J. D. Illige, E. K. Hulet, J. M. Nitschke, R. T. Dougan, R. W. Lougheed, A. Ghiorso, J. H. Landrum, Phys. Lett. B 78, 209 (1978).

8. E. K. Hulet et al., Phys. Rev. Lett. 39, 385 (1977); R. J. Otto, D. J. Morrissey, D. Lee, A. Ghiorso, J. M. Nitschke, G. T. Seaborg, J. Inorg. Nuc 1. Chem. 40, 589 (1978).

9. Yu. Ts. Oganessián et a1., Nucl. Phys. A 294, 213 (1978).

10. For nuclei with $Z \leq 120$, the droplet model masses (W. D. Myers, The Droplet Model of Atomic Nuclei (Plenum, New York, 1978)) were corrected by using the shell corrections in (11) to give reaction energetics and neutron binding energies. For $Z>120$, the energetics and barriers were taken from (12).

11. J. Randrup, S. E. Larsson, P. Moller, A. Sobiczewski, A. Lukasiak, Phys. Scr. 10R, $60(1974)$.

12. E. 0. Fiset and J. R. Nix, Nucl. Phys. A 193, 647 (1972).

13. The formalism used is outlined by R. Vandenbosch and J. R. Huizenga (Nuclear Fission (Academic Press, New York, 1973), pp. 233-250). Details of its applications are given by W. Loveland (Oregon State University. Rep. RL0-2227-TA35 (7978)). 
Each emitted neutron was assumed to remove an excitation energy equal to the sum of the neutron binding energy and twice the nuclear temperatures $\left(B_{n}+2 T\right)$. Values of the mean square projection of the nuclear angular momentum on the nuclear symmetry axis, $k_{0}{ }^{2}$, were taken from typical values for transuranium nuclei ( $p .202$ in Vandenbosch and Huizenga); the fission level density parameter af was set equal to $1.1 a_{n}=1.1(\mathrm{~A} / 8)$, where $a_{n}$ is the neutron level density parameter and $A$ is the nuclide mass number.

14. J. M. Nitschke, R. E. Leber, M. J. Nurmia, A. Ghiorso, Lawrence Berkeley Lab. Rep. LBL-65.34 Rev. (1978).

15. R. J. Otto, D. J. Morrissey, G. T. Seaborg, W. D. Loveland, Z. Phys. A 287. 97 (1978).

16. M. de Saint Simon, R. J. Otto, G. T. Seaborg, Phys. Rev. C 18, 1651 (1978).

17. A. J. Sierk, in Proceedings of the International Symposium on Supcrheavy Elements, M. K. Lodhi, Ed., (Pergamon, 1979).

II-B- "Calibration of ${ }^{4} \mathrm{SC}$ Isomer Ratio Measurements for Heavy Ion Reactions

Measurements of the primary product angular momenta in studies of heavy ion nuclear reactions can yield important information about the mechanism(s) operating in these reactions. For example, one might expect that complete fusion and deep inelastic scattering might lead to rather different product angular momenta. Furthermore, the comparison of the measured product angular momenta with dynamical calculations should give insight into the detailed nature of. the forces operating in these reactions. The angular momenta of fiscinning systems produced by deep inelastic scattering has been deduced from the measurement of fragment angular correlations $(1,2)$ while gamma ray multiplir.ities measured in coincidence with fragments from quasi-elastic and deep inelastic scattering $(3,4,5,6)$ have yielded additional information about the distribution of angular momenta in these reactions. An extensive third source of information about these angular momenta that is complementary to the previous two methods has not been utilized. This third source of information is the one hundred or so independent yield isomer ratio measurements from radiochemical studies of low energy and relativistic heavy ion reactions. 
The problem has been to interpret these measurements in terms of the primary product angular momenta preceding neutron and gamma ray emission. Simple and very sophisticated theoretical frameworks exist to follow these neutron emission and gamma ray cascades to predict the product isomer ratios. Each formalism has certain unknown parameters which must be fixed to allow the calculations to be done. During the past year, we have begun a set of "isomer ratio" calibration experiments. The isomeric pair ${ }^{44} \mathrm{~m} S C(I=6+),{ }^{449} \mathrm{SC}(I=2+)$ was produced in the ${ }^{29} \mathrm{Si}\left({ }^{18} 0, \mathrm{p} 2 \mathrm{n}\right){ }^{44} \mathrm{Sc}$ reaction. The isomer ratio, om/og, was measured for ${ }^{180}$ energies from $30-80 \mathrm{MeV}$ using the LBL $88^{\prime \prime}$ cyclotron ${ }^{18} 0$ beam. Thin targeis of ${ }^{25} \mathrm{Si}$ (95\% isotopically pure) in the oxide form were prepared on tantalum foil backings. This allowed off-line gamma ray spectroscopic identification of the reaction products without interference from activities produced by reaction with the catcher foil when the beam energy was below nomeV. For the highest beam energy, $80 \mathrm{MeV}$, a chemical separation of Sc isotopes from the catcher foil was necessary. The decay of ${ }^{4} 49_{S C}$ goes $99.89 \pm 0.01 \%^{\prime}$ via a 1157 keV gamma transition while the ${ }^{44 \mathrm{~m}_{\mathrm{Sc}} \text { goes } 86.6 \pm 0.2 \%}{ }^{7}$ via a $271 \mathrm{keV}$ internal gamma transition followed by the decay of the ground state. These gamma lines were measured using a $\mathrm{Ge}(\mathrm{L} i$ ) detector and were, more or less, the only lines in the spectrum.

The production cross sections $(\sigma 6+$ and $\sigma 2+)$ were calculated from the yield data and the ratio $(\sigma 6+/ \sigma 2+)$ was thus determined for each different projectile energy. The values of these ratios are shown in Figure II-B-1. The ratio, $(\mathrm{m} / \mathrm{og})$ increases from 0.9 at $30 \mathrm{MeV}$ to 4.7 at $70 \mathrm{MeV}$.

Calculations of the root mean square angular momentum of the initial compound. nucleus were made using a Bass ${ }^{8}$ potential and a harmonic oscillator potential due to Thomas. 9 The results are insensitive to the choice of potential. The dependence of the ${ }^{4} \mathrm{SC}$ isomer ratio on initial compound nucleus angular momentum is shown in Figure II-B-2. The double valued aspect of the isomer ratio at high $1 \mathrm{rms}$ is believed to be due to the onset of direct reaction processes contributing to the ${ }^{29} \mathrm{Si}\left({ }^{18} 0\right.$, pxii) reaction.

We have attempted to fit this data into a standard Vandenbosch - Haizenga isomer ratio calculation. 10 We used Bjorklund - Fernbach neutron transmission coefficients, ${ }^{11}$ proton transmission coefficients of Mani, ${ }^{12}$ and a superconductor model for the spin cutoff parameter. 13 The $y$-ray cascade was assumed to contain 1 or 2 El transitions with the rest being E2 transitions. The results of the calculations are also shown in Figure II-B-2 and the agreement between calculation and experiment appears to be satisfactory. We are exploring the use of more sophisticated prescriptions for describing the de-excitation cascade to see if they also can be "calibrated" using this data.

(K. Aleklett, D. J. Morrissey, W. Loveland, P. McGaughey and G. T. Seaborg) 


\section{REFERENCES}

1. P. Dyer, R. J. Puigh, R. Vandenbosch, T. D. Thomás, and M. S. Zisman, Phys. Rev. Lett. 39,392 (1977).

2. G. J. Wozniak, R. P. Schmitt, P. Glassel, R. C. Jared, G. Bizard, and L. G. Moretto, Phys. Rev. Lett. $\underline{\text { 40, }} 1436$ (1978).

3. P. Glassel, R. S. Simon, R. M. Diamond, R. C. Jared, I. Y. Lee, L. G. Moretto, J. O. Newton, R. Schmitt, and F. S. Stephens, Phys. Rev. Lett. 38, 331 (1977).

4. M. Berlanger, M. A. Deleplanque, C. Gerschel, F. Hannappe, M. Leblanc, J. F. Mayauit, C. Ngo, D. Paya, N. Perrin, J. Peter, B. Tamain, and L. Vaientin, J. Phys. Lettres 37, L323 (1976).

5. M. M. Aleonard, G. J. Wozniak, P. Glassel, M. A. Deleplanque, R. M. Diamond, L. G. Moretto, R. P. Schmitt, and F. S. Stephens, Phys. Rev. Lett. 40, 62? (1978).

6. P. R. Christensen, F. Folkmann, 0. Hansen, 0. Nathan, N. Trautner, F. Videbaek, S. Y. van der Werf, H. C. Britt, R. P. Chestnut, H. Freisleben and F. Puhthofer, Phys. Rev. Lett. 40,1245 (1978).

7. C. M. Lederer and V. S. Shirley, Table of Isotopes, 7th Ed. (Wiley, New York, $19 \%$ ).

8. R. Bass, Phys. Lett. 47B, 139 (1973)

9. T. D. Thomas, Phys. Rev. 116, 703 (1959)

10. W. L. Hafner, Jr., J.R. Huizenga, and R. Vandenbosch. Argonne National Laboratory Report ANL-6662 (1962)

11. E. H. Auerbach and F. G. 1. Perey, Brookhaven National Laboratory Report. BNL -765

12. G. S. Mani, M. A. Melkunuff and I. Iori, Saclay Report CEA-2379 (1963)

13. H. Vonach, R. Vandenbosch and J. R. Huizenga, Nuct. Phys. 60, 70 (1964) 
Figure II-B-1 The ratio $(\sigma 6+/ \sigma 2+)$ for the isomeric pair $44 m$,gSc as function of the ${ }^{18} 0$ beam energy. XBL -2427 


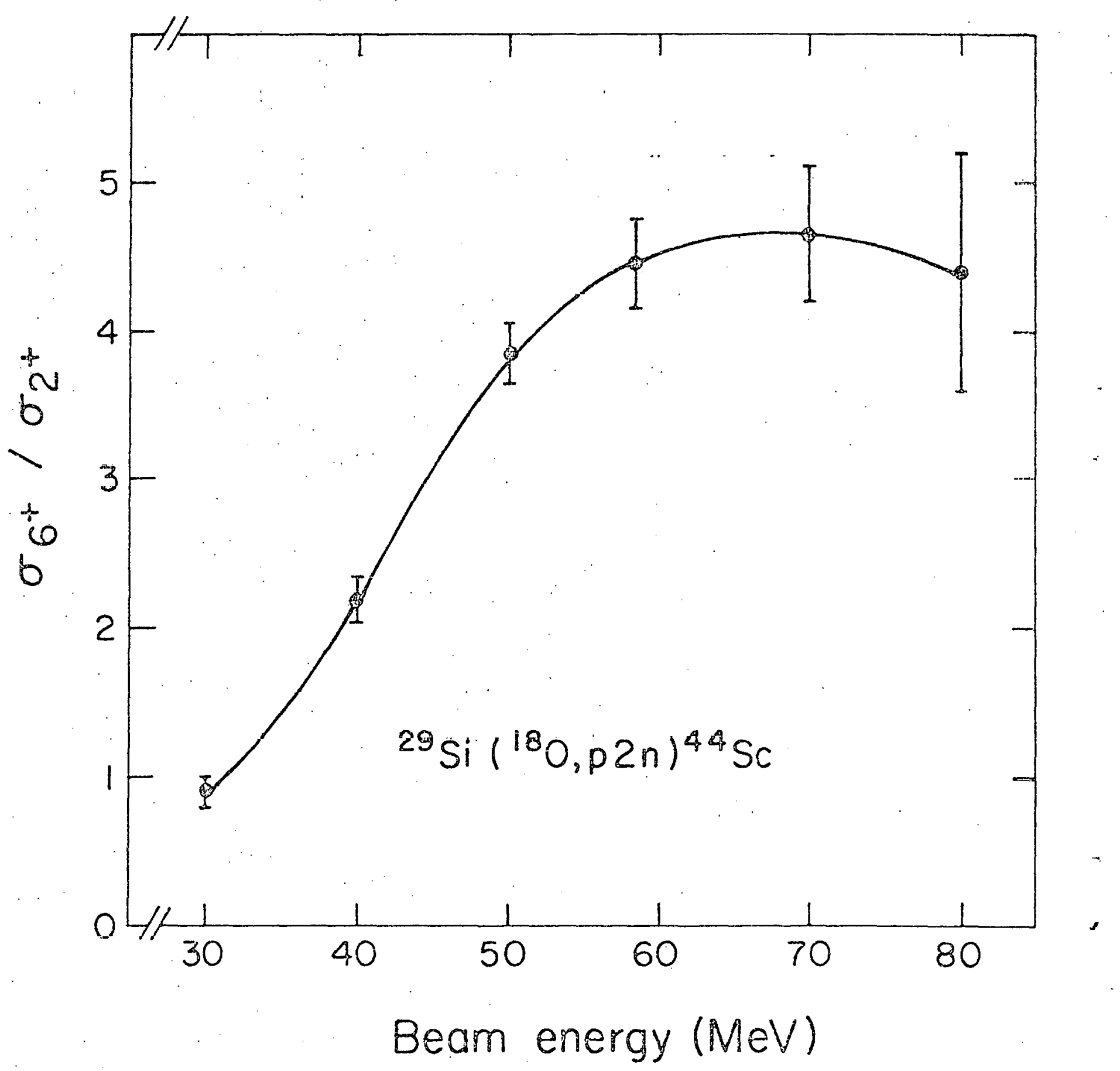




\section{,}

?

Figure II-B-2 ${ }^{44} \mathrm{SC}$ isomer ratio as a function of the root mean square angular momentum of the fissioning system. 


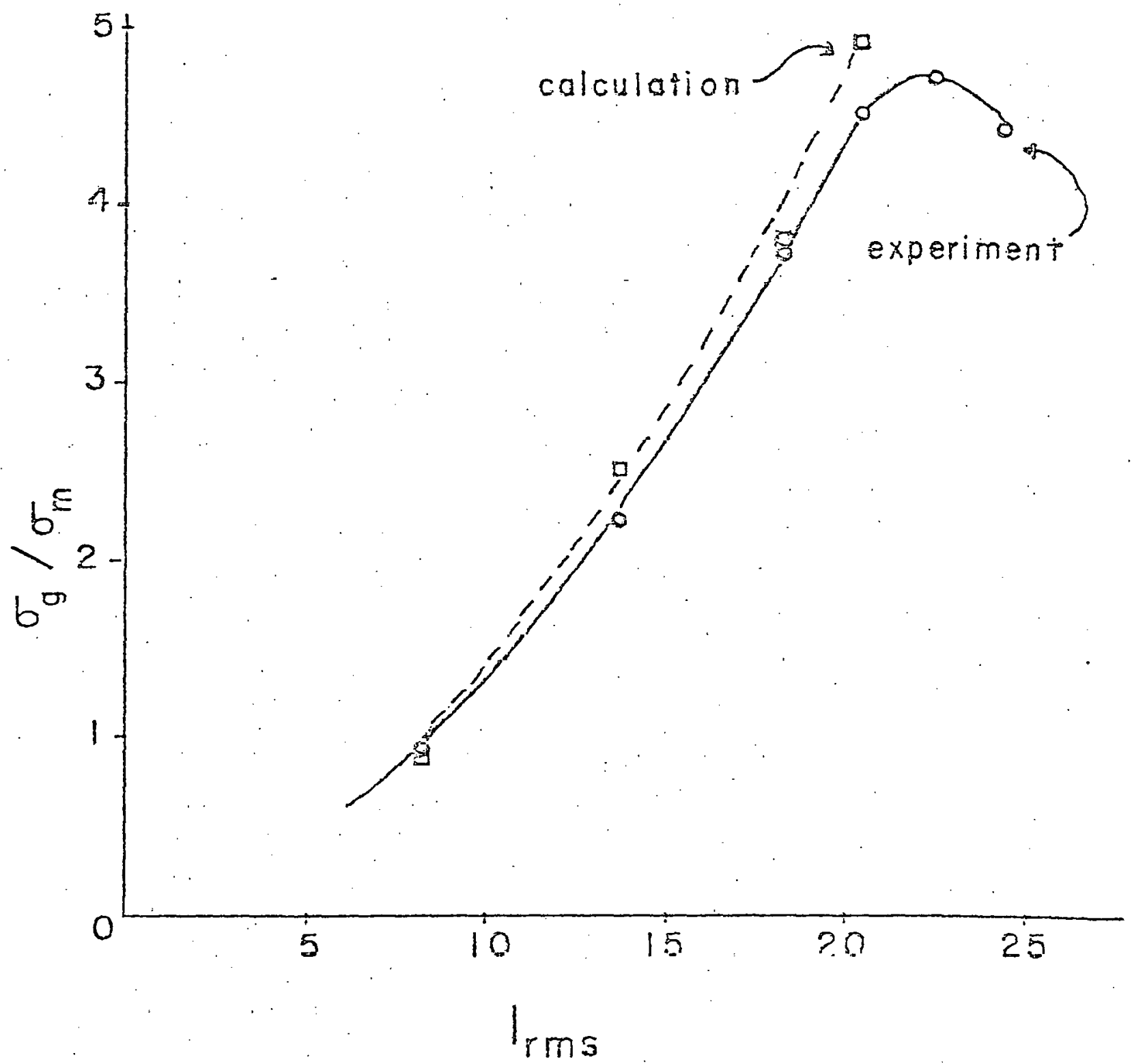




\section{II-C- Complete Fusion Studies in the ${ }^{48} \mathrm{Ca}+{ }^{248} \mathrm{Cm}$ Reaction}

A crucial component of the attempt to synthesize superheavy elements (SHE's) using the ${ }^{48} \mathrm{Ca}+{ }^{248} \mathrm{Cm}$ reaction is the assumption, as discussed in Section II-A of this report, that at bombarding energies low enough for SHE survival that complete fusion of the ${ }^{48} \mathrm{Ca}$ and ${ }^{248} \mathrm{Cm}$ nuclei occurs. Since no evaporation residues (i.e., SHE's) were found, one is left to make arguments based upon anaiogies to other reactions involving ${ }^{48} \mathrm{Ca}$ ions and heavy targets, as was done in Section II-A. We thought it to be important therefore to undertake more direct measurements of the ${ }^{48} \mathrm{Ca}+{ }^{248} \mathrm{Cm}$ reaction to see if we could uncover other experimental evidence that complete fusion was occurring.

We ruled out use of the shape of the "fusion-fission" component of the product mass distribution or the angular distribution of these products as evidence for the occurence of complete fusion because we had shown earlier that it was very difficult to distinguish between complete fusion followed by fission and a deep inelastic process in which the projectile and target nuclei momentarily ." united, exchanged nucleons, underwent a few rotations, and broke apart without ever reaching a deformation of the united system inside of the fission saddle point. We, instead, chose to attempt to measure the excitation functions of selected "fusion-fission" products of varying mass in this reaction. We were inspired in this effort by the work of St. Simon et al. ${ }^{2}$ who were able to quantitatively separate the aforementioned processes as they affected the yield of iodine isotopes in the ${ }^{40} \mathrm{Ar}+{ }^{238} \mathrm{U}$ reaction.

With the cooperation of $A$. Ghiorso, we bombarded a $0.5 \mathrm{mg} / \mathrm{cm}^{2}{ }^{243} \mathrm{CmF}_{3}$ target with $254 \mathrm{MeV}$ and $278 \mathrm{MeV}{ }^{48} \mathrm{Ca}$ ions. Atoms recoiling from the target in an angular range of $6-45^{\circ}$ were collected with $A 1$ catcher foils and the bromine, iodine and gold isotopes were chemically separated ${ }^{3}$ from the recoil catchers and the radioactivities were measured using $\gamma$-ray spectroscopy. Analysis of this data is in progress. We hope to be able to complete this excitation function study in the future.

(W. Loveland, R. J. Otto, D. J. Morrissey, and G. T. Seaborg) 


\section{REFERENCES}

1. R. J. Otto, D. J. Morrissey, G. T. Seaborg, and W. D. Loveland, Z. Physik, A287, 97 (1978).

2. M. de Saint-Simon, R. J. Otto, and G. T. Seaborg, Phys. Rev. C18, 1651 (1978).

3. J. V. Kratz, J. 0. LiTjenzin and G. T. Seaborg, Inorg. Nucl. Chem. Lett., 10, 951 (1974).

III. Relativistic Heavy. Ion Reactions.

-I-I-I. A-Interlaboratory Measurement of the Target Residue Recoil Properities in the Reaction of $8.0 \mathrm{GeV}{ }^{20} \mathrm{Ne}+{ }^{131} \mathrm{Ta}$

Studies of target fragmentation in relativistic heavy ion (RHI) reactions have shown that the product mass and charge distributions resulting from relativistic heavy ion induced reactions are generally similar to those observed in reactions induced by protons of the same total energy. ${ }^{1,2}$ Furthermore, Kaufman et al. 3 have observed that the target residue recoil properties from the reaction of $25 \mathrm{GeV}{ }^{12} \mathrm{C}$ (i.e., $2.1 \mathrm{GeV} / \mathrm{amu}$ ) with ${ }^{197} \mathrm{Au}$ are very similar to those properties observed in the reaction of $28 \mathrm{GeV}$ protons with ${ }^{197} \mathrm{Au}$ and differ greatly from the residue recoil properties observed in the reaction of 3 GeV protons with ${ }^{197} \mathrm{Au}$. With these ideas in mind, we thought it would be interesting to measure the target residue recoil properties from reactions induced by lower energy (i.e., <25 GeV) heavy ions, since it is well known that the maximum momentum transfer occurs in the reaction of $1-3 \mathrm{GeV}$ protons with heavy targets.'

Following preliminary observations by our group, we collaborated with J. B. Cumming and P. E. Haustein of the Brookhaven National Laboratory and S. B. Kaufman, E. P. Steinberg and B. D. Wilkins of the Argonne National Laboratory in a joint measurement of the target residue recoil properties in the reaction of $8.0 \mathrm{GeV}{ }^{20} \mathrm{Ne}$ with ${ }^{181} \mathrm{Ta}$. In a single simultaneous bombardment at the Bevalac, each group irradiated its own thick target-thick catcher recoil experiment and analyzed the radioactivities induced in the foils at their own laboratories. The quantities determined from this analysis are $F / B$ and the approximate range $2 \omega(F+B)$ where $W$ is the target thickness and $F$ and $B$ are the fractions of a given radionuclide activity that recoiled out of the target

foil in the forward and backward directions, respectively (see Fig. III-A-1) Comparison of the data in Fig. III-A-1 with preliminary results of the ANL group 
shows the data sets agree within experimental error. Furthermore, comparison with the data of Bächman et al. ${ }^{5}$ or the data of reference 3 shows the ranges of the target residue fragments to be similar to those observed in the reaction of energetic protons with heavy non-fissionable targets but the fragment $(F / B)$ ratios are much larger in the reaction of $8.0 \mathrm{GeV}<\mathrm{vNe}$ with $131 \mathrm{Ta}$ than those observed for the reaction of energetic protons with ${ }^{131} \mathrm{Ta}$ or ${ }^{197} \mathrm{Au}$. Furthermore, the (F/B) ratios from the reaction of $8.0 \mathrm{GeV}{ }^{20} \mathrm{Ne}$ with ${ }^{181} \mathrm{Ta}$ are much larger than those observed in the reaction of $25 \mathrm{GeV}{ }^{12} \mathrm{C}+{ }^{197} \mathrm{Au} .^{3}$

These data were analyzed using a two step vector model ${ }^{6}$ in which the velocity distributions are resolved into two components; a velocity components $V_{i l}$ directed along the beam direction, resulting from the initial projectile-target interaction, and a velocity component $V$, isotropic in the moving system, resulting from de-excitation of the moving fragment. These tivo steps are equivalent to the abrasion and ablation steps in the abrasion-ablation model of RHI collisions. The results of this analysis are shown in Fig. III-A-2 values of $\beta_{\|}\left(=V_{\|} / C\right)$ and $\langle T\rangle\left(=\frac{1}{2} A V^{2}\right)$, the average fragment kinetic energy in the system moving with velocity $B_{\|}$are shown as a function of the product mass number $A$. Also shown are data from the interaction of $3 \mathrm{GeV}$ protons with ${ }^{197 \mathrm{Au},}{ }^{7}$ which is taken to represent the maximum momentum transfer to the target residues ever observed in a proton-induced reaction. It appears that the forward velocities, $\beta_{i i}$, of the target fragments from the RHI reaction exceed the corresponding velocities from the proton-induced reaction, especially for the lightest reaction products. While more careful comparisons with data from reactions induced by $1-10 \mathrm{GeV}$ protons are in order, it would seem that one has seen evidence in the target fragment recoil properties for "hard" collisions in RHI induced reactions. Further studies of the target residue kinematic properties in these lower energy RHI-induced reactions should prove to be of importance for our understanding of the mechanism of these reactions.

(W. Loveland, D. J. Morrissey,* K. Aleklett, and G. T. Seaborg*) 


\section{Footnotes and References}

*Permanent address: Lawrence Berkeley Laboratory, Berkeley, CA 94720.

+Permanent address: Studsvik Scientific Research Laboratory, Nyköping, Siveden.

1. D. J. Morrissey, W. Loveland, and G. T. Seaborg, Z. Physik, A289, 123 (1978).

2. J. B. Cumming, R. W. Stoenner, and P. E. Haustein. Phys. Rev. C14, 1554 (1976).

3. S. B. Kaufman, E. P. Steinberg, and. B. D. Hilkins, Phys. Rev, Lett. 41 , $1359(1979)$.

4. S. B. Kaufman, private communication.

5. B. Neidhart and K. Bächmann, J. Inorg. Nuci. Chem. 34, 423 (1972); U. Trabitzsch and K. Bächmann, Radiochimica Acta, 16, 129 (1971).

6. L. Winsberg, Nuc7. Inst. Meth. 150,465 (1978).

7. S. B. Kaufman, E. P. Steinberg, and M. W. Weisfield, Phys. Rev. C18, 1349 (1978). 
Figure III-A-i. Product recoil properties for the reaction of $8 \mathrm{GeV}{ }^{20} \mathrm{Ne}+\mathrm{Ta} . \cdots$ Also shown is the data of reference 5 . 


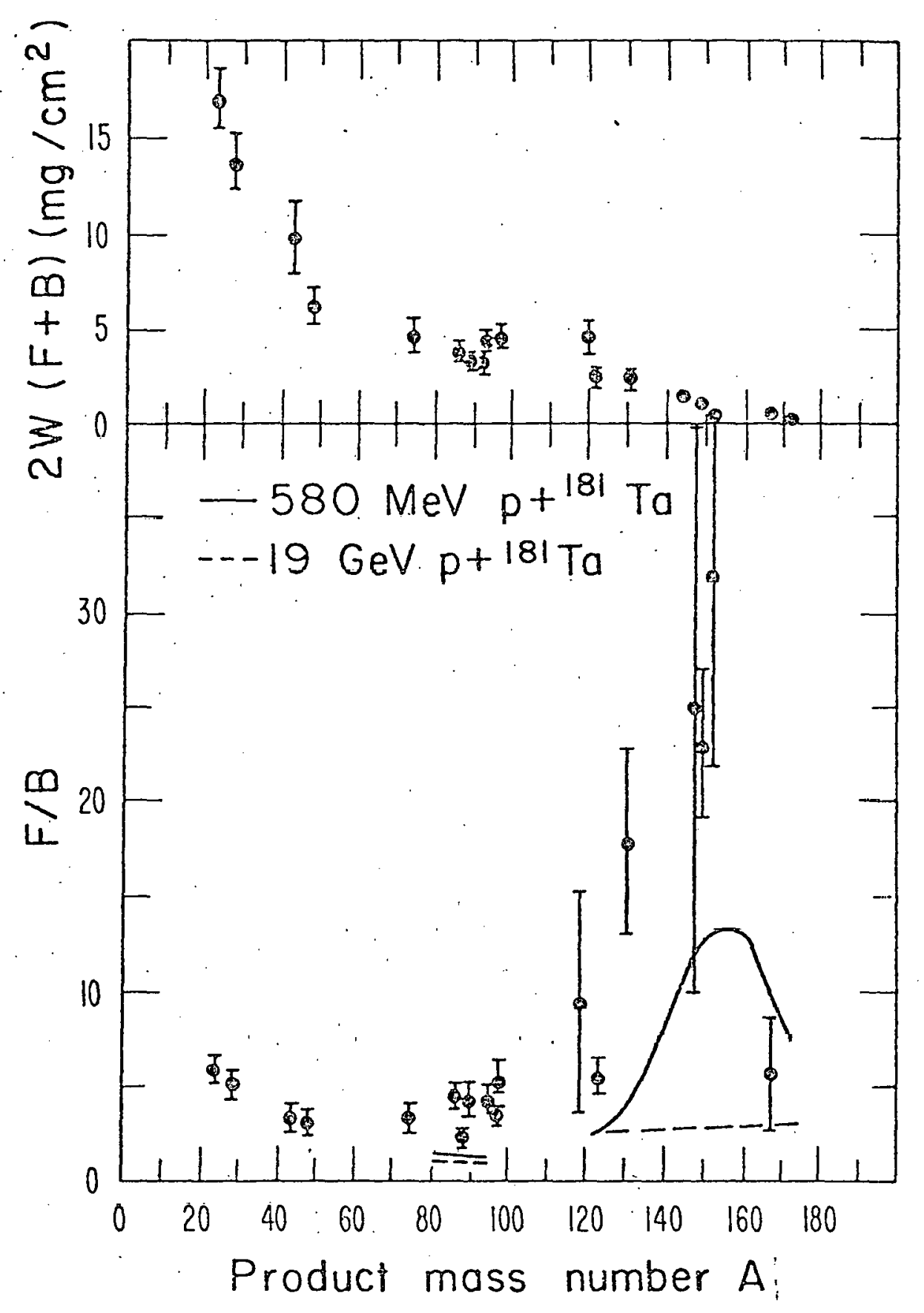

XBL 798-2411 
Figure III-A-2 Product velocity component, $B \|\left(=V_{\|} / c\right)$, resulting from initial projectile-target interaction and average kinetic energy, $\langle T\rangle$, in system moving with velocity $B$ il vs product mass member $A$, in the reaction of $8 \mathrm{GeV} 20 \mathrm{Ne}+\mathrm{Ta}$. 


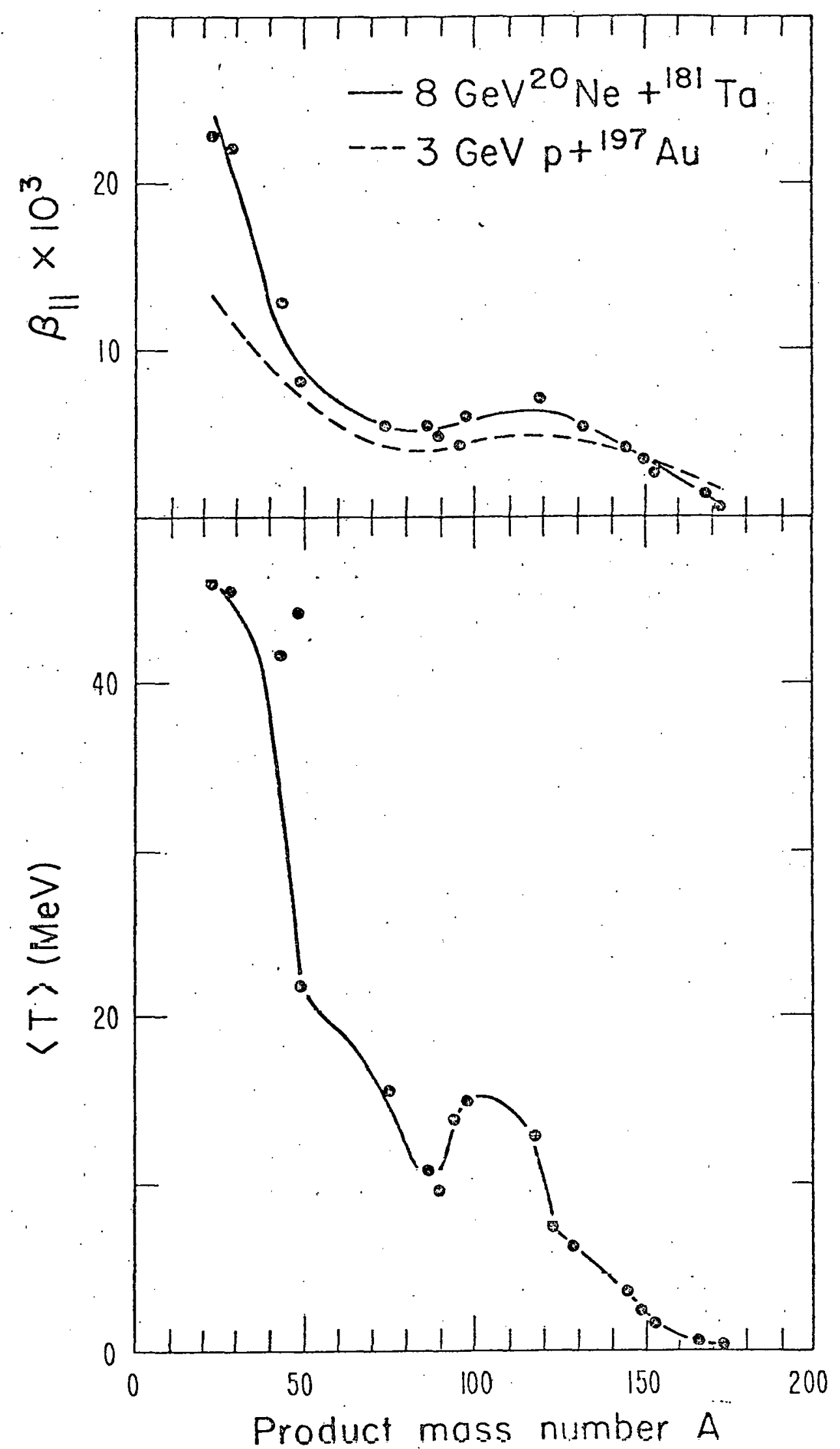

XBL $798-2412$ 
III-B- Measurement of Absolute Cross Sections for the Interaction of $z_{-52}$ GeV ${ }^{12} \mathrm{C}$ $+\frac{U}{3}$

Loveland et al. 1,2 have reported the results of measurements of the product mass and charge distributions in the interaction of $25.2 \mathrm{GeV}{ }^{12} \mathrm{C}$ with targets of $\mathrm{Au}, \mathrm{Pb}, \mathrm{Bi}$, and $\mathrm{U}$. The most striking new feature observed in these studies was the survival of large numbers of heavy products $(150 \leq A \leq 200)$. In the case of the $C+U$ reaction these nuclei were presumably the survivors of interactions involving significant projectile-target overlap. Unfortunately, due to dificulicies in operation of the beam monitoring equipment during the original experiment, only relative production cross sections for these heavy products were measured.

During the past year, we have completed a repetition of the measurement of the product mass and charge distribution for the interaction of $25 \mathrm{GeV}^{12} \mathrm{C}$ with $U$. The results of combining this measurement with the previous one showing absolute product yields are seen in Figure III-B-T along with similar measurements for interaction of $8 \mathrm{GeV}{ }^{20} \mathrm{Ne}$ and $28 \mathrm{GeV} \mathrm{P}$ with $U$. The fission cross section is 01800 $\mathrm{mb}$ with the average fissioning system mass being 2208 for the reaction of $25 \mathrm{GeV}$ ${ }^{12} \mathrm{C}+U$. The cross section for the production of heavy collision residues, $\sigma_{H R}$ is $27300 \mathrm{mb}$. Thus over $85 \%$ of the total reaction cross section, $\sigma_{R_{2}}$ is associated with collisions giving rise to large radioactive target fragments and a correspondingly small fraction of the cross section is associated with catastrophic central collisions with high charged particle multiplicity. A more detailed discussion of these results and a comparison to other systems is found in Section III-C of this report.

(W. Loveland, R. J. Otto, D. J. Morrissey, and G. T. Seaborg)

\section{REFERENCES}

1. W. Loveland, R. J. Otto, D. J. Morrissey, and G. T. Seaborg, "Large Collision Residues and Nuclear Fission in the Interaction of $25.2 \mathrm{GeV}{ }^{12} \mathrm{C}$ with Uranilum," Phys. Rev. Lett. 39, 230 (1977).

2. W. Loveland, R. J. Otto, D. J. Morrissey, and G. T. Seaborg, "Further Studies of Large Collision Residues in Relativistic Heavy Ion Reactions with Heavy Nuciei," Phys. Lett. 69B, 284 (1977). 
Figure III-B-1. Product mass distributions for the reaction of $8 \mathrm{GeV}{ }^{20} \mathrm{Ne}$, $25 \mathrm{GeV}{ }^{12} \mathrm{C}$ and $28 \mathrm{GeV} p$ with $\mathrm{U}$. 


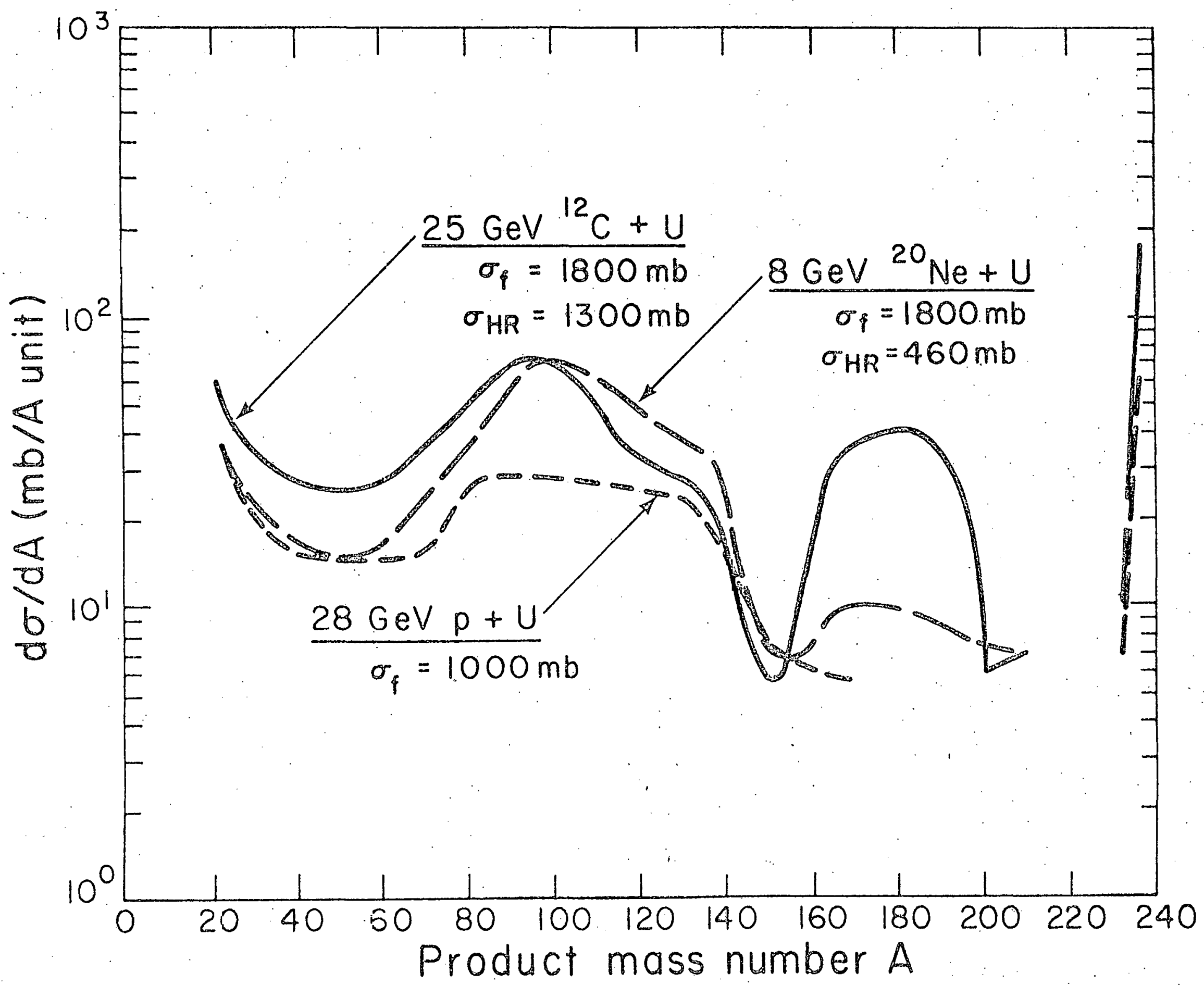


III-C- Radiochemical Studies of Product Mass and Charge Distributions in the Interaction of Relativistic Heavy Ions with Uranium

In the interaction of $25 \mathrm{GeV}{ }^{12} \mathrm{C}$ ions with ${ }^{238} \mathrm{U}$, Loveland et al ${ }^{1}$ reported the occurence of unusually high yields of the lightest products $(A<40)$ and the heavy target residues $(A=140-200)$ when compared to the reaction 4 of $28 \mathrm{GeV}$ protons with ${ }^{238} \mathrm{U}$. We have measured the product mass and charge distribution from the interaction of $8 \mathrm{GeV}{ }^{20} \mathrm{Ne}$ with ${ }^{238} \mathrm{ll}$ to investigate the effect of varying the projectile energy and $(Z, A)$ upon these distributions.

Targets of natural uranium metal varying in thickness from 25 to $75 \mathrm{mg} / \mathrm{cm}^{2}$ and surrounded by $275 \mathrm{mg} / \mathrm{cm}^{2}$ mylar catcher foils were irradiated with $210^{13}$ $8 \mathrm{GeV}{ }^{20} \mathrm{Ne}$ ions at the LBL Bevalac. Immediately following the end of bombardment, gamma-ray spectroscopic measurements of the radioactivities induced in the target and catcher foils were begun using. a coaxial $\mathrm{Ge}(\mathrm{Li})$ detector, and were continued for one month. In order to permit the detection of low level activities normally obscured by high level fission products, one of the uraniurn foils was dissolved and chemically separated into several fractions, ${ }^{2}$ which were then assayed individually.

On the basis of the measured gamma-ray energies, half-lives and relative $\gamma$-ray abundances, over 100 radionuclides were identified. ${ }^{3}$ Each measured activity was corrected for the effects of secondary-induced reactions ( $210-30 \%$ ) based on the variation of the activity with target thickness. The magnitude of the correction factor was found to be linear with target thickness to within experimental error. Independent and isobaric yields were calculated for each observed radionuclide by an iterative fitting and integration procedure, based upon the assumption of a Gaussian charge distribution of the form

$$
P(Z, A)=\left[\left(2 M v^{2}\right)-1 / 2 \exp \left((Z-Z p)^{2} / 2 \sigma^{2}\right)\right] P(A) .
$$

where $P(Z, A)$ is the relative probability for the formation of a nucleus of mass number $A$ and atomic number $Z, P(A)$ is the isobaric yield, and $\sigma$ is the parameter governing width of the distribution in $Z$ units. 
Two separate irradiations of ${ }^{238} \mathrm{U}$ with $8 \mathrm{GeV}{ }^{20} \mathrm{Ne}$ were made and the results were combined to form a single data set. The results of measurements of the product mass and charge distributions from the interaction of $25 \mathrm{GeV}{ }^{12} \mathrm{C}$ with ${ }^{238} \mathrm{U}$ (see Section III-B) were combined with the results of reference 1 to form another data set. The product mass distributions for these two systems are shown in Figure III-C- 1 along with the product mass distribution from the interaction of $28 \mathrm{GeV}$ protons ${ }^{4}$ with $U$. The product $Z$ distributions for constant $A=45,80,100,185$ and the iodine product isotopic distributions are shown in Figures III-C-2 through III-C-6. For the $Z$ distributions at constant $A$, the data points displayed represent yields at the displayed $A$ value and adjacent $A$ values corrected to the displayed A value.

From examining these data, one concludes that fission of $U$ induced by relativistic protons or heavy ions is a similar process. The fission product mass distributions are similar in magnitude or scale (within experimental error) with the total reaction cross section. The mean mass of the fissioning system is estimated from the mass distributions to be essentially the same for proton or heavy ion induced reactions $\left(\bar{A}_{f p} \sim 206\right.$; AfHI $\left.\sim 208\right)$. The fission cross section is estimated to be $07 / 2$ the total reaction croșs section, leading to the estimate that fission events arise from impact parameters, $b, \geq 0.7\left(R_{t}+R_{p}\right)$. The charge dispersions are relatively similar in shape, except perhaps for the $A=100$ fragments where there appears to be some double humped character to the charge distribution from the $\mathrm{Ne}+\mathrm{U}$ reaction. The iodine isotopic distribution shows that as we go from $8 \mathrm{GeV}$ to $25 \mathrm{GeV}$ incident projectile energy, there is only a shift of 2 neutrons in the light iodine distribution, a shift of a few tens of MeV in excitation energy. Detailed examination of the charge distribution widths shows fission in these systems to be a relatively low excitation energy process ( $\left.E^{*}<50 \mathrm{MeV}\right)$.

However, there is a dramatic increase in heavy residue yields as the incident projectile energy increases from 8 to $25 \mathrm{GeV}$. A possible explanation for this effect is that the incident projectile energy increases, the projectile-target interaction results in "cleaner cuts" of projectile and target nuclei through each other. (This speculation is supported by the failure of the extreme clean-cut abrasion-ablation model to describe the interaction of $8 \mathrm{GeV}{ }^{20} \mathrm{Ne}$ with Ta, while it succeeds quite well in describing the $25 \mathrm{GeV}{ }^{12} \mathrm{C}+\mathrm{Ta}$ reaction). The products of the "clean-cut" 25 " GeV C + U reaction are then postulated to have less excitation energy, and 
greater survival probabilities than the products of the "sloppy cut" $8 \mathrm{GeV} 20 \mathrm{Ne}$ $+U$ reaction.

(P. McGaughey, W. Loveland, D. J. Morrissey, R. J. Otto, G. T. Seaborg).

\section{REFERENCES}

1. W. Loveland, R. J. Otto, D. J. Morrissey and G. T. Seaborg, Phys. Rev. Lett. 39, 230 (1977).

2. J. Kratz, J. Liljenzin, and G. T. Seaborg, Inorg. Nucl. Chem. Lett. 10, 951 (1974).

3. D. Morrissey, D. Lee, R. Otto, and G. T. Seaborg, Nucl. Inst. Meth. 158, 499 (1979).

4. Y. Chu, E. Franz, G. Friedlander, and P. Karol, Phys. Rev. C4, 2202 (1971). 
Figure III-C-1 Product mass distributions from the reaction of relativistic projectiles with a uranium target. 


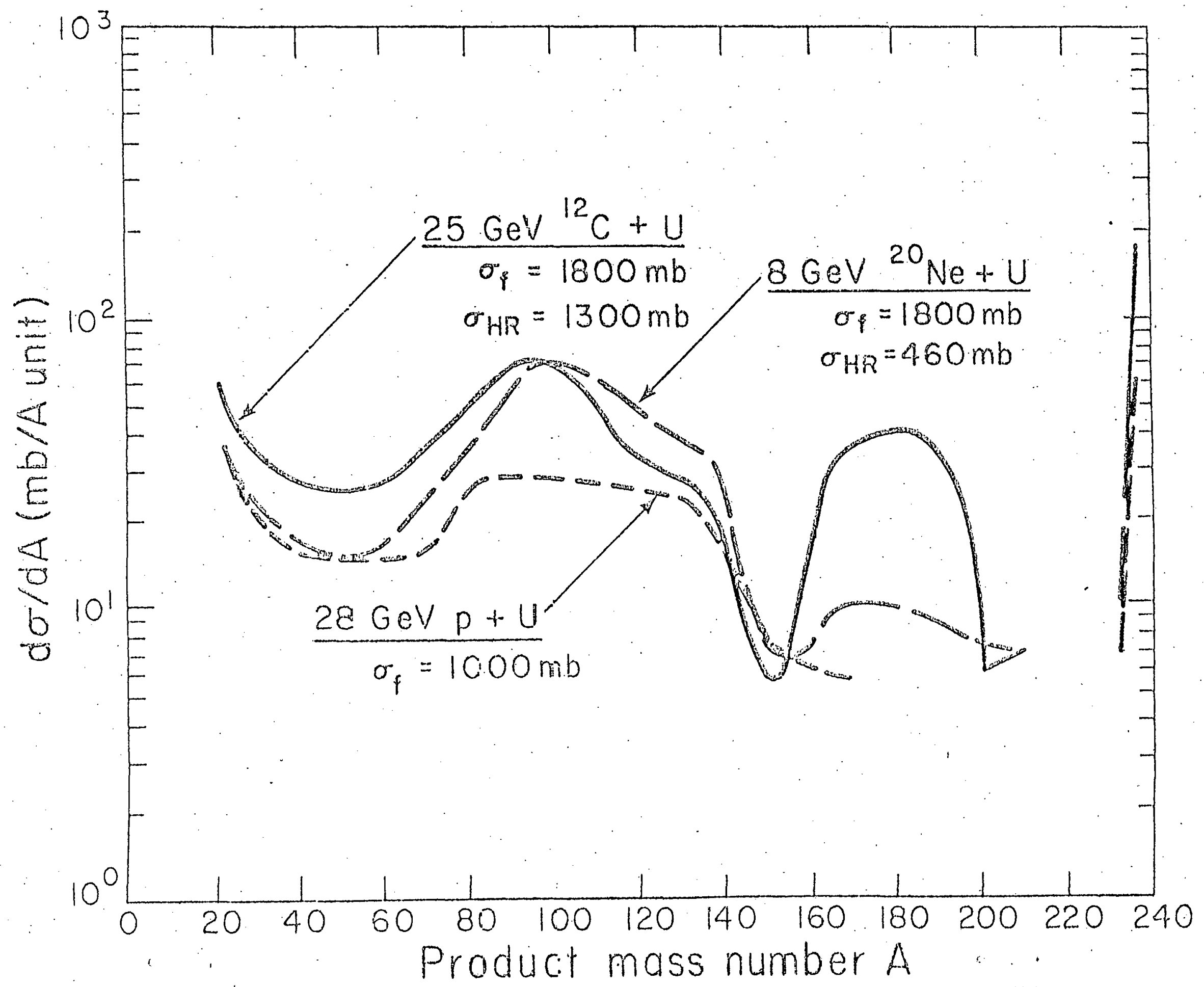


- Figure III-C-2 Indcpendent yield production cross sections for $A=45$ nuclides formed in the interaction of relativistic projectiles with a $U$ target. 


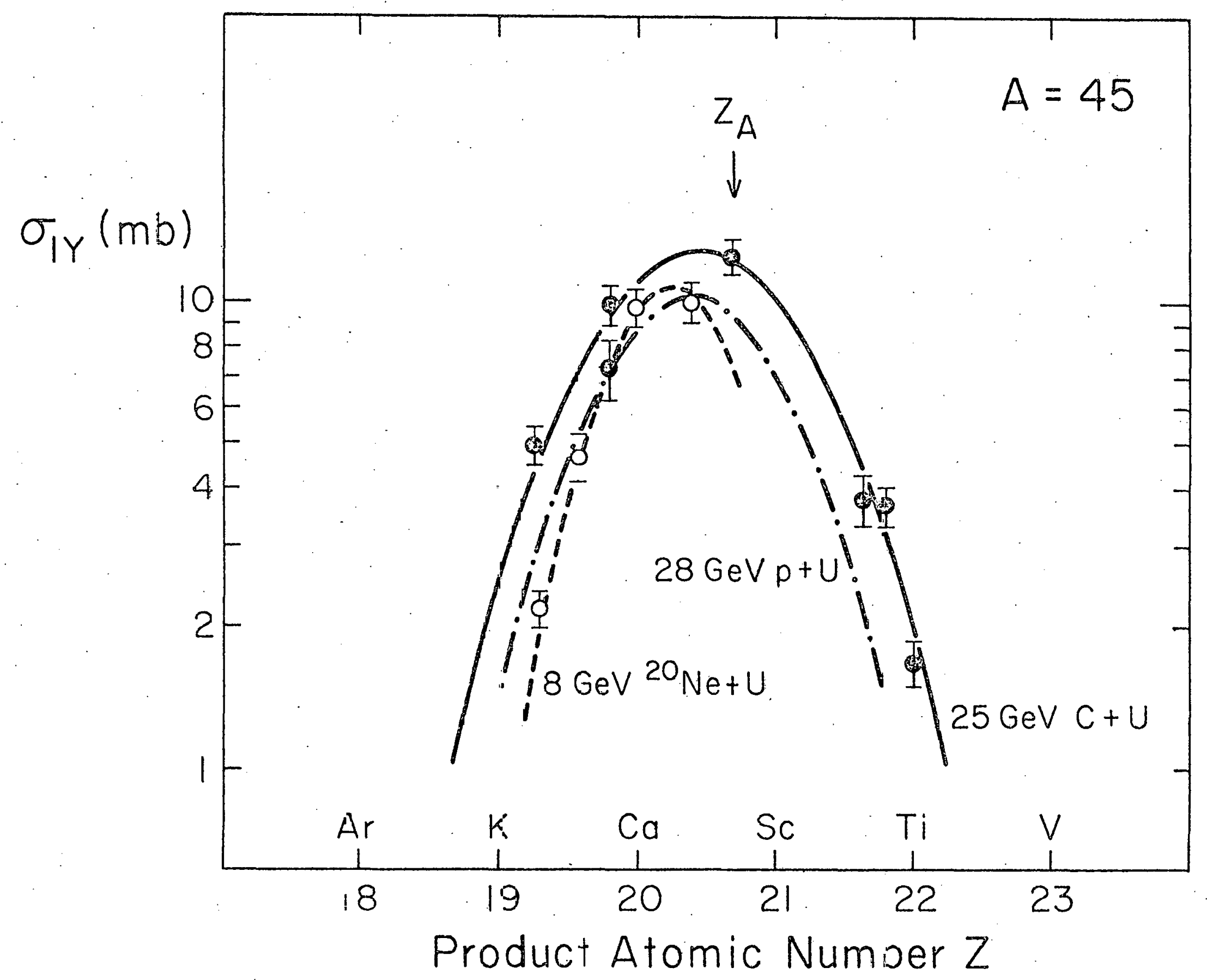

$\omega$ 
Figure III-C-3 Independent yield production cross sections for $A=80$ nuclides formed in the interaction of relativistic projectiles with a uranium target. 


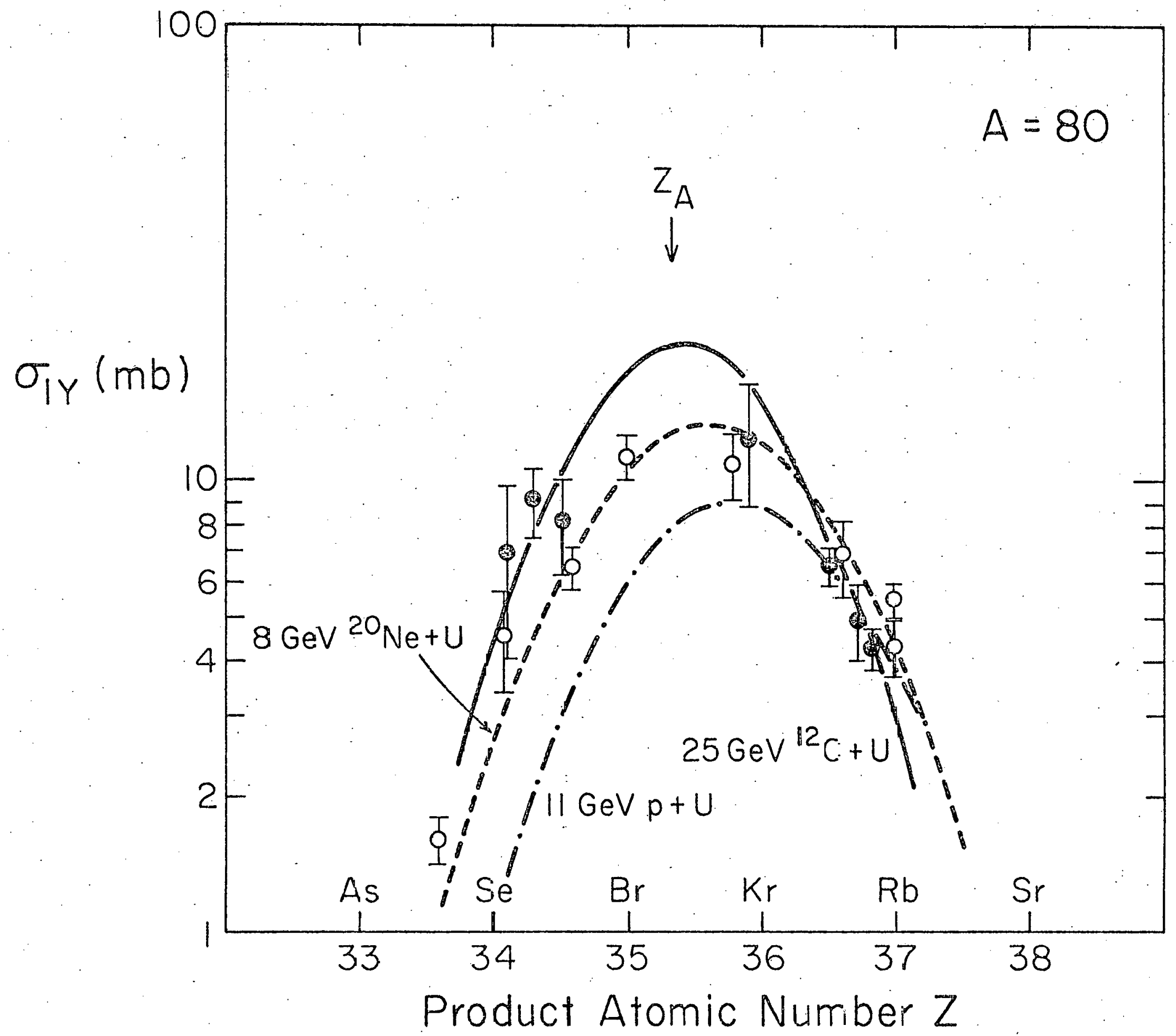


Figure III-C-4 Independent yield production cross sections for $A=100$ nuclides formed in the interaction of relativistic projectiles with a uranium target. 


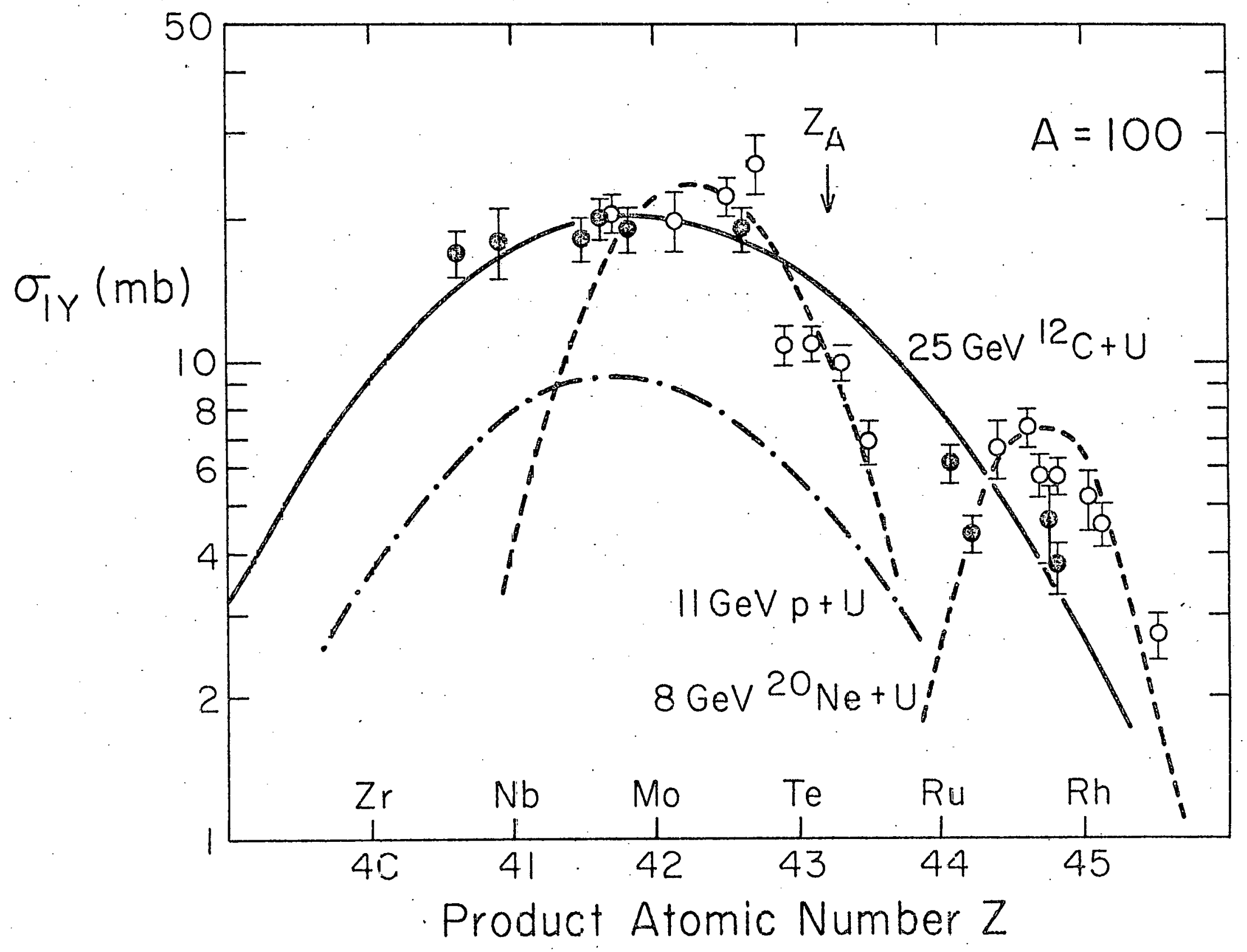


Figure III-C-5 Independent yield production cross sections for $A=185$ nuclides formed in the interaction of relativistic projectiles with a uranium target. 


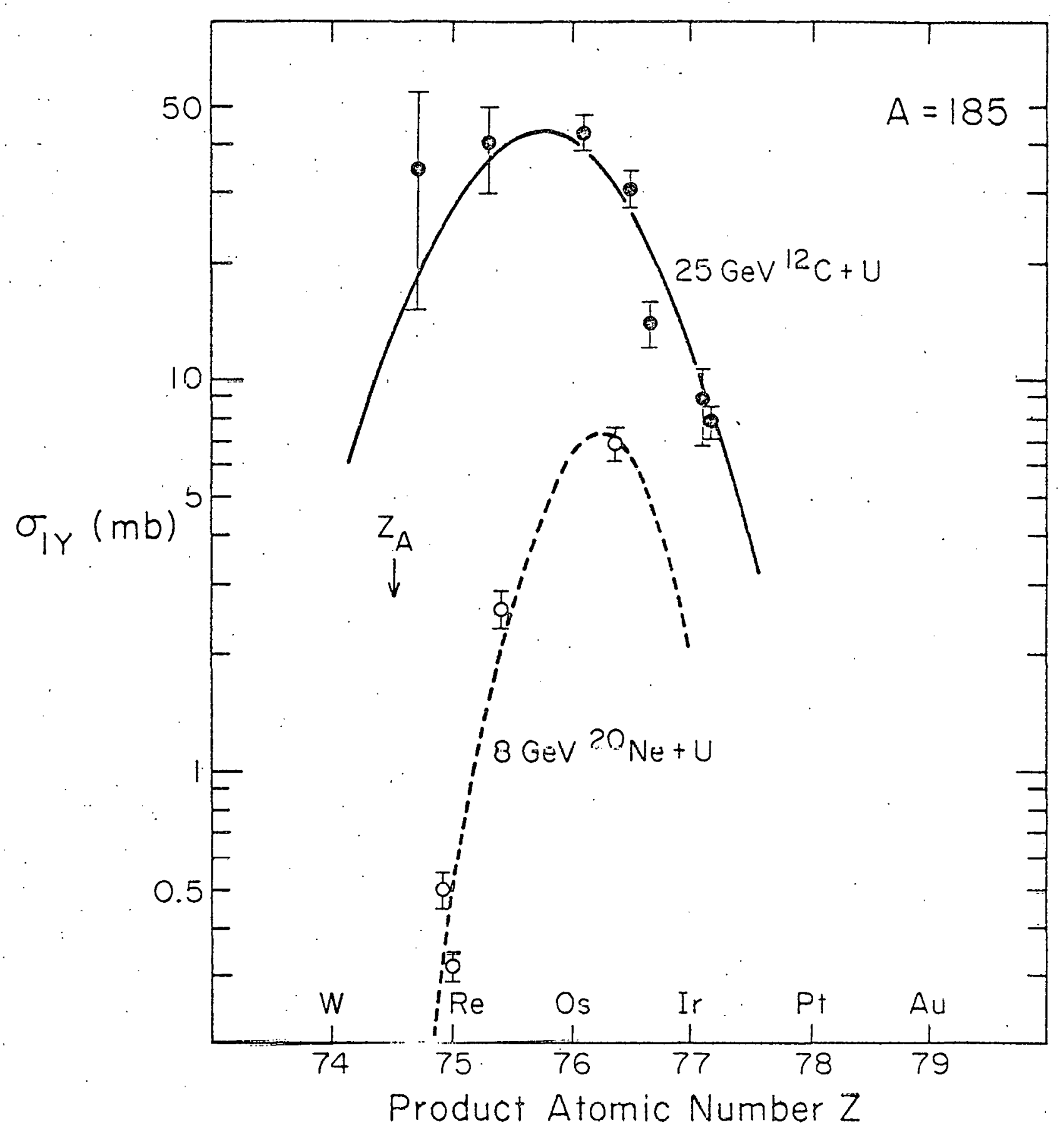


Figure III-C-6. Independent yield production cross sections for $Z=53$ isotopes formed in the interaction of relativistic projectiles with a, uranium target. 


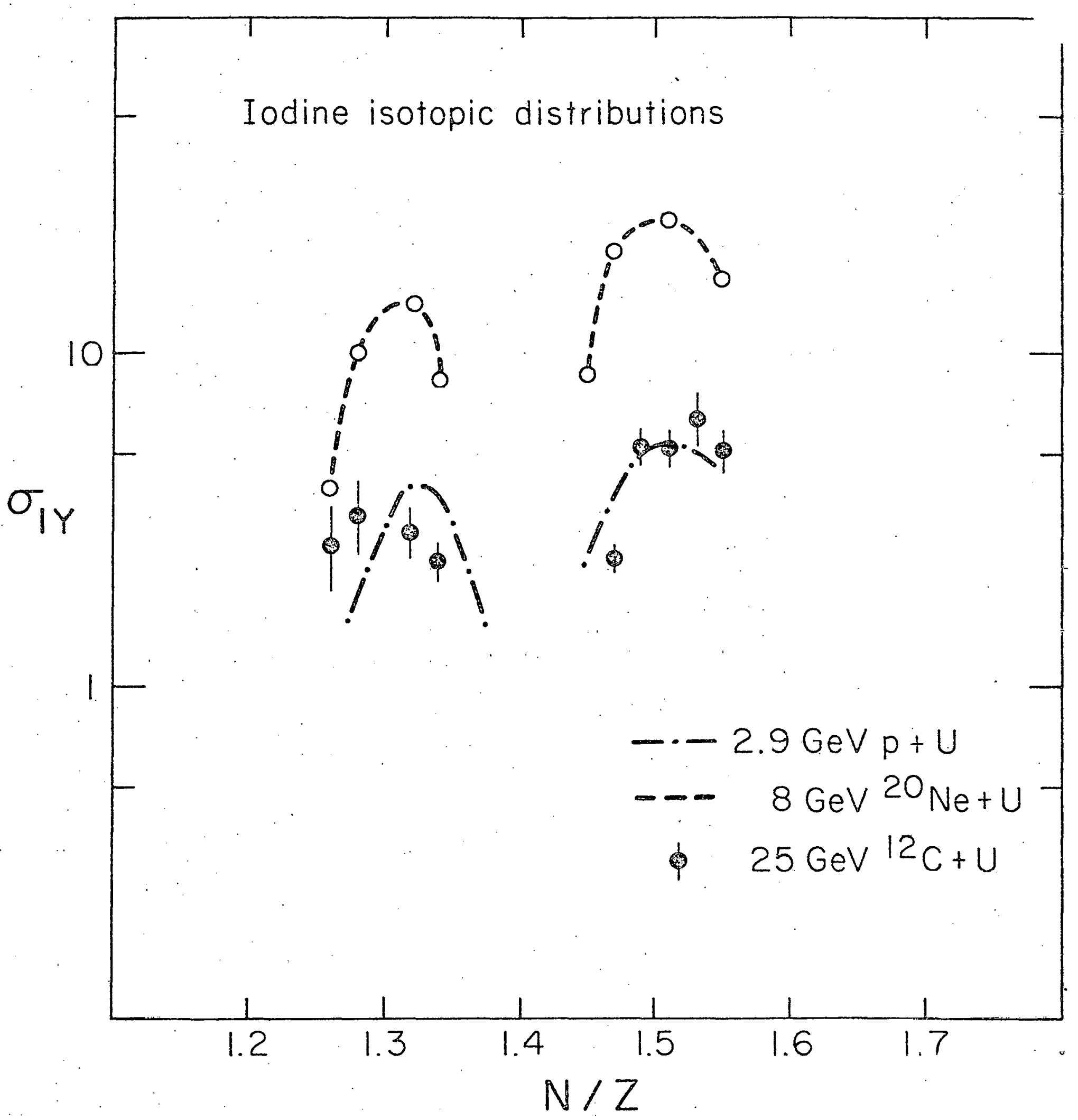


III-D- Target Residue Recoil Properties in the Interaction of Relativistic Heavy Ions with Uranium.

Since the first measurements ${ }^{1}$ of the yields of target fragments from the interaction of relativistic heavy ions (RHI's) with U, it has been apparent that many new and interesting phenomena were to be found. In Sections III-B and III-C of this report, we detail new results on the energy dependence of the large residue yields in these reactions and discuss RHI-induced fission. In Section III-A, we show new insights gained from studying the target fragment recoil properties in a RHI-induced reaction such as the reaction of $8 \mathrm{GeV}{ }^{20} \mathrm{Ne}$ with Ta. With these facts in mind, we began a systematic program of study of the recoil properties of the fragments from the interaction of RHI's with U.

Using the thick target-thick catcher recoil technique, we irradiated depleted $U$ foils (of thickness 56.1 and $51.3 \mathrm{mg} / \mathrm{cm}^{2}$ ) that were surrounded by "forward" and "backward". mylar. catcher foils $\left(235 \mathrm{mg} / \mathrm{cm}^{2}\right)$ with beams of $6.2 \times 10^{13} 4.8 \mathrm{GeV} \cdot{ }^{12} \mathrm{C}$ ions and $1.0 \times 10^{13} 5.0 \mathrm{GeV}{ }^{20} \mathrm{Ne}$ ions, respectively, at the LBL Bevalac. Gamma ray spectrometric measurements of the target fragment radionuclides caught in the forward catcher, backward catcher and target foils were begun after bombardment and continued for 1-2 months. A preliminary analysis of these data has been made and the results are tabulated in Tables III-D-I and III-D-2. In these tables, $F / B$ is the ratio of the number of product atoms recoiling into the forward catcher foil to the number of atoms recoiling into the backward catcher foil, $W$ is the target thickness $\left(\mathrm{mg} / \mathrm{cm}^{2}\right)$ and $(F+B)$ is the fraction of the total number of product atoms recoiling out of the target. The quantity $2 W(F+B)$ is approximately the range of a given product in the target material. Also shown in Tables III-D-I and III-D-2 in parentheses are the values of interaction of approximately equivalent total kinetic energy protons with $U$. Because of the lower particle fluence in the bombardment, the data for the $5.0 \mathrm{GeV}{ }^{20} \mathrm{Ne}+{ }^{238} \mathrm{U}$ system are more uncertain and sparser in character.

From examining the data in these tables, certain general trends bccome apparent. As inferred from the product yields in Section III-C of this report, RHI-induced fission appears to be a process very similar to relativistic proton 
induced fission. The $F / B$ values and approximate ranges of the neutron-rich fission products such as ${ }^{99} \mathrm{Mo}$ or ${ }^{13 \mathrm{I}} \mathrm{I}$, etc. are similar for $\mathrm{C}$, Ne and $p$ induced fission of U. Amongst the lighter "fission" or "deep spallation" products, such as the As isotopes, there appears to be some tendency for longer ranges as one goes to more neutron deficient isotopes. As inferred by Chang and Sugarman ${ }^{2}$ in recoil studies or Wilkins et al. ${ }^{3}$ in counter studies with the interaction of relativistic protons with $U$, this may be evidence for the occurrence of a binary fission process involving low mass nuclei $(A=120-140)$ which are the residues of an intranuclear cascade process involving high deposition energy and the emission of fragments prior to fission both in the fast cascade and the evaporation stage.

Amongst the 7 ightest products from these reactions ( $A<40$ ) the RHI reactions appear to give products of similar range within experimental error but considerably bigger $F / B$ ratios. Interestingly the 7 ight product $F / B$ ratios from the $4.8 \mathrm{GeV}{ }^{12} \mathrm{C}$ ion bombardment $(\nu=20(\mathrm{MeV} / \mathrm{amu}) 1 / 2)$ are substantially shorter than those resulting from the bombardment with ions of approximately the same total kinetic energy projectile $\left(5.0 \mathrm{GeV}{ }^{20} \mathrm{Ne}\right)$ which, of course, has a different velocity, $v \sim 16$ (MeV/amu) $1 / 2$.

The heavy reaction products $(A>140)$ show similar interesting trends. In the reaction of $4.8 \mathrm{GeV}{ }^{12} \mathrm{C}$ with $U$, the ranges of these heavy products appear to be similar to those products produced in relativistic proton-induced reactions but seem to show substantially greater $F / B$ ratios. While the data is quite sparse from the $\mathrm{Ne}$ induced reaction, there appears to be a tendency toward larger $F / B$ ratios, and also longer ranges.

The large F/B ratios associated with the very light and very heavy products may indicate these products result from "hard" nuclear collisions. The short ranges of the heavy products may be due to the notion that they are low excitation energy residues (while their higher excitation pnergy cousins have emitted particles and fissioned giving rise to the n-deficient $A=60-80$ species). A curious aspect of these heavy residues with high $F / B$ ratios is that. they occur in the samn mass range $(A=140-160)$ for three different reactions, $4.8 \mathrm{GeV}{ }^{12} \mathrm{C}+U, 5 \mathrm{GeV}{ }^{20} \mathrm{Ne}+U$, $8 \mathrm{GeV} 2{ }^{\circ} \mathrm{Ne}+\mathrm{Ta}$. While there are other interesting speculations one may make about. this data, it is clear that further, more extensive studies of this type may substantially improve our knowledge about the production of target fragments in relativistic nuclear collisions.

(Luo Cheng, W. Loveland, D. J. Morrissey and G. T. Seaborg) 
Table III-0-1

Target Residue Recoil Properties

$4.8 \mathrm{GeV}{ }^{12} \mathrm{C}+{ }^{239} \mathrm{U}$

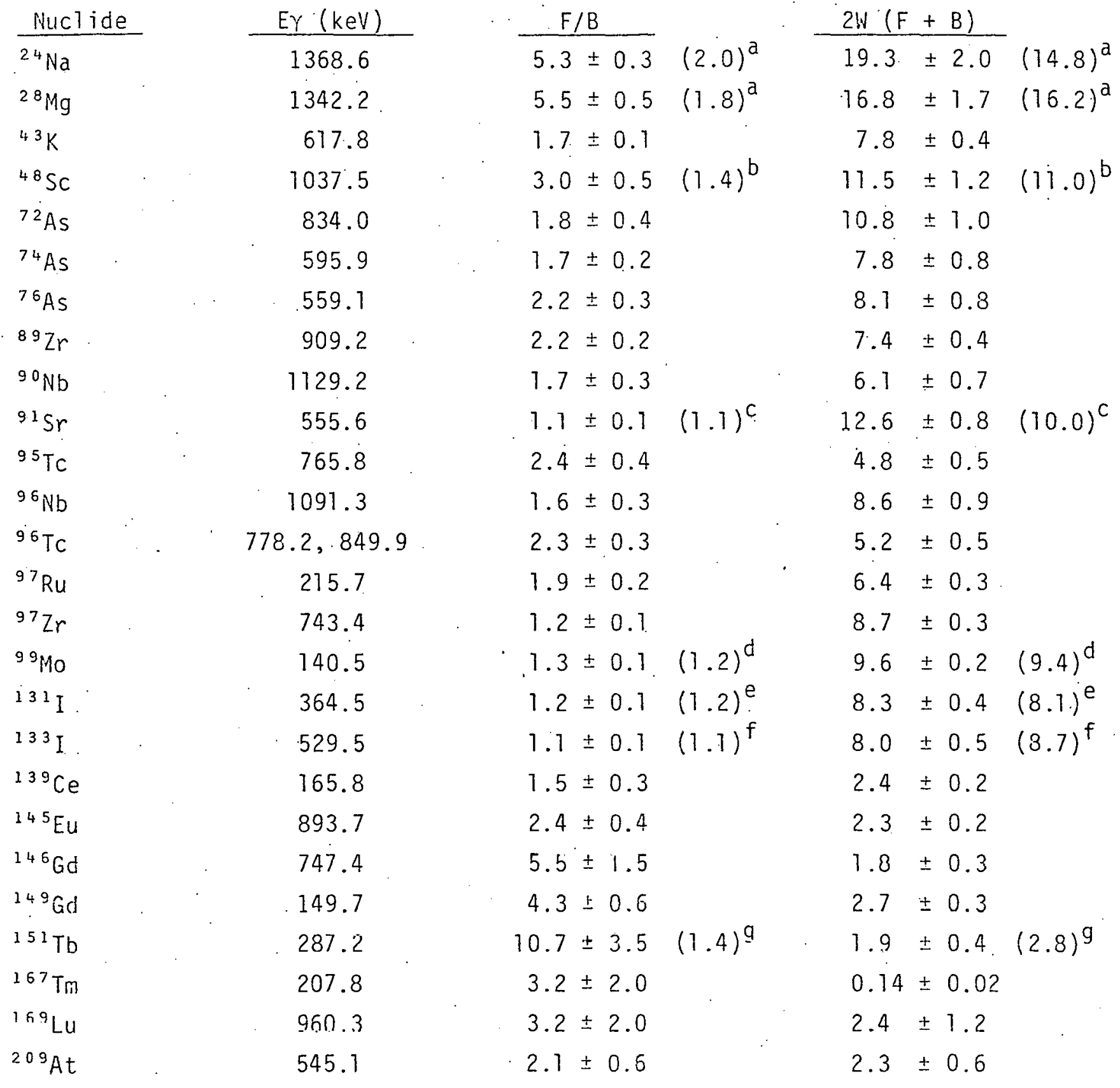

a. S. B. Kaufman and M. W. Weisfield, Phys. Rev. C11, 1258 (1975).

b. 0. Scheidemann and N. T. Porile, Phys. Rey. Cl $\overline{4}, 1534$ (1976).

C. K. T. Beg and N. T. Porile, Phys. Rev. C3, $1 \overline{631}$ (1971).

d. J. M. Alexander, C. Baltzinger and M. F. Gazdik, Phys. Rev. 129, 1826 (1963).

e. S. Biswas and H. T. Porile (to he published).

f. Y. W. Ylu and N. T. Porile, Phys. Rev. C10, 167 (1974).

g. P. M. Starzyk, and N. Sugarman, Phys. Rev. C8, 1448 (1973). 
Table III-D-2

Target Residue Recoil Properties

$$
5.0 \mathrm{GeV}{ }^{20} \mathrm{Ne}+238 \mathrm{U}
$$

Nuctide

$24 \mathrm{Na}$
$28 \mathrm{Mg}$
$43 \mathrm{~K}$
$48 \mathrm{SC}$
${ }^{72} \mathrm{AS}$
${ }^{76} \mathrm{AS}$
${ }^{87 \mathrm{Y}}$
$89 \mathrm{Zr}$
${ }^{96} \mathrm{TC}$
$96 \mathrm{Nb}$
${ }^{37} \mathrm{Zr}$
$99 \mathrm{MO}$
$131 \mathrm{I}$
$133 \mathrm{I}$
$146 \mathrm{Gd}$
$144 \mathrm{Gd}$
$160 \mathrm{Er}$

\begin{tabular}{c} 
Ey (keV) \\
\hline 1368.6 \\
1342.2 \\
617.8 \\
1037.5 \\
834.0 \\
559.1 \\
484.8 \\
909.2 \\
778.2 .849 .9 \\
1091.3 \\
743.4 \\
140.5 \\
364.5 \\
529.5 \\
747.4 \\
149.7 \\
728.1
\end{tabular}

\begin{tabular}{l}
$F / B$ \\
\hline $9.8 \pm 2.0$ \\
$5.6 \pm 1.6$ \\
$1.9 \pm 0.3$ \\
$2.4 \pm 0.8$ \\
$2.6 \pm 0.7$ \\
$6.1 \pm 3.0$ \\
$2.1 \pm 0.4$ \\
$1.4 \pm 0.3$ \\
$2.6 \pm 0.6$ \\
$2.5 \pm 0.9$ \\
$1.3 \pm 0.3$ \\
$1.3 \pm 0.1$ \\
$1.2 \pm 0.1$ \\
$1.3 \pm 0.2$ \\
$8.0 \pm 2.0$ \\
$4.6 \pm 1.5$ \\
$1.7 \pm 1.0$
\end{tabular}

$2 W(F+B)$

$20.5 \pm 4.0$ $24.5 \div 6.0$ $8.1 \pm 0.8$ $10.9 \pm 3.0$ $7.9 \pm 2.4$ $3.7 \pm 1.0$ $7.5 \pm 1.1$ $8.8 \pm 1.8$ $8.0 \pm 1.0$ $8.8 \pm 3.0$ $7.9 \pm 0.4$ $9.5 \pm 0.3$ $6.6 \pm 1.0$ $8.7 \pm 1.2$ $9.7 \pm 3.0$ $3.0 \pm 1.0$ $4.7 \pm 1.5$ 


\section{REFERENCES}

1. W. Loveland, R. J. Otto, D. J. Morrissey, and G. T. Seaborg, Phys. Rev. Lett. $\underline{39}, 320(1977)$.

2. S. K. Chang and N. Sugarman, Phys. Rev. C8, 775 (1973).

3. B. D. Wilkens, et al. (private communication).

- III-E- Near Target Residues from the Peripheral Interaction of Relativistic Heavy Ions with Bismuth

Isotopic distributions for the near target residues $A u$ and $T 1$ have been measured radioanalytically for the reaction of $8.0 \mathrm{GeV}{ }^{20} \mathrm{Ne}$ ions with ${ }^{209} \mathrm{Bi}$. We have also calculated the isotopic production cross section for $A u$ and $T 7$ isotopes using a macroscopic abrasion-ablation model and a microscopic intranuclear cascade-evaporation model. The importance of the neutron skin in determining the yield of these products from the peripheral interactions was also explored in the framework of the macroscopic model.

Two $99.999 \%$ pure $B i$ foils (of thickness 116 and $137 \mathrm{mg} / \mathrm{cm}^{2}$ ) surrounded by $35 \mathrm{mg} / \mathrm{cm}^{2}$ mylar catcher foils, were irradiated at the LBL Bevalac for 18.5 and 21.3 hours, with $3.6410^{13}$ and $3.7510^{13}{ }^{20}$ Ne ions, respectively. To increase the detection sensitivity for the $A u$ and $T l$ isotopes a chemical separation of these activities from the $137 \mathrm{mg} / \mathrm{cm}^{2}$ foil was performed. One $\mathrm{mg}$ each of $\mathrm{Au}$ and $\mathrm{Tl}$ carriers were added and dissolved with the foil in $2 \mathrm{ml}$ conc. $\mathrm{HCl}$ and $4 \mathrm{ml}$ conc. $\mathrm{HNO}_{3}$. After the reaction stopped, the oxides of nitrogen were expelled by boiling. The solution was cooled and about $4 \mathrm{ml} \mathrm{H} \mathrm{H}_{2} \mathrm{O}$ added. Then two extractions with $2 \mathrm{ml}$ ethyl ether were made. The ether fractions, containing Au and $\mathrm{Tl}$, were removed from the top of the aqueous phase and combined in another test tube. This was washed two times with $1 \mathrm{ml} 1.5 \mathrm{M} \mathrm{HCl}$, and finally chemically plated on a Pt disk. The chemical procedure took about 20 minutes.

The gamma activities in the chemical Au/TT fraction and the unseparated foil were measured for 4 and 6 weeks, respectiveiy, using a $16 \% \mathrm{Ge}(\mathrm{Li})$ detector with a. FWHM of $1.9 \mathrm{keV}$ at $1332.5 \mathrm{keV}$. Nine isotopes of each element were identified. By comparing gamma lines from the unseparated foil to those in the chemical fraction it was possible to determine the chemical yield for Au to ba $78 \%$ and for $\mathrm{Tl}$ to be $79 \%$. The production cross sections were calculated from the gamma activities, which were corrected for detector efficiency and abundance,' and extrapolated to the end of the irradiation period. Following correction for growth and decay during the bombardment as well as for the fluctuation of the beam intensity, the cross sections were calculated. The Au and Tl cross sections 
are shown by the solid points in Fig. III-E-1. The neutron deficient parts of the distributions were not possible to detect with the radiochemistry used due to the short half-lives of these isotopes. Also, the absolute intensities of the gamma rays of the very neutron deficient $A u$ isotopes are unknown at present.

In the abrasion-ablation view of the collision of RHI with a target nucleus, the two nuclei are taken to be uniform hard spheres which move on straight line trajectories. 2 Those nucleons that 1 ie in the region of overlap of the two nuclei are sheared off in the abrasion (or fast) stage of the collision. The spectator fragments of the targets, which consist of the nucleons that were outside the reyiun of overlap, are assigned an excitation energy that is proportional to their excess surface area. This is the minimum excitation enerqy that sur.h fragments would be expected to have, and in addition increases due to frictional forces are likely to be present. The centroid of the isotopic distributions is given as a first approximation by the neutron to proton ratio of the target nucleus. The variance of the neutron to proton ratio can be calculated in the statistical limit $^{2}$ or through ground state fluctuations of normal nuclear matter. 3,4 For heavy nuclei, however, there is an excess of neutrons in the peripheral or skin region. Therefore, a larger number of neutrons than protons will be abraded in grazing collisions. The neutron to proton ratio for the abraded nuclear matter from ${ }^{209} \mathrm{Bi}$ bombarded with ${ }^{20} \mathrm{Ne}$ was calculated as a function of the number of removed nucleons using the effective sharp radii for the neutron and proton distributions given by Myers and Swiatecki. ${ }^{5}$ (See Fig. III-E-2). Such an introduction of the neutron skin into the calculation produces a shift of the centroid of the primary target fragment distribution for Au and T1 isotopes of about 1.5 mass units to the neutron deficient side.

In the microscopic approach the collision of the RHI projectile with the target nucleus is treated as a two-step process in a Monte Carlo cascade calculation. A fast step occurs with cascading collisinns of nucleons from one reaction partner inside the nucleus of the other partner, which is followed by a slow statistical evaporation of the primary fragments after the fast cascading nucleons have escaped or have been captured by the primary fragments. The calculation is made using an extension ${ }^{6}$ of the intranuclear cascade code, V[GAS, for proton induced reactions which has been modified to treat two colliding nuclei. The two nuclei have diffuse nuclear density distributions, and consideration of 
the Fermi motion of the nucleons is also included. The neutron or proton nature of the collision partners is selected at random in proportion to their number in the nucleus. The impact parameter is also selected at random, and the final cross sections are integrated over the range of impact parameters. The primary fragments are subsequently individually de-excited using a version of the Dostrovsky, Frankel, and Friedlander statistical model Monte Carlo calculations. ${ }^{8,6}$ The excitation energy of each fragment was obtained from the fast cascade code.

In Fig. III-E-3 we present the comparison of the calculations of the final product cross sections with the experimental results. The correlated abrasionablation results are given as the solid line for calculations with uniform nuclei and as the dotted line for the calculations with the neutron skin. The uncorrelated cascade evaporation results are given as histograms. Large differences are immediately apparent between the two types of calculations. The uncorrelated final product distribution is approximately three times as wide as the correlated one, and although flatter, contains slightly more cross section. When compared with the experimental cross sections the abrasion-ablation calculation predicts nuclei which are too neutron excessive. This may be due to an underestimation of the excitation of the fragments. One also sees an odd-even effect that is bigger than experimentally observed. The uncorrelated cascade-evaporation calculations reproduce the experimental cross sections well in the mass region covered. To get a better picture of the neutron deficient part of the distribution, a mass region with long-lived neutron deficient isotopes should be studied or an on-line kinetic method has to be applied. These possibilities are being explored but may have to wait for the acceleration of heavy beams in the upgraded Bevalac allowing these products to be produced as projectile fragments.

(K. Aleklett, D. J. Morrissey, W. Loveland, K. Moody and G. T. Seaborg)

\section{REFERENCES}

1. U. Keus, W. Westmeier, and I. Warnecke, Gesellschaft fur Schwerionenforschung, Report. GSI 79-2.

2. Seefor example, L. F. Oliveira, R. Donange10, and J.0. Rasmussen, Phys. Rev. Cl9, 826 (1979), and references therein.

3. D. J. Morrissey, W. R. Marsh, R. J. Otto, H. Loveland, and G. T. Seaborg, Phys. Rev. $\underline{\text { C18 }}, 1267$ (1978). 
Figure III-E-1 Experimental cross sections for $A u$ and $T I$ isotopes are given as the solid points and the solid curves are Gaussian functions drawn through the data points by eye. XBL 798-2428. 
Figure III-E-2 The neutron to proton ratio of the abraded nuclear matter from ${ }^{209} \mathrm{Bi}$ bombarded with ${ }^{20} \mathrm{Ne}$, as a function of the number of removed nucleons, calculated using the effective sharp radii for the neutron and proton distributions given in Ref. 5 is shown by the solid line. The broken line is the same ratio salculated with a uniform nucleus. XBL 798-2429. 


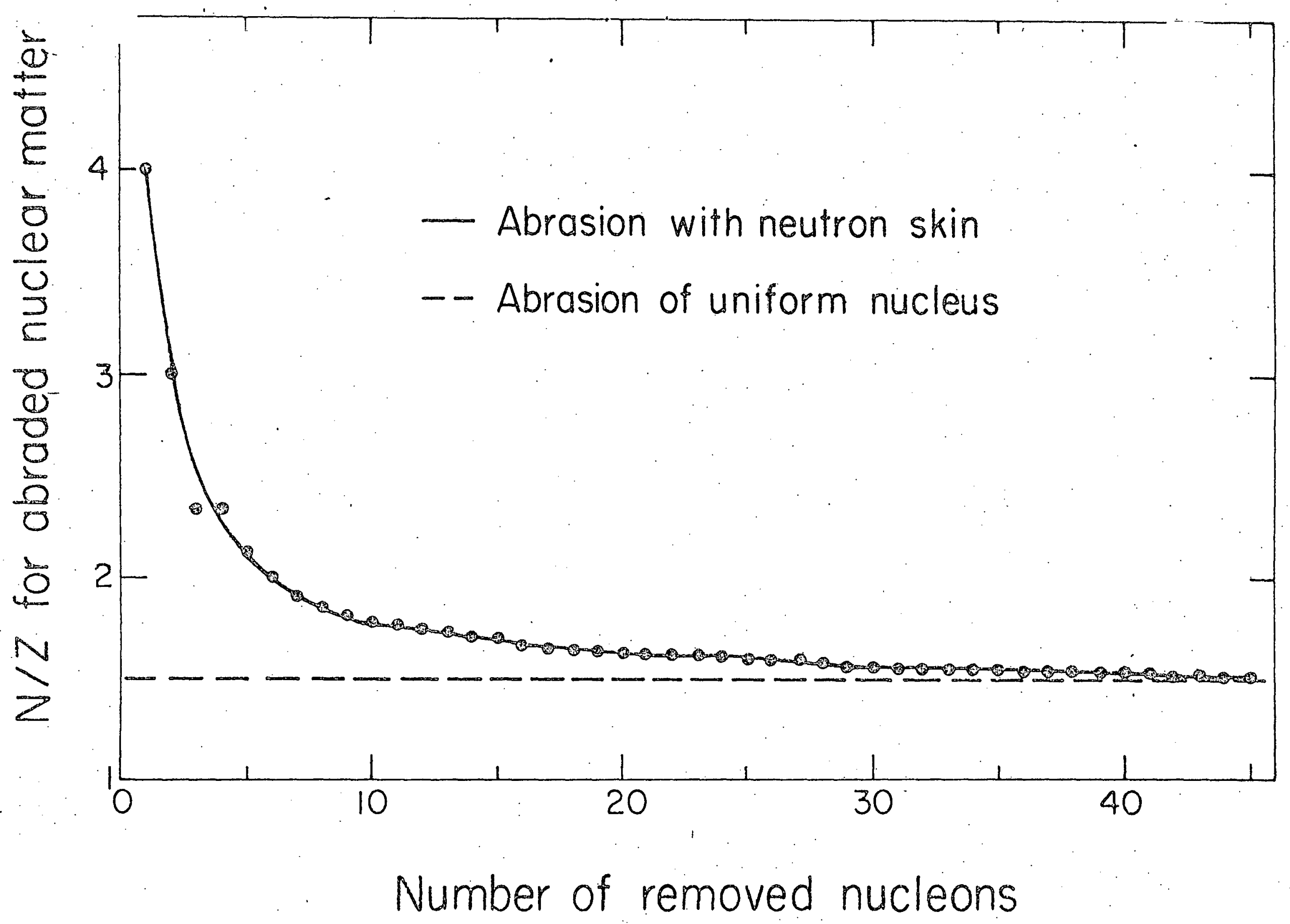

$\stackrel{N}{N}$ 
Figure III-E-3 The calculated values of the production cross sections for Au and $T l$ isotopes are shown for the cascade evaporation calculation (histograms), for the abrasion-ablation with uniform nuclei (solid line), and with the neutron skin (broken line). The data "from Fig. 1 are shown as the solid points. XBL 798-2430. 


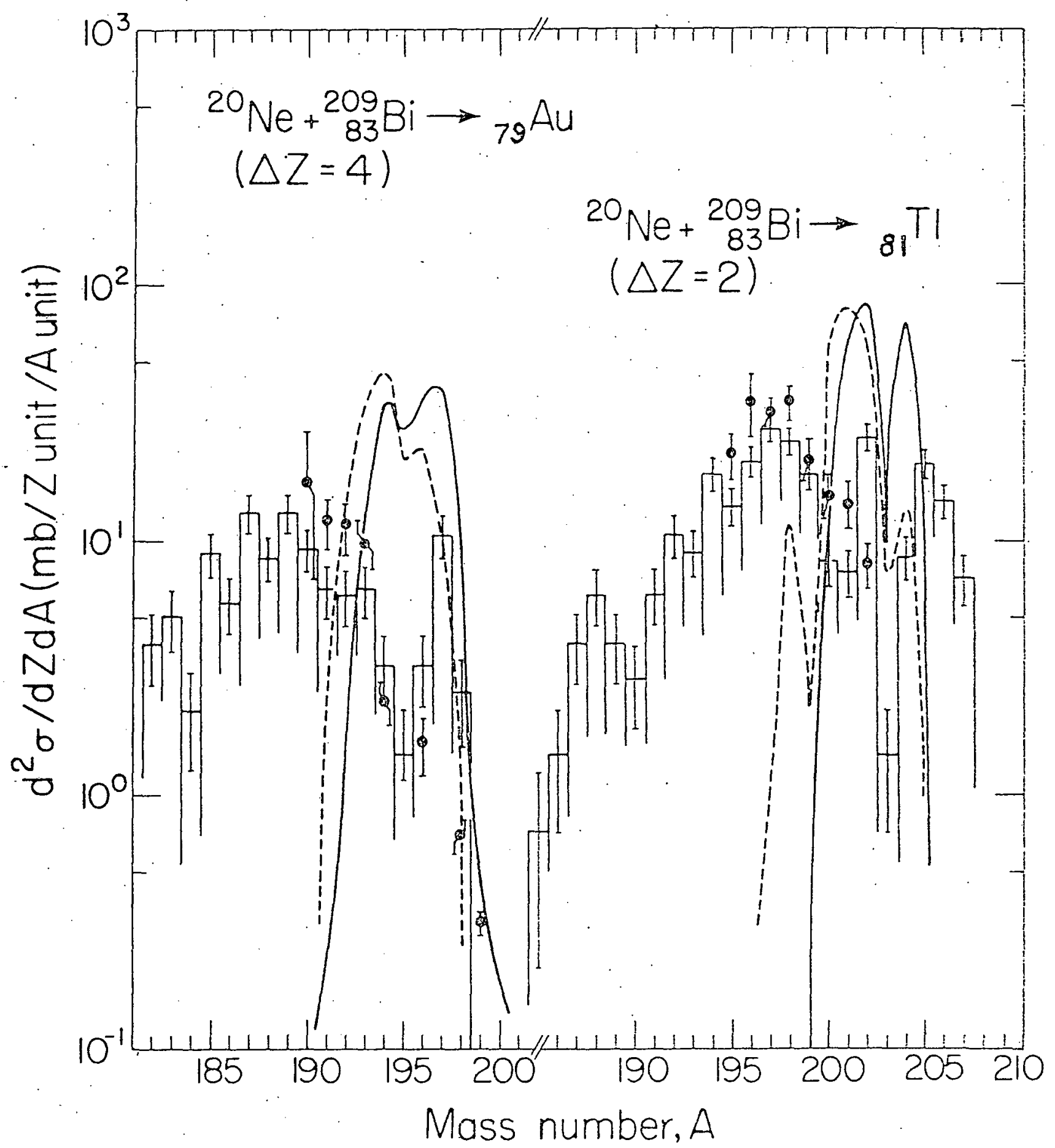

XBL $799-2430$ 
4. J. P. Bondorf, G. Fai, and 0. B. Nielson, Phys. Rev. Lett. 41, 391 (1978); Nucl. Phys. A312, 149 (1978).

5. W. D. Myers and W. J. Swiatecki, Lawrence Berkeley Laboratory Report LBL-9306, 1979.

6. Y. Yariv and $Z$. Fraenkel, to be published.

7. K. Chen, Z. Fraenkel, G. Friedlander, J. R. Grover, J. M. Miller, and Y. Shimamoto, Phys. Rev. 166, 949 (1968).

8. I. Dostrovsky, Z. Fraenkel, and G. Friedlander, Phys. Rev. 116, 683 (1959).

III. F. - Target Residues from the Reaction of $8 \mathrm{GeV}{ }^{20} \mathrm{Ne}$ with $181 \mathrm{Ta}$ and $197 \mathrm{Au}^{*}$

In this paper, we report the study of the reaction of $400 \mathrm{MeV} / \mathrm{A}{ }^{20} \mathrm{Ne}$ projectiles $(8.0 \mathrm{GeV}$ total energy) with $181 \mathrm{Ta}$ and $197 \mathrm{Au}$ targets. The choice of this projectile and energy allows us to make comparisons with the earlier studies of the proton induced reactions and also allows us to study the interaction of RHI at an energy lower than the previously reported studies.

As in previously reported radioanalytical measurements of the target residue products from RHI reactions, these products are observed through gamma-ray spectroscopy of the target materials subsequent to the irradiation. The ${ }^{197} \mathrm{Au}$ targets consisted of two foils, $125 \mathrm{rm}$ by $10.2 \mathrm{~cm}$ each, with thicknesses $49.3 \mathrm{mg} / \mathrm{cm}^{2}$ and $242.0 \mathrm{mg} / \mathrm{cm}^{2}$, each surrounded by $a .6 .6 \mathrm{mg} / \mathrm{cm}^{2} \mathrm{Al}$ catcher foils. These two targets, differing by a factor of approximately 5 in thickness, allowed us to evaluate the contribution from secondary particle induced reactions to the RHI reaction products. The 181 T'a target consisted of a single foil, $13.6 \mathrm{~cm}$ by $10.2 \mathrm{~cm}$, of thickness $154.1 \mathrm{mg} / \mathrm{cm}^{2}$, also surrounded by $6.6 \mathrm{mg} / \mathrm{cm}^{2}$, Al foils: An external beam of $20 \mathrm{Ne}$ was delivered to a $21 \mathrm{~m}$ aị gap by the Bevalac at the Layrence Berkeley Laboratory. Two separate irradiations were performed, one of the gold fojls and one of the tantalum foil.

Gamma-ray spectrometric measurements were made with a single Ge(Li) detector on $25-10 \mathrm{~cm}^{2}$ area pieces cut from the irradiated target foils, which were centered on the beam spot. These measurements began $\sim 20$ minutes after the end of bombardment (EOB) for the gold targets, 274 hours after EOB for the tantalum target, and continued essentially uninterrupted for $\sim 40$ days. The identification of the product radioactivities and the calculation of their production cross sections has been described in detail elsewhere. ${ }^{2}$ Seventy-seven different radioactivities were observed in the two gold targets $(36$ in the thinner target and 66 in the thicker target which includes those observed in both) and 74 radioactivities were identified as reaction products in the tantalum target. In a 11 , 109 different radioactivities wore identified. 
The radiochemical cross sections, whose measurement was described above, represent post neutron and charged particle evaporation, post "fast" beta decay production cross sections. Thus, in order to obtain true production cross sections one needs to correct radiochemically measured cross sections for beta decay, when possible, that occurs between the time of their production in the nuclear reaction and the time at which they are delected through their own beta decay. Once corrected these values of the independent production cross sections, $d^{2} \sigma / d Z d A$, can be used to calculate the mass or charge yield, $d_{\sigma} / d A$ or $d_{\sigma} / d z$ respectively, and also to estimate the total cross section for target residue production.

Comparison of the mass distribution from the reaction of $8.0 \mathrm{GeV} 20 \mathrm{Ne}$ with $181 \mathrm{Ta}$ with the previous studies of proton induced reactions, 3,4 seen in Fig. III-F-1, shows the striking agreement of the RHI results with those from proton induced reactions of the (approximately) equivalent total projectile energy. This agreement is confirmed by the comparison of the $20 \mathrm{Ne}+197 \mathrm{Au}$ mass distribution with the result.s of Kaufman et al. ${ }^{5}$ for the reaction of $11.5 \mathrm{GeV}$

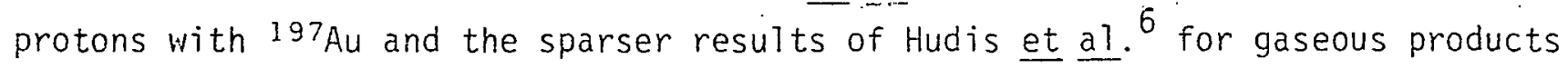
from the reaction of $29 \mathrm{GeV}$ protons with ${ }^{197} \mathrm{Au}$. This comparison can be seen in Fig. III-F-2.

Integration of the mass yield curves, $d s / d A$, over mass number gives the cross section for the production of target residues. We have chosen to integrate these curves over the interval from mass number 40 to the mass of the target. We have chosen a lower limit of 40 mass units for several reasons, (a) the multiplicity of fragments with masses smal.7er than $\sim 40$ is unknown, that is, these products may arise from interactions where another heavy fragment also survives. This certainly is true for fragments such as protons and $4 \mathrm{He}$, where the number of such fragments per event is much greater than one. And (b), because in studies such as this one very little data exists for these low mass products.

The results of this integration are contained in Table III-F-I. The total cross sections for the production of heavy target residues from 131Ta and 197Au were found ton he $2.8 \pm 0.5$ barns. The fact that a small difference that would be expected on the basis of mass number difference was not seen between the two targets is due to the scatter in the calculated mass yields. For comparison we have included two calculations of the total reaction cross section in Table III-F-I. The hard sphere calculation refers to the overlap form of the sharp sphere model where the total reaction cross section is written:

$$
\sigma_{R}=\pi r_{0}^{2}\left(A_{T}^{1 / 3}+A_{p}^{1 / 3}-b_{T_{p}}\right)^{2}
$$


We have taken the parameters $r_{0}$ and $b_{T}$ to be $1.37 \mathrm{fm}$ and $0.57 \mathrm{fm}$. respectively, from the work of Heckman et al. on projectile fragmentation. The soft sphere calculations are those of Karol which were developed to take into account both the diffuse nuclear surface and the variation of the nucleon-nucleon cross section with energy. 8 The comparison of the hard sphere calculations with our measured heavy fragment cross sections show that approximately 75 percent of the reaction cross section gives rise to these products. The soft sphere calculation would indicate that these products represent approximately 80 percent of the reaction cross section. An estimate of the impact parameter range that gives rise to these products can be made from the fraction of the total reaction cross section that they represent. Thus, 75 percent of the hard sphere reaction cross section would lie between the impact. parameter range of $0.5\left(R_{T}+R_{p}\right) \leq b \leq\left(R_{T}+R_{p}\right)$. This indicates that heavy residues are created in collisions where the center of the projectile lies inside the radius of the target nucleus. This situation is significantly different from the reaction of ${ }^{40} \mathrm{Ar}$ with ${ }^{\mathrm{Nat}} \mathrm{Cu}$ where no target residues were inferred to arise from such central collisions. 9 Therefore, morecentral collisions give rise to heavy residues in these collisions than with lighter nuclei.

Table III-F-1. Total cross section comparison Experimental Large Fragment Cross Section (barns) Calculated Cross Section (barns) Hard Sphere Soft Sphere

$\begin{array}{llll}20 \mathrm{Ne}+181 \mathrm{Ta} & 2.8 \pm 0.5 & 3.64 & 3.47 \\ 20 \mathrm{Ne}+197 \mathrm{Au} & 2.8 \pm 0.5 & 3.80 & 3.59\end{array}$

(D. J. Morrissey, $* *$ W. Loveland, M. de Saint Simon, *** and G. T. Seaborg**) 
Figure III-F-I Product mass distribution for the reaction of $8 \mathrm{GeV}{ }^{20} \mathrm{Ne}+\mathrm{Ta}$ along with similar results from proton induced reactions 3,4 . 


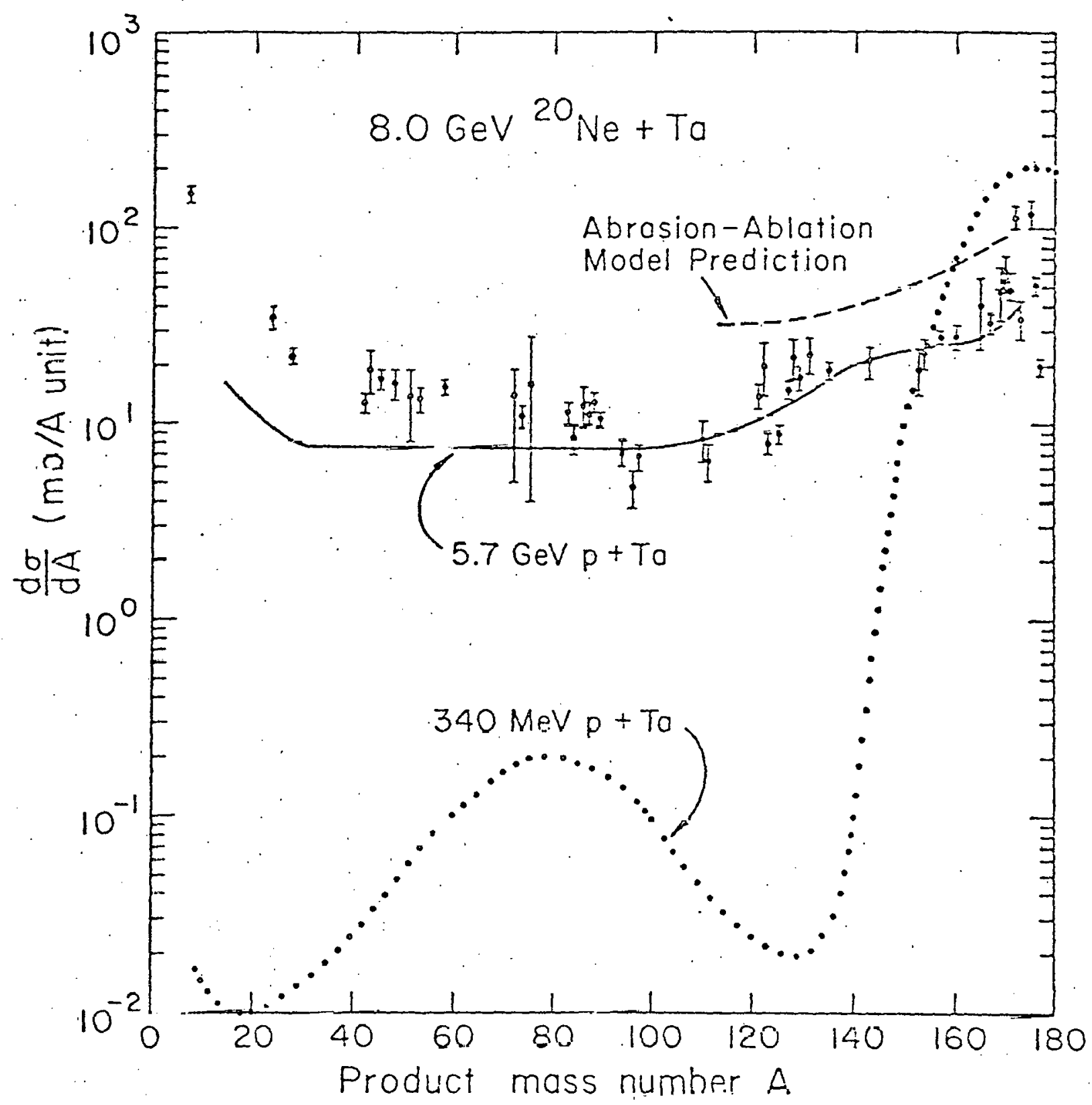

XUL $785-1171$ 
Figure III-F-2 Product mass distributions for the reaction of $8 \mathrm{GeV}{ }^{20} \mathrm{Ne}+\mathrm{Au}$. Also shown are similar results for proton induced reactions 5,6 . 


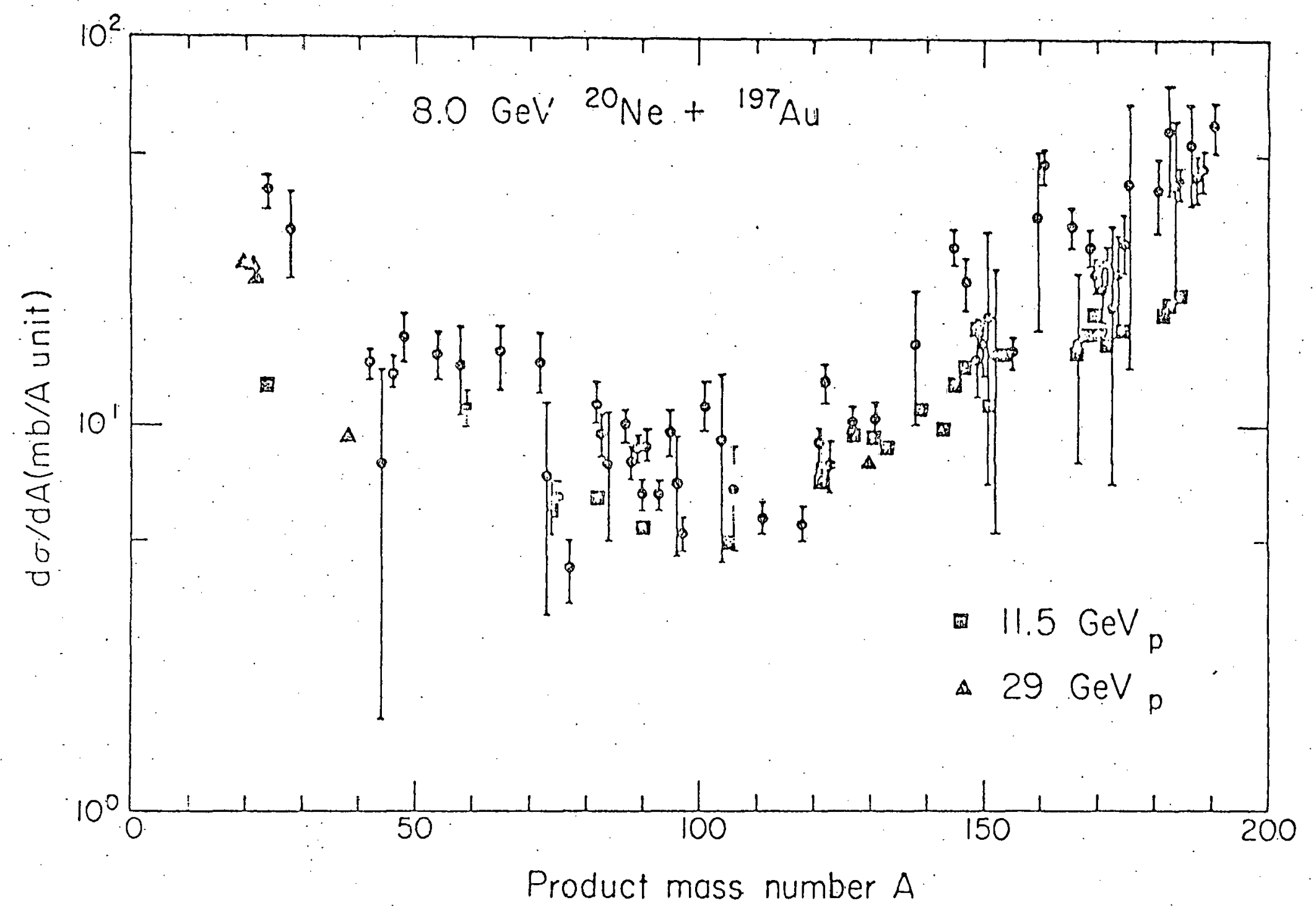

a

$\times 84794-1081$ 


\section{REFERENCES}

* Abstracted from LBL-8983.

** Permanent address, Lawrence Berkeley Laboratory, Berkeley, CA 94720.

*** Permanent address, Laboratorie Rene Bcrnas, BP1, 91406 Orsay, France.

1. See, for exmaple, W. Loveland, R. J. Otto, D. J. Morrissey and G. T. Seaborg Phys. Rev. Lett. 39, 320 (1977).

2. D. J. Morrissey, D. Lee, R. J. Otto and G. T. Seaborg, Nuci. Inst. Meth., 158, 499 (1978).

3. W. E. Nervik and G. T. Seaborg, Phys. Rev. 97, 1092 (1955).

4. J. R. Grover, Phys. Rev. 126, 1540 (1957).

5. S. B. Kaufman, M.H. Weisfield, E. P. Steinberg, B. D. WiTkins and D. Henderson, Phys. Rev. C14, 1121, (1976).

6. J. Hudis, T. Kirsten, R. W. Stoenner, and 0. A. Schaeffer.

7. H. H. Heckman, D. E. Greiner, P. J. Lindstrom and H. Shwe, Phys. Rev. Cl7, 1735 (1978).

8. P. J. Karol, Phys. Rev. C11, 1203 (1975).

9. J. B. Cumming, P. E. Haustein, T. J. Ruth and G. J. Virtes, Phys. Rev. Cl7, 1632 (1978).

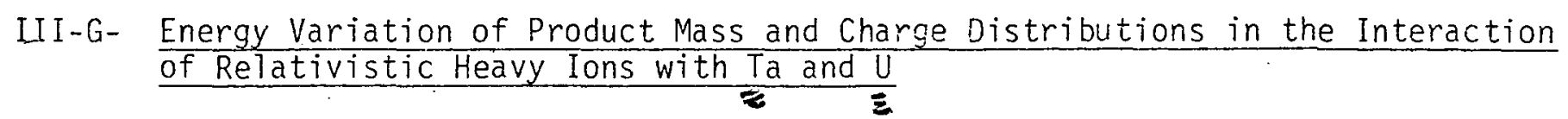

The concept of limiting fragmentation (and the concept of factorization) have been widely applied to explain the product mass charge and momentum distributions for the interaction of relativistic heavy ions (RHI's) with low and intermediate mass targets $\left(\mathrm{Cu}, \mathrm{F}_{\mathrm{g}}\right)^{1,2}$. However, the concept seems to fail when applied to the interaction of RHI's with heavy targets. While the product mass and charge distributions from the interaction of relativistic projectiles with Ta seems relatively insensitive to the projectile energy and identity in the range of 8-25 GeV, (see Figure III-G-1), the product velocity distributions do seem to vary as a function of projectile energy (as can be seen by comparing the results of section III-A of this report and the work of Kaufman, et al. ${ }^{3}$ ). As discussed in Section III-B and III-C of this report, the product mass and charge distributions in the interaction of RHI's with $U$ do not support the use of the concepts of 1 imiting fragmentation and factorization to describe these reactions. 
In hopes of gaining further insight into these questions, we have continued our systematic study of the energy variation of the product mass and charge distributions in the interaction of relativistic heavy ions with typical non-fissionable and fissionable heavy elements, Ta and $U$. We have reported ${ }^{4}, 5,6$ the results of the interaction of $8 \mathrm{GeV}{ }^{20} \mathrm{Ne}$ and $25 \mathrm{GeV}{ }^{12} \mathrm{C}$ with these targets. We have measured the product mass and charge distributions from the interaction of $4.8 \mathrm{GeV}^{12} \mathrm{C}, 5$ and $21 \mathrm{GeV}{ }^{20} \mathrm{Ne}$ with ${ }^{238} \mathrm{U}$ and the interaction of 5.0 and $21 \mathrm{GeV}{ }^{20} \mathrm{Ne}$ and $28 \mathrm{GeV}$ protons with Ta. Analysis of this data is in progress. A final point on these "excitation functions" will be determined in a study of the interaction of $42 \mathrm{GeV} 2 \mathrm{ONe}$ with $\mathrm{Ta}$ and $U$ scheduled for November.

(P. McGaughey, L. L. Nunnelley, D. J. Morrissey, W. Loveland, and G. T. Seaborg)

\section{REFERENCES}

1. J. B. Cumming, R. W. Stoenner, and P. E. Haustein, Phys. Rev. C14, 1554 (1976); see also Phys. Rev. C17, 1632. (1978), Pnys Rev. C10, 739 (1971).

2. C. R. Rudy, and N. T. Ponte, Phys. Lett. 593, 240 (1975).

3. S. B. Kaufillan, E. P. Steinberg, and B. D. Wiikins, Phys. Rev. Lett. 41 , 1359 (1979).

4. D. J. Morrissey, W. Loveland, and G. T. Seaborg, Z. Physik A289, 123 (1978).

5. W. Loveland, R. J. Otto, D. J. Morrissey and G. T. Seaborg, Phys. Rev. Lett.

6. W. Loveland, et a]., Oregon State University Nuclear Chemistry Progress Report RLO-2227-TA35 (1978). 
Figure III-G-1 Product mass distributions for the interaction of relativistic projectiles with Ta. 


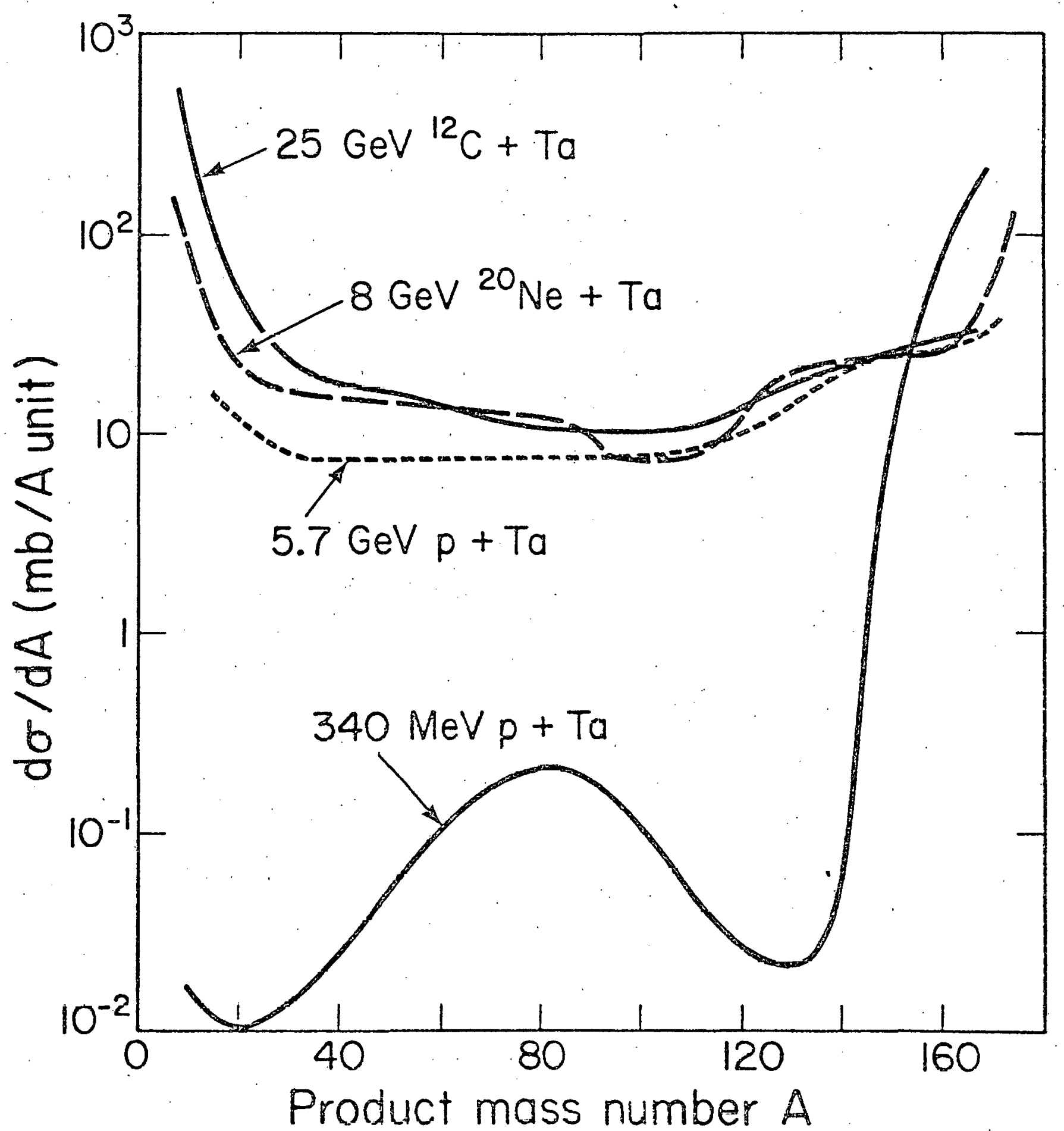

XBL $794-1254$ 
III-H-Product Mass and Charge Distributions in the Interaction of $21 \mathrm{GeV} 20 \mathrm{Ne}$

It has been suggested that relativistic heavy ion (RHI) reactions may serve as useful probes of ground state correlations in nuclear matter. $1-3$ This idea is based upon the premise that RHI reactions occur rapidly relative to the typical time scale of collective nuclear motion. To test this premise and to explore the feasibility of using RHI reactions as probes of nuclear ground staie correlations, we thought it would be of interest to measure the product mass and charge distributions from the interaction of RHI's with rare earth targots.

Ce and Ho targets (of thickness 174 and $114 \mathrm{mg} / \mathrm{cm}^{2}$, respectively) were irradiated with $\sim 10^{13} 21 \mathrm{GeV}{ }^{20} \mathrm{Ne}$ ions at the LBL Bevalac. Gamma-ray spectrometric measurements of the radioactivity induced in the target and catcher foils began approximately one hour after bombardment and continued for five weeks. Approximately 60 radionuclides were identified in each target on the basis of their $\gamma$-ray energy, half-life and radiation abundances. No corrections were made for the contribution to foil activities from secondary induced reactions because previous studies ${ }^{4}$ had shown such corrections to be negligibie for similar targets and experimental conditions.

Independent and cumulative radionuclide yields were calculated from the radioactivity measurements on the irradiated foils. Using procedures described elsewhere, ${ }^{4}$ independent yield formation cross sections were calculated for all radionuclides, Gaussian charge dispersions lof the form $P(Z, A)=\left(2 \pi \sigma^{2}\right)^{-\frac{1}{2}}$ $\left.\exp \left(-(Z-Z p)^{2} / 2 \sigma^{2}\right)\right]$ were fitted to the data, and the charge dispersions were integrated to give the isobaric yields. Figures III-H-I and III-H-2 show the product mass distribution for the reaction of $21 \mathrm{GeV}{ }^{20} \mathrm{Ne}$ with $\mathrm{Ce}$ and $\mathrm{Ho}$, respectiveiy. The general shape of the mass distribution is similar to product distributions measured in the interaction of RHI's with $\mathrm{Ag},{ }^{5} \mathrm{Ta},{ }^{4}$ and $\mathrm{Au} .4$

Figure III-H-3 shows the measured independent yield cross sections for various Tb isotopes from the interaction of $21 \mathrm{GeV}{ }^{20}$ We with ${ }^{15}{ }^{5} \mathrm{Ho}$ along with calculations of those same isotopic yields made using the abrasion-ablation model assuming the charge dispersion is due to zero point oscillations of the giant dipole resonance (GDR). As discussed previously, 4 the abrasion-ablation model overestimates the magnitude of the cross sections and underestimates the excitation energy of these 
near-target residues by some $60 \mathrm{MeV}$, although it does predict approximately the correct width for the charge dispersion.

One interesting question posed previously ${ }^{4}$ is whether statistical de-excitation effects (such as the shape of the mass surface) or the primary product yields (before statistical de-excitation) have the dominant effect on the observed product isotopic distributions. Figure III-H-ه shows the isotonic distribution of products approximately 20 amu removed from a Ce target $(A=120)$ and a Ho target $(A=144)$. Clearly these distributions are quite dissimilar in width al though they result (according to the abrasion-ablation model) from similar primary product distributions. However, comparison of the isotopic distribution for $A=120$ produced from the $\mathrm{Ne}+\mathrm{Ce}$ reaction with the $\mathrm{A}=120$ isotopic distribution produced in the $\mathrm{Ne}+\mathrm{Ho}$ reaction shows remarkable similarity. Thus it would appcar, as discussed previously, that charge distributions of products far removed from the target in mass are dominated by statistical de-excitation effects. Near target residue charge dispersions appear to offer the greatest hope of seeing the primary product distributions unencumbered by de-excitation phenomena.

(R. H. Kraus, Jr., and W. Loveland)

\section{REFERENitS}

1. D. J. Morrissey, W. R. Marsh, R. J. Otto, W. Loveland, and G. T. Seaborg, Phys. Rev. C18, 1267 (1978).

2. L. F. 01 iveria, R. Donange10, and J. 0. Rasmussen, Phys. Rev. C19, 826 (1979).

3. J. P. Bondorf, G. Fai, and 0. B. Nielsen, Phys. Rev. Lett. 41, 391 (1978); Nuc1. Phys. A.312, 149 (1978).

4. See Section III-F of this report.

5. N. T. Porile, G. D. Cole, and C. R. Rudy, Phys. Rev. C19, 2288 (1979). 
Figure III-H-1 Product mass distribution for the reaction of $21 \mathrm{GeV} 20 \mathrm{Ne}$ with Ce. 


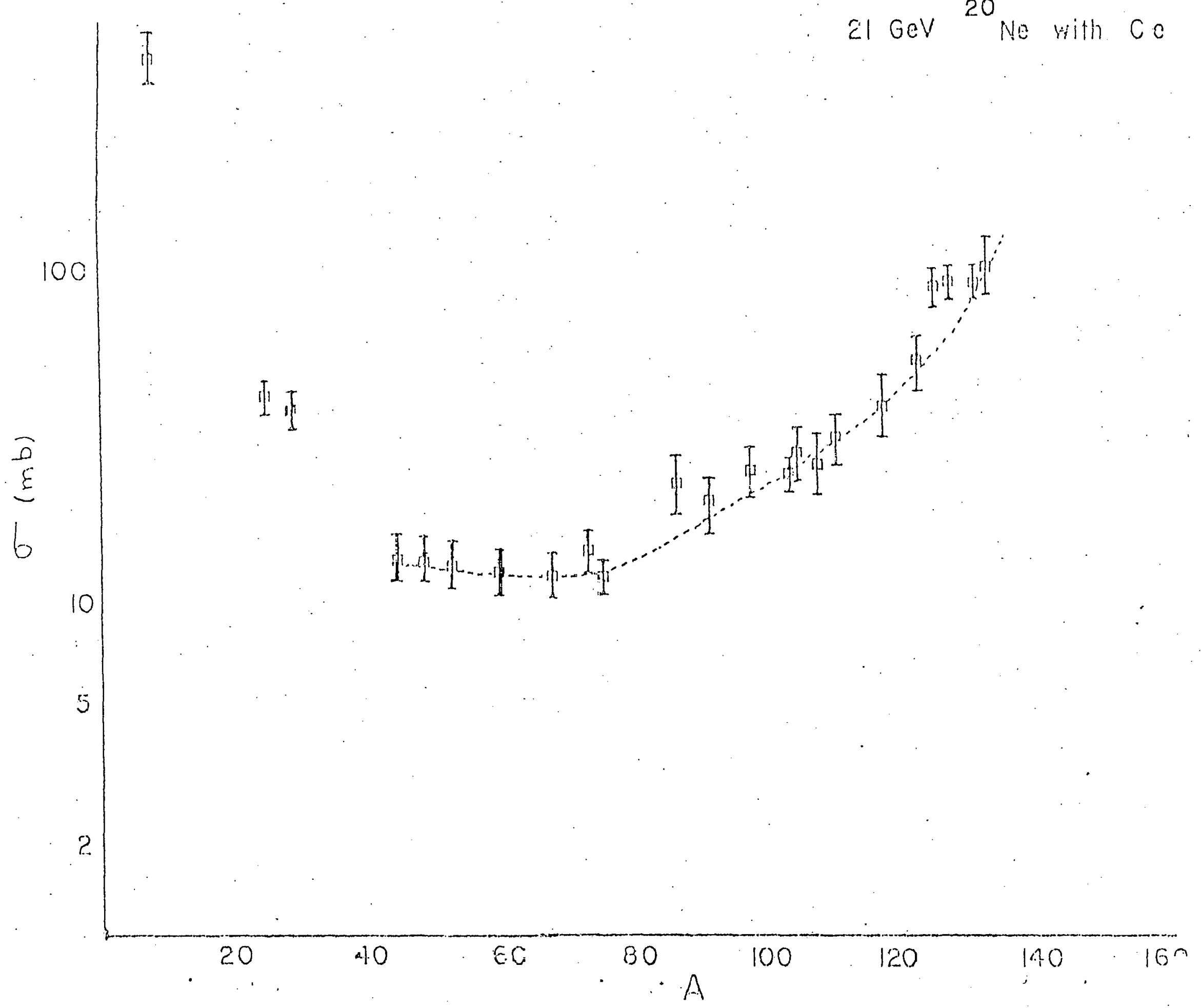


Figure III-H-2 Product mass distribution for the reaction of $21 \mathrm{GeV} 20 \mathrm{Ne}$ with Ho. 


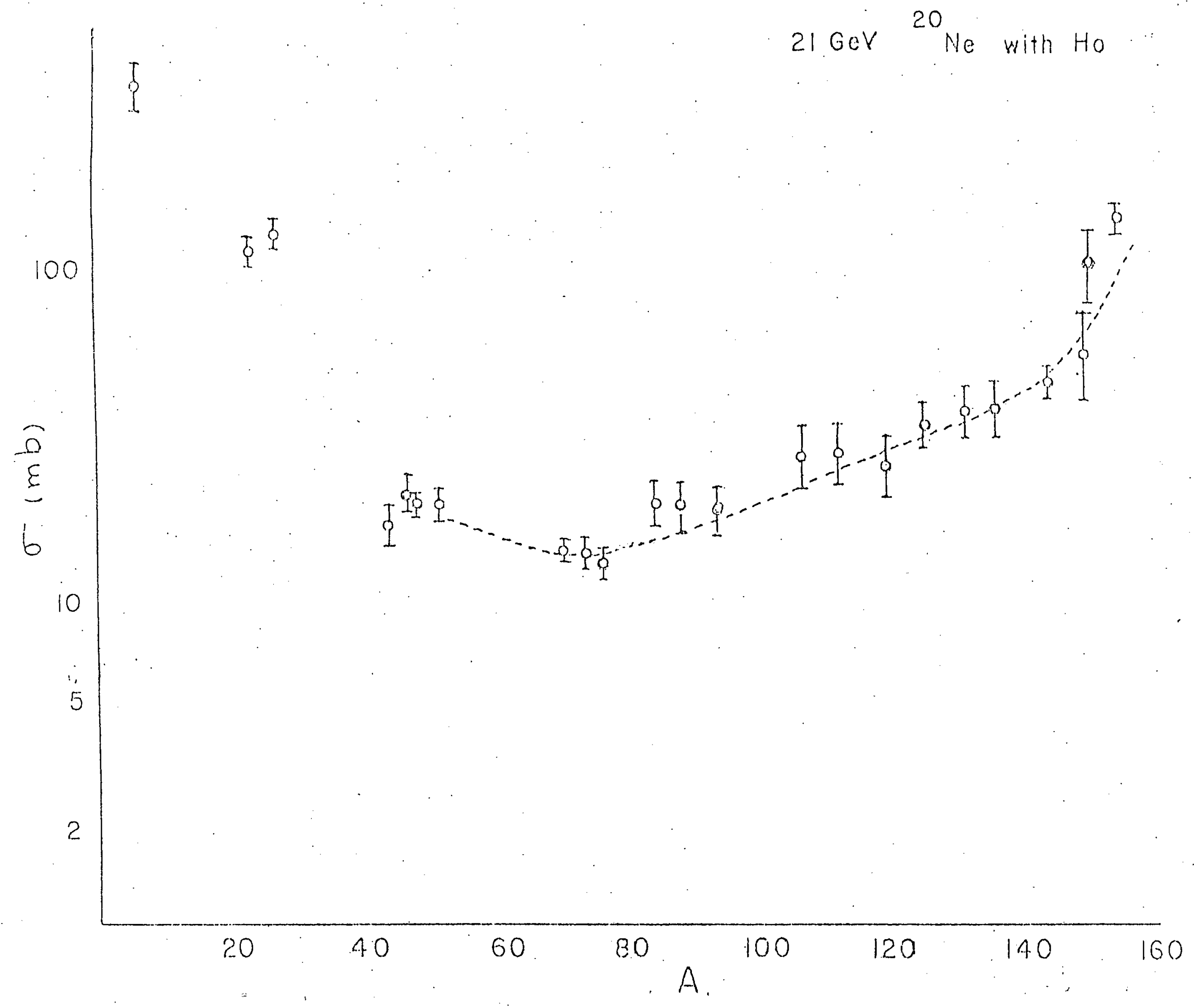


Figure III-H-3 Independent yield cross sections for Tb isotopes produced in the reaction of $21 \mathrm{GeV}{ }^{20} \mathrm{Ne}$ with $\mathrm{Ho}$. 


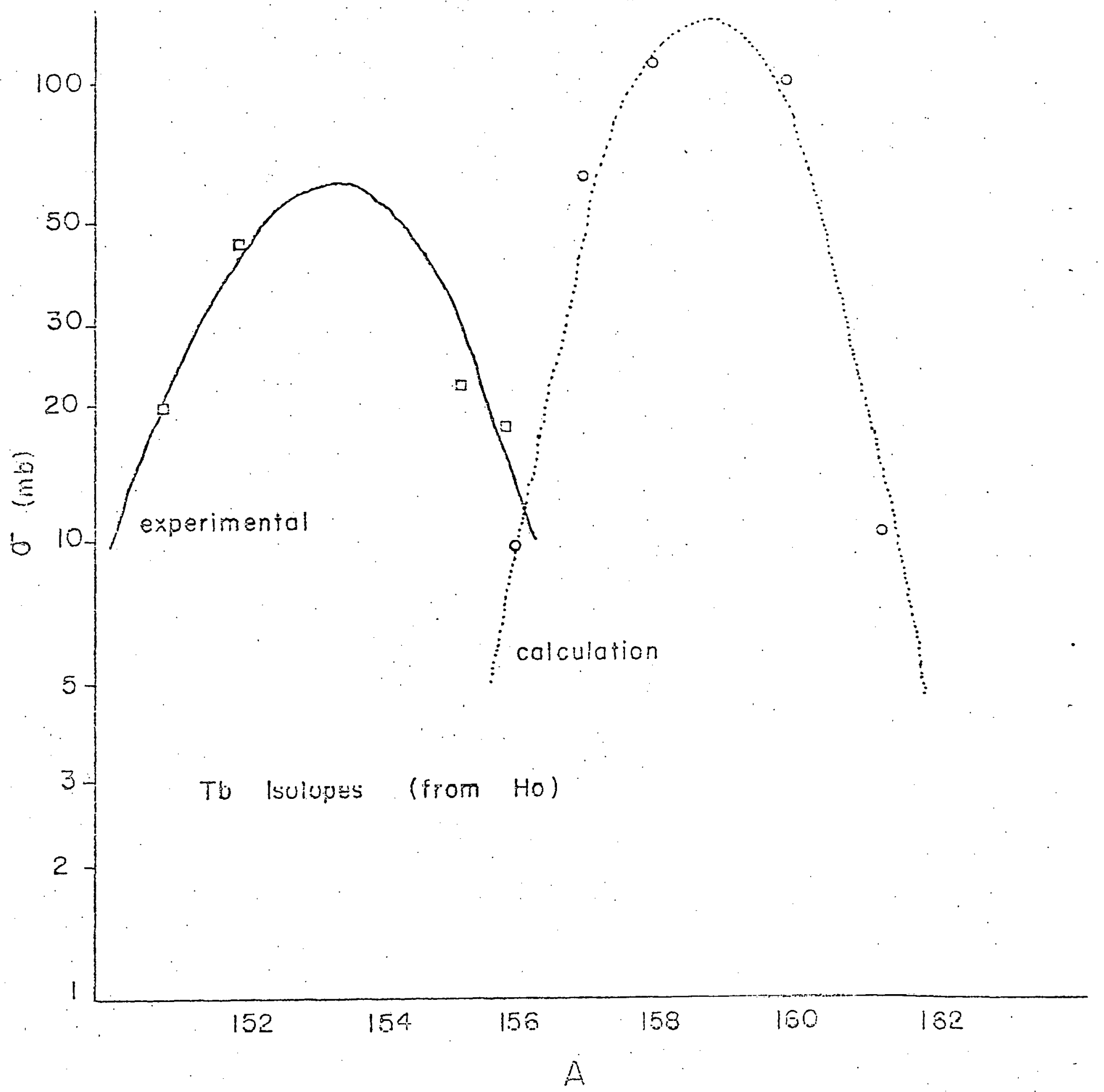


Figure III-H-4 Nuclidic production cross sections for $A=120$ nuclides from the interaction of $21 \mathrm{GeV}{ }^{20} \mathrm{Ne}$ with $\mathrm{Ce}$ and Ho targets are shown along with nuclidic production cross sections for $A=144$ nuclides from the interaction of $21 \mathrm{GeV}^{20} \mathrm{Ne}$ with Ho. 


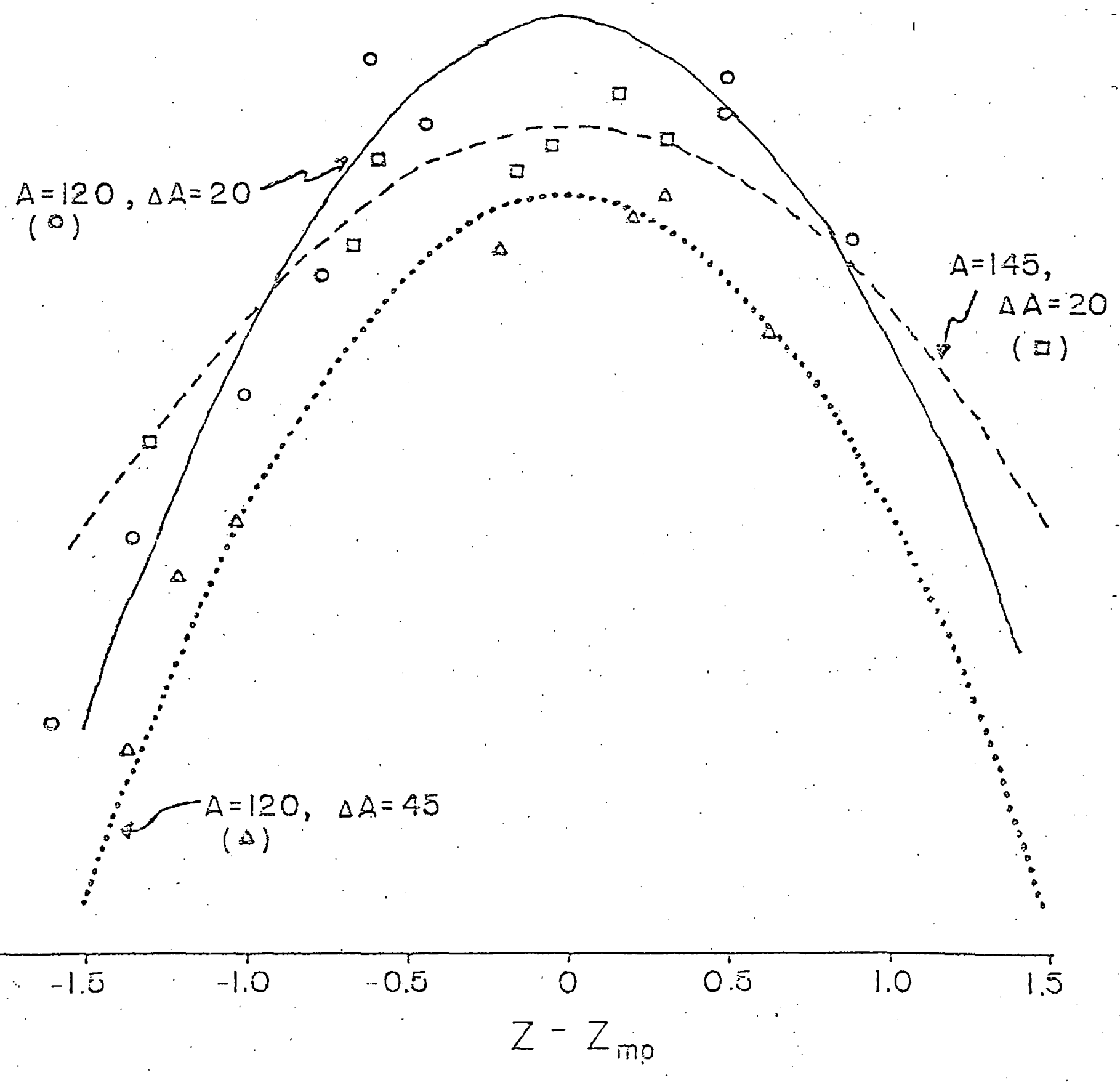


III - I - Relativistic Heavy Ion Induced Fission Fragment Angular Distributions

We wish to report relativistic heavy ion (RHI) induced fission fragment angular distributions for two experiments. On the basis of calculations estimating the angular momentum of the fissioning system and the mass yield curves of similar reaction systems, ${ }^{1,2}$ we outline tentative interpretations of the data.

Thin uranium targets $\left(2600 \mu \mathrm{g} / \mathrm{cm}^{2}\right)$ were irradiated with 8.0 and $21 \mathrm{GeV}$ ${ }^{20} \mathrm{Ne}$ ions at the Lawrence Berkeley Laboratory Bevalac and fragment angular distributions were measured using nuclear track detectors, i.e., CR $-39^{3}$ and Mackofol. Other irradiations $\left(25 \mathrm{GeV}{ }^{12} \mathrm{C}+\mathrm{Pb}, 13 \mathrm{GeV}{ }^{12} \mathrm{C}+\mathrm{U}\right.$ and $5 \mathrm{GeV}$ 'o Ne $+U)$ have been completed with data yet to be analyzed.

The track detector assembly for the $8.0 \mathrm{GeV}{ }^{20} \mathrm{He}$ irradiation allowed us to examine fragments emitted in the forward direction only. The assembly was later improved to enable us to observe fission fragment tracks over the fuil 360 degrees, thereby allowing correlations between forward and backward fission fragment track denisities to be made.

The track detectors were etched several days after irradiation under conditions to maximize development of fission fragment type tracks and minimize low-mass and/or high energy particle tracks. The Mackofol was etched in $6 \mathrm{~N} \mathrm{NaOH}$ at $60-70^{\circ} \mathrm{C}$ and the $\mathrm{CR}-39$ in $10 \mathrm{~N} \mathrm{NaOH}$ at $100-105^{\circ} \mathrm{C}$. These conditions were used to etch track detectors from a ${ }^{2}{ }^{2} \mathrm{Cf}$ chamber calibration exposure yielding high clarity fission fragment tracks with no visible alpha particle tracks.

Figure III-I... shows the fragment angular distributions for the 8.0 and $21 \mathrm{GeV}{ }^{20} \mathrm{Ne}$ experiments. The true intensity of the irradiating beams are unknown, consequentiaily the units of $d \sigma / d \Omega$ are expressed as arbitrary units (au)

A sudden and rapid increase of $d \sigma / d \Omega$ for forward angles is seen in both sets of data. We note that the upswing of $d \sigma / d \Omega$ is of significantly smaller magnitude for the lower energy experiment. There is no evidence of a corresponding increase of do/da for backward angles leading us to conclude that these tracks are not caused by fission fragments. As further support of this idea, we note that the upswing in $\frac{d \sigma}{d \Omega}$ at forward angles is steeper than $\frac{1}{\sin \theta}$. 
One interpretation of the dramatic upswing in this data may be arrived at by considering the radionuclide distributions of two similar experiments: 2,4 $8 \mathrm{GeV}{ }^{20}$ Ne and $25 \mathrm{GeV}{ }^{12} \mathrm{C}$ with uranium targets. The data shows a constant fission cross section for both systems, wile there is a significant increase of heavy collision residues $(150 \leq A \leq 200)$ for the higher energy experiment. This data taken into consideration with the discriminatory etching conditions of our experiment and the forward-peaked nature of the tracks leads us to tentatively conclude the increase of $d \sigma / d \Omega$ at $\theta<30^{\circ}$ is possibly due to heavy residues with kinetic energies on the order of fission fragments.

Finaliy, by fitting the measured fragment angular distributions for the $8 \mathrm{GeV}{ }^{20}$ Ne experiment using a formalism described previously ${ }^{5}$ we deduce a maximum angular momentum of the fissioning system, $I \leq 13 \hbar$.

(R. H. Kraus, Jr., W. Loveland)

\section{REFERENCES}

1. W. Loveland, K. J. Utto, U. J. Morrissey, G. I. Seaborg, Yhys. Lett. 698, $284(19 / 1)$.

2. See Section III-C of this report.

3. Cartright, B. G., E. K. Shirk, P. B. Price, LBL report $\# 7123$.

4. W. Loveland, R. J. Otto, D. J. Morrissey, G. T. Seaborg, Phys. Pev. Lett. 39, 320 (197\%).

5. R. H. Kraus and W. Loveland, Oregon State University Report RLO-2227-TA35 (19\%8). 
Figure III-I-1 Nuclear track angular distributions produced in the interaction of 8 and 21 GeV ${ }^{20} \mathrm{Ne}$ with ${ }^{238} \mathrm{U}$. 


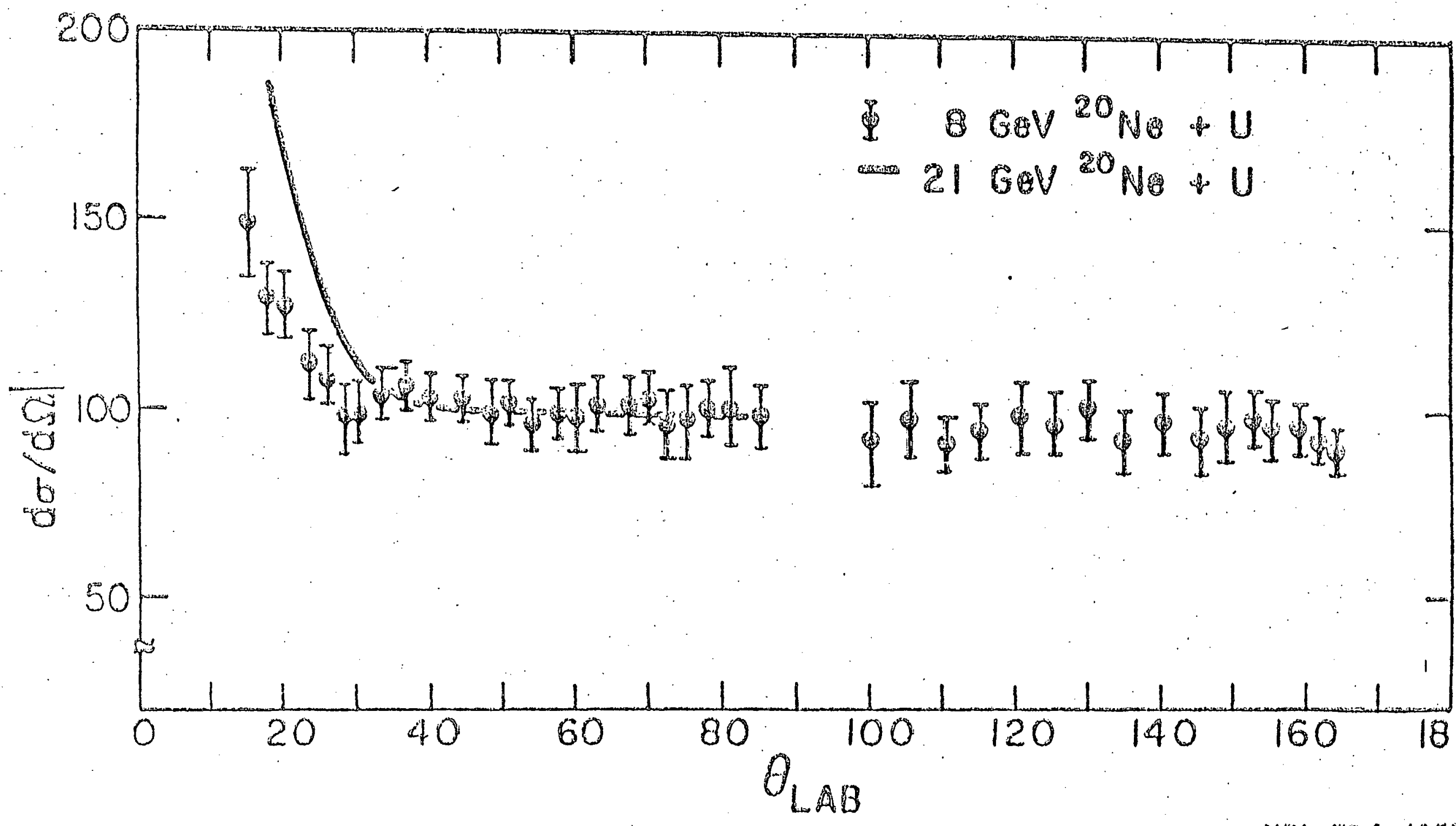

B日L, YQ 
IV. Personnel

W. Loveland - Associate Professor of Chemistry

R. H. Kraus, Jr. - Graduate Research Assistant.

L. L. Nunnelley - Visiting Scientist $(6 / 79-9 / 79)$

Luo Cheng - Visiting Scientist, People's Republic of China (8/79)

V. Publications

A. Articles in Print

1. "New Experimental Insights into the Production of Superheavy Elements Using Heavy Ion Reactions," R. J. Otto, D. J. Horrissey, G. T. Seaborg and .4 . Loveland, in Superheavy Elements, M. A. K. Lodhi, ed. (Pergamon; New York, 1978) pp. 55-72.

2. "Calculation of Target Residue Mass and Charge Distributions in Relativistic Heavy Ion Peactions," D. J. Morrissey, H. R. Marsh, R. J. Otto, W. Loveland, and G. T. Seaborg, Phys. Rev. C18, 1267 (1978).

3. "Implications of Target Residue Mass and Charge Distributions in the Interaction of $8.0 \mathrm{GeV} 20 \mathrm{Ne}+\mathrm{Ta}, " \mathrm{D}$. J. Morrissey, $\|$. Loveland, and G. T. Seaborg, Z. Physik, A289, 123 (1978).

4. "Textbook Errors - Nuclear Beta Decay," W. Loveland, J. Chem. Ed., $56,250(1979)$.

5. "Superheavy Elements - A Crossroads," G. T. Seaborg, H. Loveland, and D. J. Morrissey, Science 203, 711 (1979).

B. Articles in Press

1. "An Interactive Computerized System for the Analysis of Gamma Ray Spectra from Heavy Ion Reactions," D. J. Morrissey, R. J. Otto, D. Lee, J. O. Liljenzin, I. Binder, M. M. Fowler, W. Loveland, and G. T. Seaborg, Proceedings of the International Conference on the Applications of Computers in Activation Analysis and Gamma Ray Spectroscopy.

2. "ReTativistic Heavy Ion Induced Fission of Uranium," H. Loveland, P. McGaughey, D. J. Morrissey, R. J. Otto, R. H. Kraus, Jr., and

G. T. Seaborg, Physics and Chemistry of Fission (IAEA, Vienna).

C. Articles Submitted for Publication

1. "Target Residues from the Reaction of $8 \mathrm{GeV} 2$ Flle with $131 \mathrm{Ta}$ and I97Au," D. 1.) Morrissey. W. Loveland, M. de Saint-Simon, and G. T. Seaborg, Phys. Rev. C (LBL-8983). 
VI. Oral Presentations

1. "Studies of the Target Residues from Relativistic Heavy Ion Reactions with Heavy Element Targets," D. J. Morrissey", G. T. Seaborg, and $H$. Loveland, 176 th ACS National Meeting, lliami Beach, Florida, Sept., 1978.

2. "Superheavy Elements - A Crossroads," H. D. Loveland, D. J. Morrissey and G. T. Seaborg, Bul1. Am. Phys. Soc. 23, 958 (1978).

3. "Relativistic Heavy Ion Reaction Produced Target Residues and the Abrasion Model," D. J. Morrissey, G. T. Seaborg and W. Loveland, Bull. Am. Phys. Soc. 23, 959 (1978).

4. "Radiochemical studies of the Reaction of Relativistic Heavy Ions with Uranium," P. McGaughey, D. J. Morrissey, R. J. Otto, G. T. Seaborg, W. Loveland, and R. H. Kraus. Ir., 177th ACS Natinnal Meeting, Honoiulu, Hawai i, April, 1979.

5. "Product Mass and Charge Distributions from the Reaction of $21 \mathrm{GeV}=:$ :le with Ho and Ce," W. Loveland, R. H. Kraus, Jr., D. J. Morrissey and G. T. Seaborg, 177th ACS National Meeting, Honolulu, Hawai i, April, 1979.

6. "Relativistic Heavy Ion Induced Fission Fragment Angular Distributions," R. H. Kraus, Jr., W. Loveland, D. J. Morrissey, and G. T. Seaborg, 3Ath Northwest Regional Meeting of the ACS, Richland, Uashinoton, June, $19 \overline{79}$.

7. "Entrance Angular Momentum Dependence of the $44 \mathrm{~m}, \mathrm{gSC}$ Isomer Ratio," Aleklett, H. Loveland, D. J. Morrissey, and G. T. Seaborq, 178th ACS National Meeting, Washington, D. C., September, 1979.

\section{Appendices}

At the request of the Pichland Operations Office of the Department of Energy, we have bound copies of reprints and preprints corresponding to work performed during the contract period as part of the Annual Progress Report. 
Relativistic Heavy Ion Induced Fission of Uranium

W. Loveland, P. McGaughey, D. J. Morrissey, R. J. Otto, R. H. Kraus, Jr., and G. T. Seaborg

Lawrence Berkeley Laboratory, Berkeley, CA, U.S.A.

Radiochemical measurements have been made of the product mass and charge distributions from the reaction of $8 \mathrm{GeV}{ }^{20} \mathrm{Ne}$ and $25 \mathrm{GeV}{ }^{12} \mathrm{C}$ with uranium. In addition, we wish to report the fission fragment angular distribution from reaction of $21 \mathrm{GeV}{ }^{20} \mathrm{Ne}$ with uranium. The purpose of these measurements was to help understand the mechanism(s) involved in relativistic heavy ion (RHI) induced nuclear fission and to compare these reactions with fission induced by high energy protons.

The target array for the mass and charge distribution measurements consisted of foils of natural uranium separated from each other by $\sim 150 \mathrm{~mm}$. The foils, varying in thickness from $\sim 25$ to $\sim 75 \mathrm{mg} / \mathrm{cm}^{2}$ and surrounded by $215 \mathrm{mg} / \mathrm{cm}^{2}$ AT catcher foils, were irradiated in RHI beains from the Bevalac for periods from 3 to 18 hours. Gamma-ray spectroscopic measurements of the radioactivity induced in the target and catcher foils began i h after bombardment and continued for about one month. Chemical separations' were used to isolate seven different chemical fractions from one foil and these fractions were assayed by $\gamma$-ray spectroscopy. Over 130 radionuclides were identified in these studies on the basis of their $\gamma$-ray energy, half-life and relative $\gamma$-ray abundance. Based upon the variation of activity with foil thickness, corrections (of $210-30 \%$ ) were made to each measured activity to account for the effects of secondary induced reactions. The corrections vere roughly independent of $A$ with maximum corrections being applied to neutron-rich fission products. Recoil losses from the target were measured to be negligibly small.

Using procedures described previously ${ }^{2}$, independent yield formation cross sections were calculated for all radionuclides, Gaussian charge dispersions [of the form $P(Z, A)=\left(2 \pi \sigma^{2}\right)^{-\frac{1}{2}} \exp \left(-(Z-Z p)^{2} / 2 \sigma^{2}\right)$ ] were fitted to these data, and the charge dispersions integrated to give the yield of each $A$ in the reaction. 
The iodine isotopic distributions from the reaction of $25 \mathrm{GeV}{ }^{12} \mathrm{C}$ with $238 \mathrm{U}$ and $\mathrm{GeV}{ }^{20} \mathrm{Ne}$ with ${ }^{238} \mathrm{U}$ are very similar to each other and to a corresponding iodine distribution in the $2.9 \mathrm{GeV} p$-induced fission of $U$ in that a double-humped charge dispersion is observed with peaks at $\mathbb{H} / \mathrm{Z}$ ul. 5 and $N / Z \sim 1.3$ and width parameters $\sigma=0.8$ and 1.0 , respectively. A detailed examination of the data shows the neutron rich component of the charge dispersion to be nearly identical for the interaction of $p, C$ and $\mathrm{Ne}$ with $U$. The $n$-deficient component of the distribution moves to lower $N / Z$ as one goes from $2.9 \mathrm{GeV}$ protons to $8 \mathrm{GeV}{ }^{20} \mathrm{Ne}$ to $25 \mathrm{GeV}{ }^{2}{ }^{2} \mathrm{C}$. However, the shift in the position of the maximum of this component corresponds to the emission of only two more neutrons, indicating the excitation energy of these species does not change much in going from 2.9 GeV protons to $25 \mathrm{GeV}{ }^{12} \mathrm{C}$ ions as the fission-inducing projectile.

The charge distributions for fission products with $A \sim 100$ are characterized by width parameters $\sigma$ w0.8-1.0 Z units. Direct comparison of these width parameters with those from a number of other high-energy fissioning systems of known excitation energy ${ }^{3}$ allows one to infer the average excitation energy for the fissioning system(s) to be 30-75 HeV.

The fission mass distributions show broad syinmetric peaks extended from $A=70$ to $A=140$, centered around $A=110-115$. From this data we deduce the mean fissioning system mass to be 2225 . Since this mass is greater than the masses of any other reaction products and $\sigma_{f} \sim \frac{1}{2} C R^{4}$, we conclude that fission resulted irom peripheral interactions with impact parameter $b \gtrsim 0.7\left(R_{t}+R_{p}\right)$. Calculations ${ }^{5}$ based upon the abrasion-ablation model of ${ }^{2}$ RHI interactions support this interpretation and are able to quantitatively account for the magnitude of the fission cross section.

In the measurement of the fragment angular distributions, a thin $\left(2660 \mu \mathrm{g} / \mathrm{cm}^{2}\right)$. target of uranium was irradiated with $21 \mathrm{GeV} 2$ ove ions and the fragment angular distribution was measured using nuclear irack detectors (Makrofol and CR-39). The fragment angular distribution for the reaction of $21 \mathrm{GeV}{ }^{2} \mathrm{Ne}$ with $238 \mathrm{U}$ is flat from $\theta_{16}=160^{\circ}$ forward to $\theta_{1}=30^{\circ}$. By comparing the measured fragment angofidr distribution with theoretical expressions ${ }^{5}$, we deduce the mean angular momentum of the fissioning system to be Iav $\leq 13 \hbar$.

\section{REFERENCES}

[1] KRATZ, J.V., LILJENZIN, J.0., SEABORG, G.T., Inorg. Nucl. Chem. lett. 10 (1974) 95.

[2] KRATZ, J.V., LILJENZIN, J.0., NORRIS, A.E., SEABORG, G.T., Phys. Rev. C13 (1970) 2347. LOVELAND, W., MORRISSEY, D.J., UtiU, R.J.,

SEABORG, G.T., Lawrence Berkeley Laboratory Report no. LBL-6527, 1977 (unpublished).

[3] See, for example, FOLGER, R.I., STEVENSON, P.C., SENBORC, G.T., finys. Rev. 98 (1955) 107; MCHUGH, J.A., MICHEL, M.C., Phys. Rev. 172 (1968). 1150 .

[4] KATCOFF, S., HUDIS, J., Phys. Rev. C14 (1976) 628.

[5] MORRISSEY, D.J., MARSH, W.R., OTTO, R.J., LOVELAID, W., SEABORG, G.T., Phys. Rev. C18 (1973) 1267.

[ó] HUIZENGA, J.R., EEHKAili, A.N., MORETTO, L.G., Phys. Rev. 174 (1959)4. 
AN INTERACTIVE COMPUTERIZED SYSTEM FOR THE ANALYSIS OF GAMMA RAY SPECTRA FROM HEAVY ION NUCLEAR REACTIONS*

D. J. Morrissey, R. J. Otto, D, Lee, J, O. Liljenzin, ** I. Binder, ${ }^{\dagger}$ M. M. Fowler, ${ }^{\dagger}$ W. Loveland, ${ }^{+}$and G. T. Seaborg Lawrence Berkeley Laboratory

University of California

Berkeley, California 94720

ABSTRACT

A system for the yield analysis of radionuclides produced in heavy ion nuclear reactions is presented. The system is based on gamma ray spectroscopic measurements of the induced activities, automatic peak identification and fitting with the SAMPO code, and an interactive decay curve identification process. A computer code has been developed which facilitates this process by binding together an updated gamma ray catalogue, the measured decay curves and the ability to fit varying components to the data. 


\section{INTRODUCTION}

Gamma ray spectrometric methods have been developed to deduce mass yield distributions for heavy ion induced nuclear reactions at incident particle energies ranging from $5.0 \mathrm{MeV} / \mathrm{A}$ to $8.5 \mathrm{MeV} / \mathrm{A}$ and 0.4 to $2.1 \mathrm{GeV} / \mathrm{A}$. These nuclear reactions produce radioactive, gamma ray emitting nuclides that cover the entire chart of the nuclides. In a single heavy ion reaction such as $2960 \mathrm{MeV}{ }^{136} \mathrm{Xe}+{ }^{238} \mathrm{U}^{7}$ or $25.2 \mathrm{GeV}{ }^{12} \mathrm{C}+{ }^{298} \mathrm{U}^{2}$ over 100 neutron excessive and neutron deficient nuclides ranging from 'Be to ${ }^{238} \mathrm{~Np}$ were produced and identified by their characteristic gamma ray transitions between $40 \mathrm{keV}$ and $2 \mathrm{MeV}$ from spectrometric measurements of the target.

The object of the analysis is to translate the complicated gamma ray spectra into a data set consisting of the partial cumulative and independent yield production cross sections from which isobaric mass yield distributinns can be dcduced. In the development of a computer aided interactive analysis system the following criteria were established.

a. The energy resolution and the linear and differentlal stability of the speotrometer system should be extremely good. In practice this means we require a system in which the photopeaks, are nearly faussiail in shape and unchanging with time, because single samples have been counted up to one year after the end of bombardment. The absolute efficiency of the detector system has to be well known for the many counting geometries that are used during the spectrometric measurements of different chemical fractions from a single target. 
b. Given the above energy stability and photopeak shape requirements the counting geometry and counting schedule should be adjusted so that production cross section information can be obtained on the greatest number of nuclides, and therefore, the gamma spectrometric measurements should span the largest range of half-lives possible.

c. All routine data handling and processing should be made completely automatic. This includes data acquisition, photopeak analysis and preparation for an interactive decay half-life analysis.

d. In those areas of analysis where a large number of factors must be considered, such as in the assignment of known gamma ray transitions to the measured decay curves of the observed gamma rays, the system, should be an interactive one. It is at this point, also, that the experimenter should be able to evaluate the quality of the data and be able to recognize any systematic errors that the previous automatic part of the analysis may have introduced. 
METHODS

The first criterion above can be met with many commercially available systems today and this aspect will not be discussed. With respect to the second criterion a counting strategy has been developed that takes into account first, the problem of high count rate distortions of the peak shape associated with a very radinartive targct that is cuntinuously decaying, and second, the wide range of half-lives of the gamma ray emitting products in the target. This strategy is based on the obscrvation that the gamma rays from a given radionuclide will only be observable in that period of time when that given radionuclide is going through its second, third and fourth half-lives. That is, a given radionuclide is usually not visible during its first half-life because it will be obscured by short lived activities. It is also not generally visible at longer times because of the promi-. nence of longer lived species.

The gamma fdy spectra are automatically recorded onto magnetic tape along with the start and stop times of the measurement. and an alphanumeric Lay. A typlcail gamma ray spectrum is shown in Figure 1. The identifiration and andigis of photopeaks in the spectra is done with a modified vexsion of the program $\operatorname{sAm}^{3}$. The mudifled automatic mode of this program which was written for the CDC machine at the Lawrence Berkeley Laboratory has proven to be very successful. Desirable features that are built into this code include exact, energy dependent, calibration of the detector efficiency, polynomial energy cali- 
brations, and particularly energy dependent peak line shape calibration. The count rates for each identified peak, corrected for efficiency, are output on magnetic tape in blocks which are labeled with the spectrum tag and the time from the end of bombardment to the midpoint of the measurement.

After the SAMPO analysis is complete the next step of the analysis is to sort the observed gamma ray peak areas so that decay curves can be constructed. For each gamma ray the code TAUl was written to perform this sorting. The code starts with the magnetic tape output from SAMPO and searches first on the spectrum identification tag and then on gamma ray energy. Throughout the analysis the chronological order of the original measurement schedule is preserved. Thus, the SAMPO analysis is performed on spectra in chronological order, which TAUl preserves, thereby eliminating the need for any chronologic sorting in TAUl. The code is able to collect the data from up to 40 spectra for each of 10 samples. The code then generates a new magnetic tape as output that contains the gamma ray intensities sorted by energy for each of the samples. The code also provides a printed output of all the accepted gamma rays for each sample along with the results of the least square estimate of the half-life and the identity of the spectrum in which the gamma ray was observed. The next stage of the analysis is to bring the measured decay curves for each gamma ray together with a compilation of the known gamma ray transitions in order to identify the radio- 
nuclides present in the sample. For this task we have written the computer code TAU2 which is an interactive decay curves analysis program that constructs decay curves and also presents relevant data on the 20 nearest known gamma ray transitions to facilitate the identification. The code has been designed to run on the CDC-6000 machine at LBL with a Tektronix 4014 terminal. Input data for this rnde is the sorted gamma ray data from TAUl and a listing of the updated compilation of Binder et al. 4 of the MacMurdo-Bowman gamma ray tables 5 , both on magnetic tape. The code begins with the lowest energy gamma ray observed in the first sample and plots a semi-logarithmic decay curve, time unit of days, on the CRT of the terminal. Simultaneously the code searches the gamma ray table for a known gamma ray transition nearest to the average measured gamma ray energy. Finding the closest known gamma ray, the code presente thc cnergy, isutupe, half-life, relative intensity and parents (if any) for the 20 gamma rays nearest to the measured energy. A typical display is shown in Figure 2. The operator is then able to choose any single known line or combination of known lines to be least squares fitted to the measured decay curve, or arbitrary half-lives may be fit to the data. The possible combinations are presented in Table 1. When an acceptable identification of the decay curve has been made by the operator the graphical display is recorded on microfiche and the $A_{0}$ value along with its error, energy and radionuclide identification is output on a punched card. This $A_{0}$ value has the units of decays per minute having been corrected for the 
branching ratio of the parent nuclide. Nuclear reaction cross sections are calculated on weighted average of all the observed. gamma rays for each product nuclide after the identifications have been screened for duplicate or erroneous identifications and for self consistency. Once a set of partial cumulative and independent yield cross sections has been obtained an iterative procedure is used to deduce the mass and charge distribution for the nuclear reaction under study. 
RESULTS AND DISCUSSION

We believe that the analysis system that we have described represents a relatively unique combination of analytical methods and programs for the analysis of complex gamma ray spectra. Since no prior knowledge of which radionuclides are contributing to the spectra is required it is hoped that this approach may be applicable to a broad range of problems in the field of activation analysis and gamma ray spectroscopy. Most important to the analysis system that we have developed is the interactive graphics display program which makes it possible to identify reaction products based on their half-lives and known gamma ray transitions. Although this interactive program is presently run on a CDC 6000 machine with a Tektronix 4014 Terminal it could be easily adapted to a smaller computer such as the PDP 1l. Fortran versions of the computer codes described in this work are available. on requect to the authors. 
REFERENCES

*Work supported in part by the Division of Physical Research of the Department of Energy.

**Present address: Department of Nuclear Chemistry, Chalmers University of Technology, Goteberg S, Sweden.

tPresent address: Los Alamos Scientific Laboratory, Los Alamos, New Mexico 87544 .

${ }^{+}$Present address: Oregon State University, Corvallis, Oregon 97331.

1. R. J. Otto, M. M. Fowler, D. Lee and G. T. Seaborg Phys. Rev. Lett. 36,135 (1976).

2. W. Loveland, R. J. Otto, D. J. Morrissey and G. T. Seaborg, Fhys. Rev. Lctt. 39,320 (1977).

3. J. T. Routti and. S. G. Prussin, Nucl. Inst. and Meth. 72, 125 (1969).

4. I. Binder, R. Kraus, R. Klein, D. Lee and M. M. Fowler, Lawrence Berkeley Laboratory Report No. LBL-6515 (1977). 


$$
-10-
$$

5. W. W. Bowman and K. W. MacMurdo, Atomic and Nuclear Data Tables 13, 89-292 (1974). 
TABLE 1

Decay Curve Component Analysis Options.

Single Component

Multiple Components (a) Known gamma ray transition from table

(b) Arbitrary straight line

(a) Known gamma ray plus constant background

(b) Straight line plus constant - background

(c) Sum of two known gamma rays

(d) Sum of known gamma ray plus straight line

(e) Sum of two arbitrary straight lines

(f) growth of known gamma ray from its known parent 
FIGURE CAPTIONS

Fig. 1. Typical gamma ray spectrum observed in this work. A gold target was irradiated with $1140 \mathrm{MeV}{ }^{136} \mathrm{Xe}$ ions and the gamma radiation measured directly. with a range of 75 to $2000 \mathrm{keV}$ in a 4096 channel spectrum.

XBL 776-8998.

Fig. 2. Graphics terminal display showing the fit of two components (solid lines) to the measured decay curve of a $604.4 \mathrm{keV}$ gamma ray (open circles) the ordinate is the logarithm of the count rate and the abscissa the time after the end of bombardment in days. Also shown on the CRT display are 20 gamma rays, nearest in energy to the measured $604.4 \mathrm{keV}$, from the Binder et al. gamma ray catalogue. 4 These entries, numbered 1 to 2.0 , contain. information on the energy, chemical property, isotope, half-life in days, percent abundance of the tranciton and chemical symbol of the parents, if any. XBL $776-8887$. 

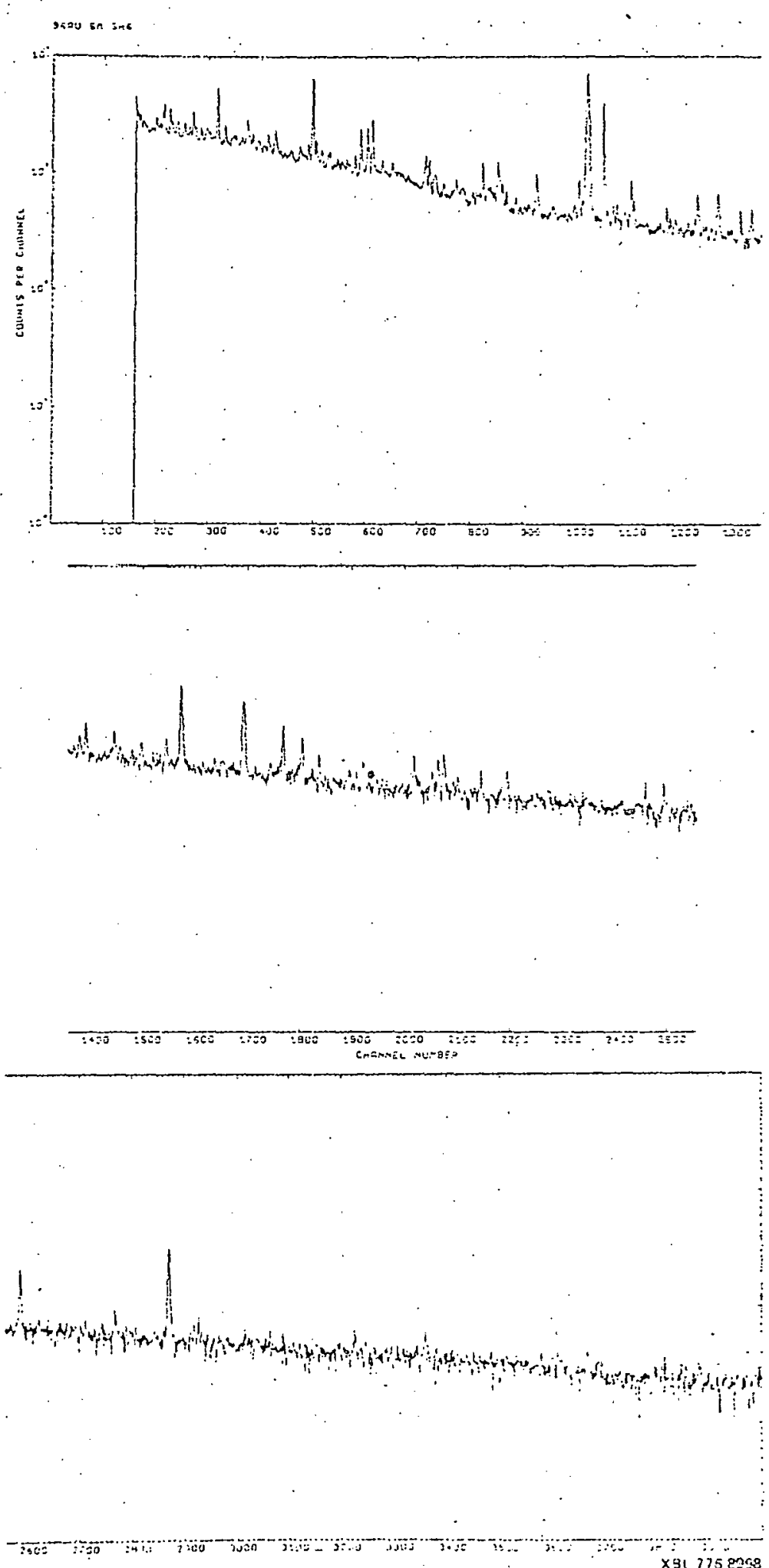


\section{TARGET RESIDUES FROM THE REACTION OF 8 GeV $20 \mathrm{Ne}$} WITH 181 Ta AND ${ }^{197} \mathrm{Au}^{*}$

D. J. Morrissey, W. Loveland $d^{\star \star}$, M. de Saint Simon
and G. T. Seaborg

Lawrence Berkeley Laboratory, University of California Berkeley, CA 94720

\section{ABSTRACT}

The cross sections for the production of 109 different iarget fragments from reactions of $8.0 \mathrm{GeV} 20 \mathrm{Ne}$ with ${ }^{181_{\mathrm{Ta}} \text { and }}{ }^{197} \mathrm{Au}$ were. measured. The target fragment radioactivities were measured by offline gamma ray spectroscopy. Details of the measurement as well as the calculation of the independent isotopic production cross sections $\left(d^{2} c / d Z d A\right)$ and the integrated mass yields $(d \sigma / d A)$ are given. comparisons of these data to previously reported data for proton induced reactions show that the target residue production cross sections scale with the total projectile energy, not velncity. The thital cross section for residue production indicater that some products result from collisions with significant overlap of the central densities of the two nuclei, in contrast to results obtained with low mass targets. Comparisons of the data with a Monte Carlo cascade calculation and an abrasion-ablation calculation verify the importance of ground state correlations of the neutrons and protons on the fragmentation isotopic cross sections. 
NUCleAR REACtions: ${ }^{181} \mathrm{Ta}$ ( ${ }^{20} \mathrm{Ne}$, spallation), ${ }^{197} \mathrm{Au}$ ( ${ }^{20}$ ive, spallation), $E=8.0 \mathrm{GeV} ;$ measured radionuclide production cross sections $\left(\mathrm{d}^{2} \sigma / \mathrm{dZd} A\right)$ and $\mathrm{d \sigma} / \mathrm{dA}$; comparison to proton induced spallation of ${ }^{181_{\mathrm{Ta}} \text { and }}{ }^{197} \mathrm{Au}$; comparison to abrasion-ablation and Monte Carlo cascade models for residue production; $G$ G(Li) spectroscopy, radioanalyticai mass and charge distributions; relativistic heavy ion nuclear reactions. 


\section{INTRODUCTION}

Currently the highest kinetic energy available for the laboratory study of heavy ion induced reactions is $2.1 \mathrm{~A} \mathrm{GeV} .^{1}$ Reactions induced by these heavy ions were thought to hold great promise for the observation of many exotic collective phenomena not observable in proton induced reactions. 2 A large buely of dala now exists from the study of $25 \mathrm{GeV}{ }^{12} \mathrm{C}$ ions interacting with targets ranging from hydrogen to uranium. 3-8 Both rartinehemical and on-line counter techniques have been successfully employed in these studies. The experimental observables have been found to separate into three major categories according to the rapidity of the products: projectile fragments itightiy correlated with the beam rapidity, ${ }^{3}$ from 1 arge impact parameter peripheral. collisions; light products, such as protons, alphas and pi mesons, moving with intermediate rapidities and showing evidence of themalization, 4 from central collisions; and large target residues that do not escape the target materials and thus have low rapidities, ${ }^{5-8}$ from periphera? and near central collisions. These studies have shown no evidence for the predicted exotic phenomena that spurred, thom on, and now the suggestion is that still higher energies are neressary for the obsarvation of such phenomena. 9 Such energies are not presently availabie; however, many facets of the reaction mechanisms at the currently avatiable heavy ion energies are not : yell known. And so, attention is now being turned toward understanding the relationship of heavy ion reactions at a few GeV per nucleon to the well explored fields of low energy heàvy ion reactions and relativistic proton induced reactions. 
The previous studies of relativistic heavy ion (RHI) induced reactions have shown the production, in large amounts, of "heavy" target residues. $5-8,10$ These residues range in mass from essentially that of the target down to the lightest nucleus observable with the radiochemical methods employed, 7 Be. The residue production cross section has been found to be $\sim 70$ percent of the geometrical reaction cross section for both low and high mass targets. Observation of such products is not expected for central collisions of the projectile and target and thus such results set a limit on the number of true central collisions. An important aspect of these studies will be to determine if the number of central collisions, i.e. collisions resulting in the complete annihilation of the target nucleus, is dependent on the projectile velocity. Due to the extreme velocity of the RHI projectiles the total reaction cross section does not vary significantly over this energy region. Up to the present only one study of target residue production in RHI reactions at an energy lower than $2.1 \mathrm{GeV} /$ nucleon has been reported, 11 that for the reaction of $280 \mathrm{MeV} /$ nucleon ${ }^{14} \mathrm{~N}$ with copper. However, only relative yields for target residue products were reported for this system. Thus, the assumption of straight line trajectories that forms the basis of the fireball ${ }^{4}$ and firestreak ${ }^{12}$ models remains. untested over a wide energy region of its assumed applicability. 4,13 .

A second question of basic interest in RHI induced reactions is how such reactions compare with relativistic proton induced reactions. It has become common when discussing the projectile-like and intermediate products from RHI induced reactions to describe the experiments in terms of the projectile velocity (i.e. $2.1 \mathrm{GeV} /$ nucleon, etc.). 3,4,13 
Relative yield measurements by Cumming et al. 5,11 have shown that the target residue isotope production cross sections, $d^{2} \sigma / d Z d A$, for the interaction of $3.9 \mathrm{GeV}{ }^{14} \mathrm{~N}$ and $25.2 \mathrm{GeV}^{12} \mathrm{C}$ with copper targets compare most favorably with isotope production cross sections from reactions induced by protons of equivalent total energy. However, another study by Cumming et al. ${ }^{10}$ showed that the target residue isotope production cross sections from the reaction of $80 \mathrm{GeV}{ }^{40} \mathrm{Ar}$ with copper also compared very favorably with those from the reaction of $28 \mathrm{GeV}$ protons with copper. In view of the observed difference between $25 \mathrm{GeV}{ }^{12} \mathrm{C}$ induced reactions on high mass $8,238 \mathrm{U}$, and low mass ${ }^{5}, \mathrm{Cu}$, targets and the larger volume to surface ratio in high mass targets, we felt it was important to study the relative importance of velocity and total energy in RHI interactions with heavy nuclei. A particularly good target nucleus for this comparison is ${ }^{181} \mathrm{Ta}$. Previous studies of the reaction of $340 \mathrm{MeV}$ protons with ${ }^{181}$ Ta by Nervik and Seaborg, ${ }^{14}$ and the reaction of $5.7 \mathrm{GeV}$ protons with ${ }^{181} \mathrm{Ta}$ by Grover 15 showed large differences between the product mass distributions, do/dA, for these two reactions (see in Fig. 1). The comparison between the mass distribution from the reaction of $8 \mathrm{GcV}{ }^{20} \mathrm{Ne}$ with ${ }^{181}$ Ta with these distributions should allow differentiation between the velocity and total energy dependence of $\mathrm{RHI}$ induced reactions with a ${ }^{181}$ Ta target.

In this paper we report the study of the reaction of $400 \mathrm{MeV} / \mathrm{A}$ ${ }^{20} \mathrm{Ne}$ projectiles $\left(8.0 \mathrm{GeV}\right.$ total energy) with ${ }^{181} \mathrm{Ta}$ and ${ }^{197} \mathrm{Au}$ targets. As discussed above the choice of this projectile and energy allows us to make the comparison with the earlier studies of the proton induced 
reactions and also allows us to study the interaction of RHI at an energy lower than the previously reported studies. In section II we present the details of the experimental procedure used in this work and in section III we present the results. The results are compared to abrasion-ablation model calculations ${ }^{16-18}$ from which we are able to draw conclusions on the ability to observe the predicted ground state correlations of nuclear matter. ${ }^{18,19}$ Part of this work was published previously in a preliminary form. 20 


\section{EXPERIMENTAL}

\section{A. Cross Section Measurement}

As in previously reported radioanalytical measurements of the target residue products from RHI reactions, ${ }^{6-8,14}$ these products are observed through ganma-ray spectroscopy of the target materials subsequent to the irradiation. A detailed review of the reproducibility and sources of error in such measurements has been made by Orth et al. ${ }^{21}$ and the considerations of Cumming ${ }^{22}$ for proton induced reactions certainly apply here. The ${ }^{197} \mathrm{Au}$ targets consisted of two foils, $12.5 \mathrm{~cm}$ by $10.2 \mathrm{~cm}$ each, with thicknesses $49.3 \mathrm{mg} / \mathrm{cm}^{2}$ and $242.0 \mathrm{mg} / \mathrm{cm}^{2}$, each surrounded by $6.6 \mathrm{mg} / \mathrm{cm}^{2}$ Al catcher foils. These two targets, differing by a factor of approximately 5 in thickness, allowed us to evaluate the contribution from secondary particle induced reactions to the RHI reaction products. The ${ }^{181} \mathrm{Ta}$ target consisted of a single foil, $13.6 \mathrm{~cm}$ by $10.2 \mathrm{~cm}$, of thickness $154.1 \mathrm{mg} / \mathrm{cm}^{2}$, also surrounded by $6.6 \mathrm{mg} / \mathrm{cm}^{2}$ Al foils. An external beam of ${ }^{20} \mathrm{Ne}$ was delivered to a $\sim 1 \mathrm{~m}$ air gap by the Bevalac at the Lawrence Berkeley Laboratory. Two separate irradiations were performed, one of the gold foils and one of the tantalum fuil. The average beam fluxes and durations of these irradiations are yiven in Table 1. The bean intensity was monitored on a pulse by pulse basis. with a $\mathrm{Ar}-\mathrm{CO}_{2}$ ion chamber $\left(80 \% \mathrm{Ar}, 20 \% \mathrm{CO}_{2}\right)$ that was developed and calibrated for use at the Bevalac facility. ${ }^{23}$ In order to check the reproducibility of the ion chamber calibration and to facilitate absolute normalization of the calibration, aluminum monitor foils were irradiated in series with the primary target foils and the ionization chamber. 
These aluminum monitor foils were $20.3 \mathrm{mg} / \mathrm{cm}^{2}$ thick and were surrounded by $5.6 \mathrm{mg} / \mathrm{cm}^{2}$ Mylar catcher foils. A schematic diagram of the arrangement of the target foils, monitor foils and ion chamber in the air gap irradiation facility is shown in Fig. 2. The beam enters from the left and passes through the ion chamber, the primary target materials, and then through the Al monitor foil. Approximately $30 \mathrm{~m}$ ahead of the targets the beam is bent $\sigma^{\circ}$ clockwise and approximately $2 \mathrm{~m}$ ahead of the targets it is bent $\sim 10^{\circ}$ further clockwise to remove contaminants from the beam.

Approximate values for the energy loss of the $8.0 \mathrm{GeV}{ }^{20}$ Ne beam in each of the primary targets are given in Table I. The ${ }^{20} \mathrm{Ne}{ }^{10+}$ ions are near minimum ionizing at this energy ${ }^{24}$ and approximate values of the energy loss can be obtained from the Bethe formula. 25,24 As one can see from Table I the energy lost by the ${ }^{20} \mathrm{Ne}$ beam in all the targets is quite small and for the purposes of this work can be ignored. Estimates of the attenuation of the beam by nuclear reactions are also given in Table $I$. These values of $\phi^{\prime} / \phi$, the emergent flux divided by the incident flux, were calculated from the total reaction cross sections of Heckman et al. ${ }^{26}$ Again, these estimates show that the beam is only slightly degraded by passage through the target materials, and therefore nuclear attenuation of the beam also can be ignored. Gamma-ray spectrometric measurements were made with a single $\mathrm{Ge}(\mathrm{Li})$ detector on $\sim 5-10 \mathrm{~cm}^{2}$ area pieces cut from the irradiated target foils, which were centered on the beam spot. These measurements began - 20 minutes after the end of bombardment (EOB) for the gold targets, 14 hours after EOB for the tantalum target, and continued essentially 
uninterrupted for $\sim 40$ days. The identification of the product radioactivities and the calculation of their production cross sections has been described in detail elsewhere, ${ }^{27}$ and will only be described briefly here. After the gamma-ray measurements were completed the spectra were analyzed with an automatic version of SAMPO. 28,27 This code performs a peak-find, a peak-fit and then an energy calculation and a full energy peak efficiency correction for each spertrum. Decay curves arc then . automatically constructed with the sorting routine TAll1. These decay curves are then identified interactively by their gamma-ray energy and half-life with the code TAU2. Multiple assignments are made to the decay curves at this point if the assignment based on the combination of halflife and gamma-ray energy is ambiguous. Off-line, all the assignments are screened to remove multiple assignments and insure that the gammarays for each isotope were observed with the proper abundances (i.e. no transition stronger than the observed transition can be missing). The cross section is then calculated from the statistically weighted average of the observed gamma-ray transitions for each isotope.

The cross section calculation includes corrections for a non-uniform beam level during the irradiation. During the bombardment of the ${ }^{197} \mathrm{Au}$ targets 2.4 percent of the beam was delivered in the first 175 minutes, and the second 97.6 percent was delivered in the.. final 416 minutes, with a 21 minute interruption in between. A constant beam level was maintained during the bombardment of the ${ }^{181}$ Ta target. The production cross sections for all the activities observed in this work are given in Table II. The cross sections and their uncertainties due to counting statistics are given in millibarns for each nuclide 
observed in each of the three targets. The nuclide type, I or $C$, indicates whether the cross section is an independent yield or a partial cumulative yield, respectively. Seventy-seven different radioactivities were observed in the two gold targets (36 in the thinner target and 66 in the thicker target which includes those observed in both) and 74 radioactivities were identified as reaction products in the tantalum target. In all, 109 different radioactivities were identified.

The contribution of secondary particle induced reactions to the measured cross sections can be estimated from a comparison of the production cross sections measured with the $49.3 \mathrm{mg} / \mathrm{cm}^{2}{ }^{197} \mathrm{Au}$ target to those measured with the $242.0 \mathrm{mg} / \mathrm{cm}^{2}{ }^{197}$ Au target. Cumming et al. have previously reported a study of the secondary reaction contributions to the products from the reaction of the $28 \mathrm{GeV}$ protons and $25 \mathrm{GeV}{ }^{12} \mathrm{C}$ ions with copper. ${ }^{5}$ In their study they found that the secondary contributions to cross sections measured using two targets of total thickness $154 \mathrm{mg} / \mathrm{cm}^{2}$ and $1158 \mathrm{mg} / \mathrm{cm}^{2}$ differed by factors that ranged from no contribution to 23 percent with the proton projectiles (the highest contributions were to near-target products). Estimates of the increase in secondary contributions with the ${ }^{12} \mathrm{C}$ projectiles over the protons ranges from a factor of $\sim 1$ to $\sim 3$ when measured with only the thicker copper target. ${ }^{5}$. Novel arguments were used also by Cumming et al . to conclude that secondary. contributions for $80 \mathrm{GeV}{ }^{40} \mathrm{Ar}$ projectiles, for all but the lightest products, were proportional to those found from proton reactions. ${ }^{11}$ The thin target used in the present study was nearly a factor of 3 thinner than the thinnest targets used in obtaining the previously reported estimates of Cumming et al. 5,11 
and secondary contributions in this foil should be negligible.

A simple way to obtain a feeling for the variation and importance of secondary reaction contributions to the cross sections measured with the thicker ${ }^{197} \mathrm{Au}$ foil is to plot the ratio of measurements versus. product mass number. This has been done in Fig. 3 (uncertainties represent one standard deviation), and the values of the ratios are given in Table III for the 24 products observed in both foils. Figure 3 shows that no dramatic variation of the ratio of the measured cross sections can be seen as a function of product mass number. A least squares analysis show's that the linear function $R=1.044-6.0 \times$ $10^{-4} \mathrm{~A}$ gives a slightly better description of the data than a straight line at $R=1$. This function is shown by the dashed line in Fig. 3. The statistical $F$ test of the inclusion of the mass number dependence of the ratio yields a value of $F_{X}=9.2$, which also indicates that such a dependence may be justiried by the data. ${ }^{29}$ However, because all the data points (save two) are within one standard deviation of $R=1$, contributions from secondary reactions to the production cross sections were ignnred.

B. Charye drid Mass Oistributions

The radiochemical cruss sections, whose measurement was described above, represent post neutron and charged particle evaporation, post "fast" beta decay production cross sections. Thus, in order to obtain trup produrtion cross scctions one needs lu currect radiochemically measured cross sections for beta decay, when possible, that occurs between the time of their production in the nuclear reaction and the time at which they are detected through their own beta decay. Once 
corrected these values of the independent production cross sections, $d^{2} \sigma / d Z d A$, can be used to calculate the mass or charge yield, $d \sigma / d A$ or $d \sigma / d Z$ respectively, and also to estimate the total cross section for target residue production.

In order to make the correction for precursor decay to each measured cross section and in order to calculate the total isobaric or mass yield we have used the assumption of Gaussian charge dispersions. That is, the independent yield cross sections can be represented by a histogram that lies along a Gaussian curve, at constant mass number. This is written:

$$
\frac{d^{2} \sigma}{d z d A}=\frac{d \sigma}{d A}\left\{\left(2 \pi s_{z}(A)^{2}\right)^{-1 / 2} \exp \left[\frac{\left(z-z_{p}(A)\right)^{2}}{-2 s_{z}(A)^{2}}\right]\right\}
$$

with the three parameters: $d \sigma / d A$, the total isobaric yield, $s_{z}(A)$, the Gaussian width parameter, and $Z_{p}(A)$ the most probable $Z$ value for that isobar. Given the assumption of Gaussian charge dispersions, the beta decay feeding correction factors for cumulative yield isobaric members can be calculated once the centroid and width of the Gaussian are known.

In order to uniquely specify these three variables $\frac{d \sigma}{d A}, s_{z}(A)$ and $z_{p}(A)$ one would need to measure more than three independent yield cross sections for each isobar. But, there are no isobaric chains that contain three members that are shielded from beta decay. In fact, the nature of radioanalytical studies such as this une dues not, in general, lend itself to the measurement of isobaric members. Rather, a wide assortment of radioactivities are observed which span the entire range of the periodic table that is accessible in the nuclear 
reaction. As a result relatively few isobaric pairs are observed. $5-8,10,11$ A further assumption needs to be introduced in order to apply the Gaussian charge distributions to the measured data. The assumption is, that the value of $d \sigma / d A$ varies smoothly and slowly as a function of mass number, A. This assumption is not as severe as the assumption that the production cross sections for RHT reactions follow the Rudatam systematics 30 for proton induced reactions that has been used in the analysis of data from low mass targets by C.umming et a $7.5,10,11$ Another statement of the former, less stringent, assumption is that the charge dispersion curves for neighboring isobaric. chains should be similar, thus, radionuclide yields from a limited mass range can be used to determine a single charge dispersion curve. The two Gaussian parameters that specify the width, $s_{z}(A)$, and the center $z_{p}(A)$, of the charge distributions are iteratively fit to the measured data over limited mass regions. The width parameter has been fnund, in general, to be approximately independent of mass number over small ranges of $A$. The center of the charge dispersions were adequately represented by linear functions in $A$, over small ranges of $A$, with the exception that an $A^{2}$ term was needed for the highest mass regions. The width parameters and the coefficients of the $Z_{p}(A)$ function are given in Table IV. These parameters should not be considered as absolutes but rather as a consistent set.

The results of this procedure can be seen in Figs. 4 and 5 , where the calculated isotopic production cross sections are plotted versus $Z-Z_{p}(A)$, the distance in $Z$ units from the center of the isobaric charge dispersion. Also shown in Figs. 4 and 5 are the Gaussian curves 
that are specified by equation (1) with the parameters in Table IV, appropriately calculated for histogram distributions in $Z$. The data from the reaction of ${ }^{20} \mathrm{Ne}$ with ${ }^{181} \mathrm{Ta}$ and ${ }^{197} \mathrm{Au}$ are found to lie along the same charge dispersion curves for low mass products, but as one gets closer to the mass of the targets the two distributions separate. An interesting feature of this comparison is that, as shown in Fig. 4 , the low mass products from this reaction have the same charge dispersion width as the same products from RHI reactions with copper targets $10,11,31$ but in this work are shifted towards the neutron excessive side of the valley of beta stability. Such comparisons will be discussed in the next section.

The value of the isobaric or mass yield is obtained for each data point through the assumption of Gaussian charge dispersions and the set of parameters in Table IV. The measured production cross sections are adjusted to remove precursor feeding, where necessary, and a set of independent yield production cross sections, seen in Figs. 4 and 5 , is generated. The mass yield is then calculated as the independent yield cross section divided by the fractional chain yield. The mass yialds obtained in this manner are shown as the solid points in Figs. 6 and 7. 


\section{RESULTS AND DISCUSSION}

It is convenient to divide the presentation of the results into two parts. In the first part the mass distribution results are presented and discussed in terms of their implications for limiting fragmentation, and their relation to the total reaction cross section. In the second part comparisons of the predirtinns of different models of RHI reactions are compared to the detailed isotopic production cross sections for near target residues. These comparisons will shed light on the question of correlations of neutrons and protons in the nuclear ground state.

\section{A. Mass Yield Results}

Comparison of the mass distribution from the reaction of $8.0 \mathrm{GeV}{ }^{20} \mathrm{Ne}$ with ${ }^{181} \mathrm{Ta}$ with the previous studies of proton induced reactions, ${ }^{14,15}$ seen in Fig. 6 , shows the striking agreement of the RHI results with those from proton induced reactions of the (approximately) equivalent total projectile energy. Thts agreement is confirmed by the comparison of the ${ }^{20} \mathrm{Ne}+{ }^{197}$ Au mass distribution with the results of Kaufman et al. ${ }^{32}$ for the reaction of $11.5 \mathrm{GeV}$ protons with ${ }^{197} \mathrm{Au}$ and the sparser results of Hudis et al. ${ }^{33}$ for gaseous products rrum the reaction of $29 \mathrm{GeV}$ protons with ${ }^{197} \mathrm{Au}$. This comparison can be scen in Fig. 7. Although no complete study of the reaction of $\sim 400$ MeV protons with ${ }^{197}$ Au exists, the mass distribution is expected to. be similar to that obtained with ${ }^{181}$ Ta targets. ${ }^{34}$

The agreement of the ${ }^{20} \mathrm{Ne},{ }^{181_{\mathrm{Ta}} \text { arid }}{ }^{197} \mathrm{Au}$ mass distributions with those produced by high energy protons can be viewed as a manifestation of limiting fragmentation, as the studies with copper targets have been viewed. ${ }^{10}$ This process whose origins stem from high energy 
physics, ${ }^{35}$ has been extensively invoked and verified in studies of. projectile fragments in RHI reactions. 26,36 The fact that the general features of the mass distributions from the reaction of $8.0 \mathrm{GeV}{ }^{20} \mathrm{Ne}$ and $29 \mathrm{GeV}$ protons with ${ }^{197} \mathrm{Au}$ are so similar, even though differing widely in projectile energy, would also lend support to the limiting fragmentation hypothesis. However, similarity of these final distribution of products does not necessitate similarity of the primary excitated systems, as suggested in reference 10 from the study of copper fragmentation. On the contrary, Morrissey et al. ${ }^{37}$ have shown that strongly divergent distributions of primary products and excitation energies can, in fact, lead to essentially the same final product distributions for light fragmenting nuclei. This can be attributed to the dominance of the statistical phase of the deexcitation process on the distribution of the final product cross sections in the same way as the momentum distributions of projectile fragments were shown to be dominated by the Fermi momentum. 38 However, these results should be recognized as indicating that little, if any, change can be expected in the mass distribution if the projectile energy is increased.

Integration of the mass yield curves, $d \sigma / d A$, over mass number gives the cross section for the production of target residues. We have chosen to integrate these curves over the interval from mass number 40 to the mass of the target. We have chosen a lower limit of 40 mass units for several reasons, (a) the multiplicity of fragments with masses smaller than 240 is unknown, that is, these products may arise from interactions where another heavy fragment also survives. This certainly is true for fragments such as protons and ${ }^{4} \mathrm{He}$, where 
the number of such fragments per event is much greater than one. ${ }^{4}$ And (b), because in studies such as this one very little data exists for these low mass products.

The results of this integration are contained in Table $V$. The total cross sections for the production of heavy target residues froin ${ }^{181} \mathrm{la}$ and ${ }^{197} \mathrm{Au}$ were found to be $2.8 \pm 0.5$ barns. The fact that a small difference that would be expected on the basis of mass number difference was not seen between the two targets is due to the scatter in the calculated mass yields. For comparison we have included two calculations of the total reaction cross section in Table $V$. The hard sphere calculation refers to the overlap form of the sharp sphere model where the total reaction cross section is written: ${ }^{39}$

$$
\sigma_{R}=\pi r_{0}^{2}\left(A_{T}^{1 / 3}+A_{p}^{1 / 3}-b_{T p}\right)^{2}
$$

We have taken the parameters $r_{0}$ and $b_{T p}$ to be $1.37 \mathrm{fm}$ and $0.51 \mathrm{fm}$, respectively, from the work of Heckman et al. on projectile fragmentation. 26 The soft sphere calculations are those of Karol which were developed to take into account both the diffuse nuclear surface and the variation of the nucleon-nucleon cross section with energy. 40 The comparison of the hard sphere calculntions with uur IIcasured heavy fragment cross sections show that approximately 75 percent of the reaction cross section gives rise to these products. The soft sphere calculation would indicate that these products represent approximately 80 percent of the reaction cross section. 
A current working hypothesis is that target residues are produced in peripheral reactions. ${ }^{17-19}$ An estimate of the impact parameter range that gives rise to these products can be made from the fraction of the total reaction cross section that they represent. Thus, 75 percent of the hard sphere reaction cross section would lie between the impact parameter range of $0.5\left(R_{T}+R_{p}\right) \leqslant b \leqslant\left(R_{T}+R_{p}\right)$. This indicates that heavy residues are created in collisions where the center of the projectile lies inside the radius of the target nucleus. This situation is significantly different from the reaction of ${ }^{40} \mathrm{Ar}$ with ${ }^{\mathrm{Nat}} \mathrm{Cu}$ where no target residues were inferred to arise from such central collisions. 10

B. Comparisons with Reaction Model Calculations

Presently there are two models of the collision process

that occur in RHI reactions that have been used to calculate isotopic and mass yield cross sections. They are a microscopic intranuclear cascade model of Yariv and Fraenke ${ }^{41}$ and the macroscopic abrasionablation model. 16-19 The predictions of these reaction models for the fragmentation of $213 \mathrm{MeV}$ per nucleon $40_{\mathrm{Ar}}$ has shown that the statistical deexcitation of the highly excited primary reaction products plays the dominant role in determining the product mass distributions in both calculations. 37,42 A comparison of these two calculations has also been made for the target residues produced in the reaction of $25 \mathrm{GeV}{ }^{12} \mathrm{C}$ with ${ }^{\mathrm{Nat}} \mathrm{Ag} .{ }^{43,42}$ In this latter comparison, as with the comparison for ${ }^{40} \mathrm{Ar}$, the mass yield curve was well reproduced by both calculations. However, unlike the ${ }^{40}$ Ar fragmentation calculations, the isotopic production cross sections, $d^{2} \sigma / d Z d A$, from the two calculations 
were not at all similar. The Monte Carlo cascade calculation consistently over estimated the width of the isotopic distributions while the abrasionablation calculation ${ }^{42}$ was in reasonable agreement with the data. Therefore, it is interesting to see if the disagreement between the two models increases as the mass number of the fragmenting nucleus is increased.

The collision of the RHI projectile with the target nucleus is trealed as a two step process in the Monte Carlo cascade calculation, a fast step with cascading collisions of nucleons from one reaction partner inside the nucleus of the other partner, and a slow statistical evaporation step of the primary fragments after the fast cascading nucleons have escaped or have been captured by the primary fragments. The calculation is made using an extension of the intranucleon cascade code ${ }^{44}$, VEGAS, for proton induced reactions which has been modified to treat two colliding nuclei. ${ }^{41}$ The calculations were performed with step function density distributions for both nuclei and without refraction and reflection at the nuclear boundaries for the cascading particles. Fermi motion was included in the projectile as well as in the target nucleus. An infinite rearrangement time was assumed for the time neressary for the nuclcus to respond to lhe removal of nucleons from the rermi sea by the fast cascade. Meson production and cascades were included via the ISOBAR model. 45 The impact parameter for each collision was selected at random, and the final production cruss sections were integrated over impact parameter.

The primary fragments from the fast cascade are subsequently individually deexcited using a version of the Dostrovsky, Fraenkel and 
Friedlander statistical model Monte Carlo calculations. 46,41 The excitation energy of each fragment was obtained from the fast cascade code. The absolute value of mass distribution of the final products, i.e. after statistical evaporation, from the reaction of $8 \mathrm{GeV}{ }^{20} \mathrm{Ne}$ with ${ }^{181} \mathrm{Ta}$ is shown in Fig. 8 by the histograms. For clarity the calculated values of the mass yield have been averaged into 5 amu wide bins. The uncertainties shown reflect the uncertainties in the statistics of the calculations and not the uncertainties in the averages.

For comparison with the calculations the measured mass yield results for this system are shown by the dotted curve in Fig. 8 . This curve was drawn smoothly through the data points of Fig. 6 by eye. Several features of the comparison with the cascade calculation are worth noting: (a) the absolute normalization of the cross section for the production of heavy products is approximately correct, but is $225 \%$ too large on the average. (b) The general shape of the residue distribution is also approximately correct in the region above mass number 100. However, below mass number 100 the data and the calculation diverge. The cascade-evaporation calculation first predicts more cross section than is observed (nearly a factor of 2 at $A$ 295) and then drops off to zero cross section before $A \sim 50$.

In the abrasion-ablation view of the collision of the RHI with a target nucleus ${ }^{16-18}$ the two nuclei are taken to be hard spheres which move on straight line trajectories. Those nucleons that lie in the region of overlap of the two nuclei are sheared off in the abrasion (or fast) stage of the collision. The spectator fragments of the target (and projectile) which consist of the nucleons that 
were outside the region of overlap are then assigned an excitation energy that is proportional to their excess surface area. The neutron to proton ratio of the removed nucleons is taken to be equal to that of the target (or projectile) and the variance in the ratio is calculated from the zero point quantum vibrations of the giant dipole resonance (GDR). 18 The oscillator spring constant for this vibration was taken from the liquid droplet model of the GDR. 47 This calculation can be viewed as a leading term approximation to the correlated model of Bondorf et al. 19 in which higher order vibrations are included. The primary products are then allowed to deexcite through a statistical evaporation chain with neutron, proton and alpha emission, and fission competition. 18

The results of thesc calculations are shown in Fig. 8 by the solid line. We have arbitrarily cut off the calculations at $A \backsim 110$ because products with lower mass numbers arise from collisions in which the hard sphere nucleus of the projectile sweeps a cylindrical hole through the target nucleus (removing some 40 nucleons and leaves a "donut-shaped" nucleus with some $\sim 250 \mathrm{MeV}$ of excitation energy due to its increased surface area). It is doubtful whether such nuclei are created and should be considered, rather, as a region outside the limitations of the original model. ${ }^{16}$ As with the cascade calcuitation the absolute magnitude of the mass yield cross section is overestimated by lhe abrasiun-ablation calculation. However, the cross section is over estimated by a factor of $\sim 2$ in the latter case, a much larger discrepancy than that for the former calculation. The general shape 
of the distribution of high mass (i.e. peripheral collision) products is reproduced.

From the comparisons shown in Fig. 8 one can see that both models can predict the general shape of the mass yield curve for high mass products but miss the absolute cross section. Neither calculation can reproduce the mass yields observed for low mass products. That is, neither calculation produces the low mass products observed in the experiment. This discrepancy was hinted at in the study of Porile et al. ${ }^{43}$ with a $\mathrm{Ag}$ target nucleus but is dramatized in the results with Ta target.

A more stringent test of the two reaction models can be made by comparison of the predicted isotopic production cross sections, $d^{2} \sigma / d Z d A$, with the experimental data. A significant difference between the two calculations that is visible in the isotopic cross sections is the amount of correlation of the neutrons and protons that are removed in the fast stage of the reaction. The cascade calculation has no correlations at all as the neutron or proton nature of the cascading nucleons is sclected at random in proportion to their number in the nucleus. On the other hand, the nucleons removed in the abrasion-ablation calculations are highly correlated. 42,18 The difference between the final products of the two calculations. have been shown to be small for low mass nuclei ${ }^{37}$ but should be large in high mass nuclei. Figure 9 shows the calculated final isotropic production cross sections for lutetium and hafnium products from the reaction of $8 \mathrm{GeV}{ }^{20} \mathrm{Ne}$ with ${ }^{181} \mathrm{Ta}$. The irregularities in the curves show the effects of the variation of excitation energy and irregularities in 
the mass surface on the statistical deexcitation process. The difference in the widths of the two distributions is dramatic, especially for these peripheral collision products. This difference stems from both initial the removal of nucleons in an uncorrelated fashion and the delivery to the target nucleus of large amounts of excitation energy with little removed mass by the cascade calculation. Comparison with the experimental data (cf. either Fig. 5 or Fig. 10) shows that the cascade calculation grossly over estimates the widths of the experinluntal isotopic distributions. This must be due, in part, to a lack of correlations in the nucleus. In fact; neither calculation is able to reproduce the measured isotopic distributions although the abrasion-ablation calculation does describe modestly well the width of the distribution. In Fig. $10 \mathrm{~A}$ we present the comparison of the abrasion-ablation calculation to the measured ispotopic distributions for the $\Delta Z=1$ and 2 products from the ${ }^{20} \mathrm{Ne}+{ }^{181}$ Ta system. One sees that the calculations fail to reproduce both the absolute value and the centroid of the distributions. The difference in the absolute normalization was seen before in the comparisons to the mass yield calculations. The difference between the centroids of the calculated and measured distributions may be easily understood.

The excitation energies of the primary fragments produced in the abrasion-ablation model are merely those due to the increased surface area of the fragments. This excitation clearly should be viewed as a lower limit to the true excitation of such fragments. Two very likely sources of excitation of the spectator fragments are any frictional forces acting during the abrasion process and scattering 
of the participant nucleons into the spectator pieces. 17 To explore the magnitude of these excitation energies we have increased the values of the primary fragment excitation energies until the centroids of the calculations match those of the measured products. The results. of this process are shown in Fig. 10A by the solid squares. The primary fragments were raised to a uniform excitation energy of $\sim 75 \mathrm{MeV}$. Extension of these comparisons to products with lower masses was not made because of a lack of data for these products.

In Fig. 108 we show the results of a similar analys is of the near target residues from the reaction of ${ }^{20} \mathrm{Ne}$ and ${ }^{197} \mathrm{Au}$. The features observed with the products from the ${ }^{181}$ Ta target are analogously obtained with the slightly larger target nucleus. In order to match the centroids of the iridium and platinum distributions, the excitation energies of the primary fragments had to be raised to $260 \mathrm{MeV}$. The difference between this value and the value of $\sim 75 \mathrm{MeV}$ for the products from ${ }^{181} \mathrm{Ta}$ may not be significant as it depends on the details of the deexcitation calculation. Thus, we have shown that the near target residues from the reaction of $20 \mathrm{Ne}$ with ${ }^{181_{\mathrm{Ta}}}$ and. ${ }^{197} \mathrm{Au}$ are not accurately described by the Monte Carlo cascade calculation or by the abrasion-ablation calculation. However, the abrasion-ablation calculation can be brought into closer agreement if the excitation energies of the primary fragment are raised to $\sim 60$ to $\sim 75 \mathrm{MeV}$. 


\section{CONCLUSIONS}

This study of the reaction of $8.0 \mathrm{GeV}{ }^{20} \mathrm{Ne}$ with ${ }^{181} \mathrm{Ta}$ and ${ }^{197} \mathrm{Au}$ has shown that several features of the reaction processes occurring in RHI collisions may be best explored with high mass targets. Our results show that the total energy of the projectile is a better parameter for describing the mass yield curve than the projectile. velocity for high mass targets. This is a confirmation of the results of Cumming et a1. obtained with low mass targets. 5,10 This can be viewed as the onset of limiting fragmentation in these reactions, or perhaps more enlightening, as evidence for the dominating effect of the statistical deexcitation process on the mass yield curve. This domination can remove large differences in the distributions of highly excited primary fragments of the initial encounter. ${ }^{37}$ Comparison . of the mass yleld curve to two reaction models, a Monte Carlo intranuclear cascade-mode $7^{41}$ and an abrasion-ablation mode $1^{42}$, shows that netther model predicts the existance of low mass products; although, both generate approximately the correct shape for high mass product distribution. These differences are much more pronounced than those seen in the fragmentation of lighter nuclei. 37,43 Integration of the mass yields for nuclei with $A$ \$40 shows that $\$ 75$ percent of the hard sphere reaction cross section 39,26 ( $\sim 80$ percent of the soft sphere model $\stackrel{40}{*}$ ) is accounted for in such products. This indicates that collisions in which the central density of the projectile lics inside the hard sphere radius of the target contribute to these products. Therefore, more-central collisions give rise to heavy residues in these collisions than with lighter nuclei. Finally, neither reaction model calculation 
was able to reproduce the near-target isotopic production cross sections for either target. In the case of the cascade calculation this may be due to the lack of correlations of the nucleons in the nuclear ground state. The abrasion-ablation calculation fails to reproduce the absolute values of the cross sections as well as the centroids of the isotopic distributions. The former problem may be an indication of the breakdown of the assumption of straight line trajectories in the model, while the latter deficiency has been shown to be due to an underestimation of the excitation energies of the primary fragments. 
ACKNOWLEDGEMENTS

We would like to acknowledge the efforts of Dr. R. J. Otto in the development of the mass yield analysis code and helpful discussions during the course of this work. We would also like to acknowledge the staff of the BEVALAC where the irradiations were performed, especially W. Everette and F. Lothrop.

This work was supported by the U. S. Department of Energy, Nuclear Physics Division, under contract No. W-7405-ENG-48. 


\section{REFERENCES}

* Work supported by the Nuclear Physics Division of the U.S.

Department of Energy.

** Permanent address, Department of Chemistry, Oregon State University, Corvallis, Oregon 97331.

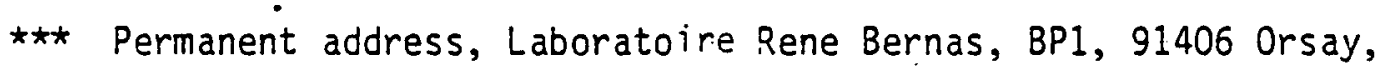
France.

1. H. A. Grunder, W. D. Hartsough and E. J. Lofgren, Science 174, $1128(1971)$.

2. R. Stock and A. M. Poskanzer, Nucl. Part. Phys. 7, 47 (1977).

3. D. E. Greiner, P. J. Lindstrom, H. H. Heckman, Bruce Corfa, and F. S. Bieser, Phys. Rev. Lett. 35, 152 (1975).

4. J. Gosset, H. H. Gutbrod, W. G. Meyer, A. M. Poskanzer, A. Sandoval, R. Stock and G. D. Westfal1, Phys. Rev. C16, 629 (1977).

5. J. B. Cumming, R. W. Stoenner and P. E. Haustein, Phys. Rev. C14, $1554(1976)$.

6. C. R. Rudy and N. T. Porile, Phys. Lett. 598, 240 (1975), and N. T. Porile, G. D. Cole and C. R. Rudy, Phys. Rev. C, subritted for publication, 1979.

7. W. Loveland, R. J. Otto, D. J. Morrissey and G. T. Seaborg, Phys. Lett. $\underline{69 B}, 284$ (1977).

8. W. Loveland, R. J. Otto, D. J. Morrissey and G. T. Seaborg, Phys. Rev. Lett. 39, 320 (1977).

9. N. K. Glendenning and Y. Karant, Phys. Rev. Lett. 40, 375 (1978).

10. J. B. Cumming, P. E. Haustein, T. J. Ruth and G. J. Virtes, Phys. Rev. C17, 1632 (1978). 
11. J. B. Cumming, P. E. Haustein, and R. W. Stoenner, Phys. Rev. C10, 739 (1974).

12. W. D. Myers, Nucl. Phys. A296, 177 (1978).

13. J. Gosset, J. I. Kapusta and G. D. Westfal1, Phys. Rev. C18, 844 (1978).

14. W. E. Nervik and G. T. Seaborg, Phys. Rev. 97, 1092 (1955).

15. J. R. Grover, Phys. Rev. 126, 1540 (1957).

16. J. D. Bowman, W. J. Swiatecki and C. F. Tsang, Lawrence Berkeley Laboratory Report No. LBL-2908, 19/3, unpublished; J. Hufner, K. Schafer and B. Schurman, Phys. Rev. C12, 1888 (1975).

17. J. O. Rasmussen, R. Donagelo and L. F. U1iveira, Proc. IPRC Symp. on Macroscopic Features of Heavy Ion Collisions and Pre-equilibrium Processes, Hakone, 1977 (unpublished); L. F. Oliveira, R. Donangelo, and J. 0. Rasmussen, Phys. Rev. C19, 826 (1979).

18. D. J. Morrissey, W. R. Marsh, R. J. Otto, W. Loveland and G. T. Seaborg, Phys, Rev. C18, 1267 (1978).

19. J. P. Bondorf, G. Fai and O. B. Nielsen, Phys. Rev. Lett. 41, 391 (1978); Nucl. Phys. A312, 149 (1978).

20. D. J. Morrissey, W. Lovel and and G. T. Seaborg, Z. Phys. A289, 123 (1978).

21. C. J. Orth, H. A. O'Brien Jr., M. E. Schillaci, B. J. Dropesky, J. E. Cline, E. B. Nieschmidt, and R. L. Brodzinski, J. Inorg. Nucl. Chem 38, 13 (1976).

22. J. B. Cumming, Ann. Rev. Nucl. Sci. 13, 261 (1963).

23. W. L. Everette, private communication.

24. L. C. Northcliffe and R. F. Schilling, Nucl. Data Tables $\underline{7}$, $233(1970)$. 
25. L. C. Northcliffe, Ann. Rev. Nucl. Sci. 13, 67 (1963).

26. H. H. Heckman, D. E. Greiner, P. J. Lindstrom and H. Shwe, Phys. Rev. C17, 1735 (1978).

27. D. J. Morrissey, D. Lee, R. J. Otto and G. T. Seaborg, Nucl. Inst. Meth., 158, 499 (1978).

28. J. T. Routti and S. G. Prussin, Nucl. Inst. Meth. 72, 125 (1969).

29. See, for example, P. R. Bevington, Data Reduction and Error Analysis for the Physical Sciences (McGraw-Hill, New York, 1969), p. 200.

30. G. Rudstam, Z. Naturforsch. 21a, 1027 (1966).

31. P. E. Haustein and T. J. Ruth, Phys. Rev. C18, 2241 (1978).

32. S. B. Kaufman, M. W. Weisfield, E. P. Steinberg, B. D. Wilkins and D. Henderson, Phys. Rev. C14, 1121, (1976).

33. J. Hudis, T. Kirsten, R. W. Stoenner, and 0. A. Schaeffer, Phys. Rev. C1, 2019 (1970).

34. See, for example, V. E. Ross and K. Bachmann, J. Inorg. Nucl. Chem. 21,13 (1974), and references therein.

35. H. Boggild and T. Ferbel, Ann. Rev. Nucl. Sci. 24, 451 (1974).

36. See, for example, H. H. Heckman, H. J. Crawford, D. E. Greiner, P. J. Lindstrom, and L. W. Wilson, Phys. Rev. C17, 1651 (1978); and G. D. Westfall, L. W. Wilson, P. J. Lindstrom, H. J. Crawford, D. E. Greiner and H. H. Heckman, Phys. Rev. C19, 1309 (1979); and references therein.

37. D. J. Morrissey, L. F. Oliveira, J. O. Rasmussen, G. T. Seaborg, Y. Yariv and Z. Fraenkel, Lawrence Berkeley Laboratory Report No. LBL-8964, 1979, submitted for publication.

38. H. Feshbach and K. Huang, Phys. Lett. 47B, 300 (1973). 
39. H. L. Bradt and B. Peters, Phys. Rev. 77, 54 (1950).

40. P. J. Karol, Phys. Rev. C11, 1203 (1975).

41. Y. Yariv and Z. Fraenkel, to be published.

42. The specific form of the abrasion-ablation model used in the comparison was that of reference 18.

43. N. T. Porile, G. D. Cole and C. R. Rudy, Phys. Rev. C, submitted for publication, 1979.

44. K. Chen, Z. Fraenkel, G. Friedlander, J. R. Grover, J. M. Miller, and Y. Shimamoto, Phys. Rev. 166, 949 (1968).

45. G. D. Harp, K. Chen, G. Friedlander, Z. Fraenkel, and J. M. Miller, Phys. Rev. C8, 851 (1973).

46. I. Dostrovsky, Z. Fraenkel and G. Friedlander, Phys. Rev. $\underline{116}$, 683 (1959).

47. W. U. Myers, W. J. Swiatecki, T. Kndama, L. J. El-Jaick, and E. R. Hilf, Phys. Rev. C15, 2032 (1977). 


\section{FIGURE CAPTIONS}

Fig. 1. Large differences in the product mass yield (do/dA) obtained as a function of incident proton energy are shown by the work of Nervik and Seaborg ${ }^{14}$ (340 MeV) and Grover ${ }^{15}$ (5.7.GeV).

Fig. 2. A schematic diagram of the target arrangement used in the bombardment with ${ }^{20} \mathrm{Ne}$ projectiles at the BEVALAC is shown.

Fig. 3. The ratio of the production cross sections measured with the thick ${ }^{197}$ Au target to those measured with the thin ${ }^{197} \mathrm{Au}$ are plotted versus the radionuclides' mass number. The solid line at $R=1$ represents the value of this ratio that would be expected if there was no contribution from secondary particle induced reactions. The dashed curve is discussed in the text.

Fig. 4. A comparison of the charge dispersion curve for the low mass products, $30 \leqslant A \leqslant 50$, observed in this work (solid curve) with the charge dispersion curve previously measured for products in the same mass range produced in the reaction of $80 \mathrm{BeV}^{40} \mathrm{Ar}, 3.9 \mathrm{GeV}{ }^{14} \mathrm{~N}, 3.9 \mathrm{GeV}{ }^{1} \mathrm{H}$ and $1.57 \mathrm{GeVII}$ - with copper 10,11,31 (dotled curve). The latter curve has been arbitrarily normalized to convert the reported values of fractional chain yields into isotopic production cross sections, $d^{2} \sigma / d Z d A$. 
Fig. 5. The charge dispersion curves obtained in this work are shown as a function of mass region of the products. The parameters that describe the center and width of the individual curves are given in Table IV.

Fig. 6. The target residue mass distribution, do/dA, obtained for the reaction of relativislic prujecliles with a ${ }^{181}$ Ta target is shown.

Fig. 7. Similar to Fig. 6, the mass distribution ohtained for the $8 \mathrm{GeV}{ }^{20} \mathrm{Ne}+{ }^{197} \mathrm{Au}$ target is plotted. For comparision, the data of Kaufman et. al. ${ }^{32}$ for 11.5 GeV protons (solid squares) plus ${ }^{197} \mathrm{Au}$ and the data of Hudis et al. ${ }^{33}$ for $29 \mathrm{GeV}$ protons plus ${ }^{197} \mathrm{Au}$ (solid triangles) are also shown.

Fig. 8. A comparison of the mass yield curve measured in this work (dotted) with the predictions of the Monte Carlo cascade model $^{41}$ (histograin) and with the predictions of the abrasionablation model ${ }^{42}$ (solid curve).

Fig. 9. Calculated final product isotopic distributions are shown for the reaction of $20 \mathrm{Ne}+{ }^{181} \mathrm{Ta}$ to produce lutetium and hafnium 15otopes. The solid line is from the abrasion-ablation model ${ }^{42}$ and the histograms from the cascade model. 11 
$-32-$

Fig. 10. The isotopic production cross sections from the abrasionablation model (triangles) are compared to the measured data (solid points) for the reaction of ${ }^{20} \mathrm{Ne}$ with ${ }^{181} \mathrm{Ta}$, (A), and ${ }^{197} \mathrm{Au},(B)$. The results for the deexcitation of more highly excited primary products as discussed in the text are shown for both target nuclei (solid squares).

Reprints

Removed 
Table I. Irradiation conditions, target thickness and approximate values of the beam degradation by the target foils.

\begin{tabular}{|c|c|c|c|c|c|}
\hline Target & $\begin{array}{c}\text { Foil } \\
\text { Thickness } \\
\left(\mathrm{mg} / \mathrm{cm}^{2}\right) \\
\end{array}$ & $\begin{array}{l}\text { Average Flux } \\
\text { (particles/min) }\end{array}$ & $\begin{array}{l}\text { Length of } \\
\text { Boimbardment } \\
\text { (minutes) }\end{array}$ & $\begin{array}{c}\text { Energy Loss } \\
\text { in Target } \\
\text { MeV (percent) }\end{array}$ & $\begin{array}{c}\text { Nuclear Reaction } \\
\text { Attenuation } \\
\phi \% \phi \\
\end{array}$ \\
\hline \multirow[t]{2}{*}{$A u-I$} & 49.3 & & & $<15(<0.2)$ & 2.9994 \\
\hline & & $7.19 \times 10^{9}$ & 612 & & \\
\hline $\mathrm{Au}-\mathrm{II}$ & 242.0 & & & $<60(<0.7)$ & $\sim .997$ \\
\hline $\mathrm{Ta}$ & 154.1 & $1.64 \times 10^{10}$ & 247. & $<40(<0.5)$ & $\sim .998$ \\
\hline
\end{tabular}


$-34-$

Table II. Cross Sections (in millibarns) observed in this work.

\begin{tabular}{|c|c|c|c|c|}
\hline Nuclide & Type ${ }^{a}$ & $49.3 \mathrm{mg} / \mathrm{cm}^{2} \mathrm{Au}$ & $\frac{\text { Target }}{242 \mathrm{mo} / \mathrm{cm}^{2} \mathrm{Au}}$ & $154 \mathrm{ma}^{\mathrm{cm}^{2} \mathrm{Ta}}$ \\
\hline $7_{\mathrm{Be}}$ & c & -- & -- & \pm 2.5 \\
\hline${ }^{24} \mathrm{Na}$ & C & -- & \pm 3.3 & $28.9 \pm 2.9$ \\
\hline${ }^{28} \mathrm{Mg}$ & c & $7.5 \pm 2.0$ & --- & $5.7 \pm 0.75$ \\
\hline $41_{\mathrm{Ar}}$ & c & $18.3 \pm 1.8$ & $17.8 \pm 1.8$ & --- \\
\hline $42_{k}$ & I & --- & $9.04 \pm 0.9$ & $7.4 \pm 0.7$ \\
\hline $43_{K}$ & c & -- & --- & $54.5 \pm 14.4$ \\
\hline${ }^{44} \mathrm{Sc}$ & I & --- & $1.9 \pm 1.5$ & --- \\
\hline${ }^{44 m} \mathrm{Sc}$ & I & -- & --- & $3.2 \pm 0.3$ \\
\hline${ }^{46} \mathrm{Sc}$ & I & --- & $9.3 \pm 0.9$ & $14.8 \pm 3.2$ \\
\hline${ }^{48} \mathrm{SC}$ & I & $2.8 \pm 0.4$ & $2.8 \pm 0.1$ & $2.4 \pm 0.4$ \\
\hline $48 v$ & C & --- & $3.1 \pm 0.3$ & $3.1 \pm 0.3$ \\
\hline${ }^{51} \mathrm{Cr}$ & C & --- & -- & $6.9 \pm 2.8$ \\
\hline${ }^{52} \mathrm{Mn}$ & C & --- & --- & $1.66 \pm 0.11$ \\
\hline${ }^{54} \mathrm{Mn}$ & I & --- & $9.0 \pm 1.3$ & $10.6 \pm 1.4$ \\
\hline${ }^{58} \mathrm{Co}$ & I & -- & $5.7 \pm 1.4$ & $8.7 \pm 0.9$ \\
\hline${ }^{59} \mathrm{Fe}$ & c & --- & $2.8 \pm 0.2$ & $2.3 \pm 0.2$ \\
\hline${ }^{65} \mathrm{Zn}$ & C & -- & $7.0 \pm 1.4$ & $--\cdot$ \\
\hline${ }^{72} \mathrm{Ga}$ & c & -- & --- & $3.1 \pm 1.8$ \\
\hline${ }^{72}$ As & $c$ & $6.7 \pm 0.6$ & $7.6 \pm 1.2$ & -- \\
\hline${ }^{73} \mathrm{Se}$ & C & $3.6 \pm 2.0$ & -- & $1.8 \pm 0.1$ \\
\hline${ }^{74}$ As & I & $5.2 \pm 0.9$ & -- & $4.8 \pm 0.6$ \\
\hline${ }^{75} \mathrm{Se}$ & C & -- & $6.4 \pm 0.4$ & $7.7 \pm 6.1$ \\
\hline${ }^{76}$ As & I & -- & -- & $9.0 \pm 0.9$ \\
\hline${ }^{77} \mathrm{Br}$ & c & $\cdots$ & $4.3 \pm 0.8$ & $3.5 \pm 0.3$ \\
\hline $82 m_{R b}$ & I & --- & $6.4 \pm 0.8$ & --- \\
\hline $83_{\mathrm{Rb}}$ & C & -- & $8.5 \pm 1.2$ & $8.7 \pm 0.9$ \\
\hline${ }^{84} \mathrm{Rb}$ & I & $3.8 \pm 1.4$ & $4.1 \pm 0.3$ & $2.2 \pm 0.3$ \\
\hline
\end{tabular}


Table II. (continued)

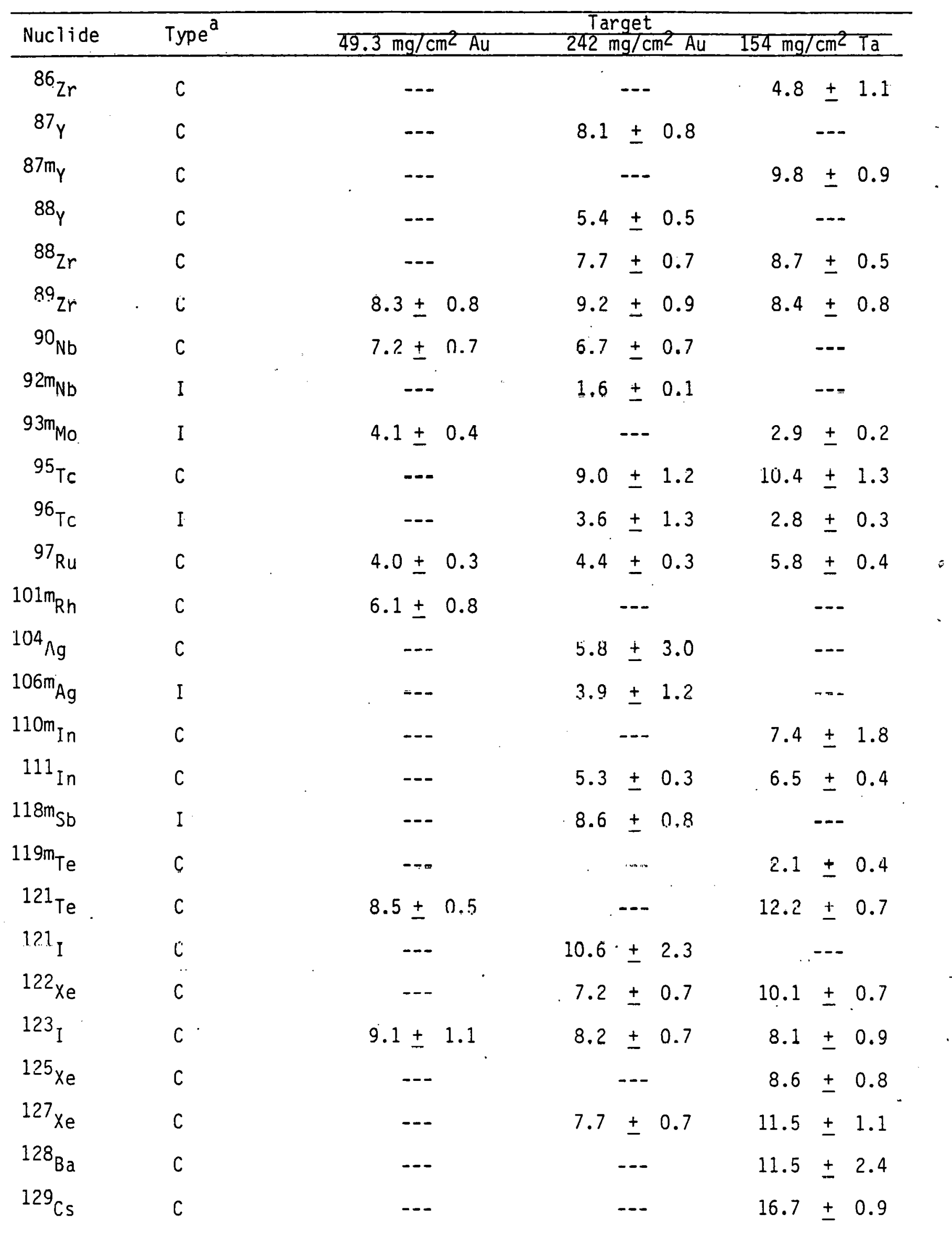


Table II. (continued)

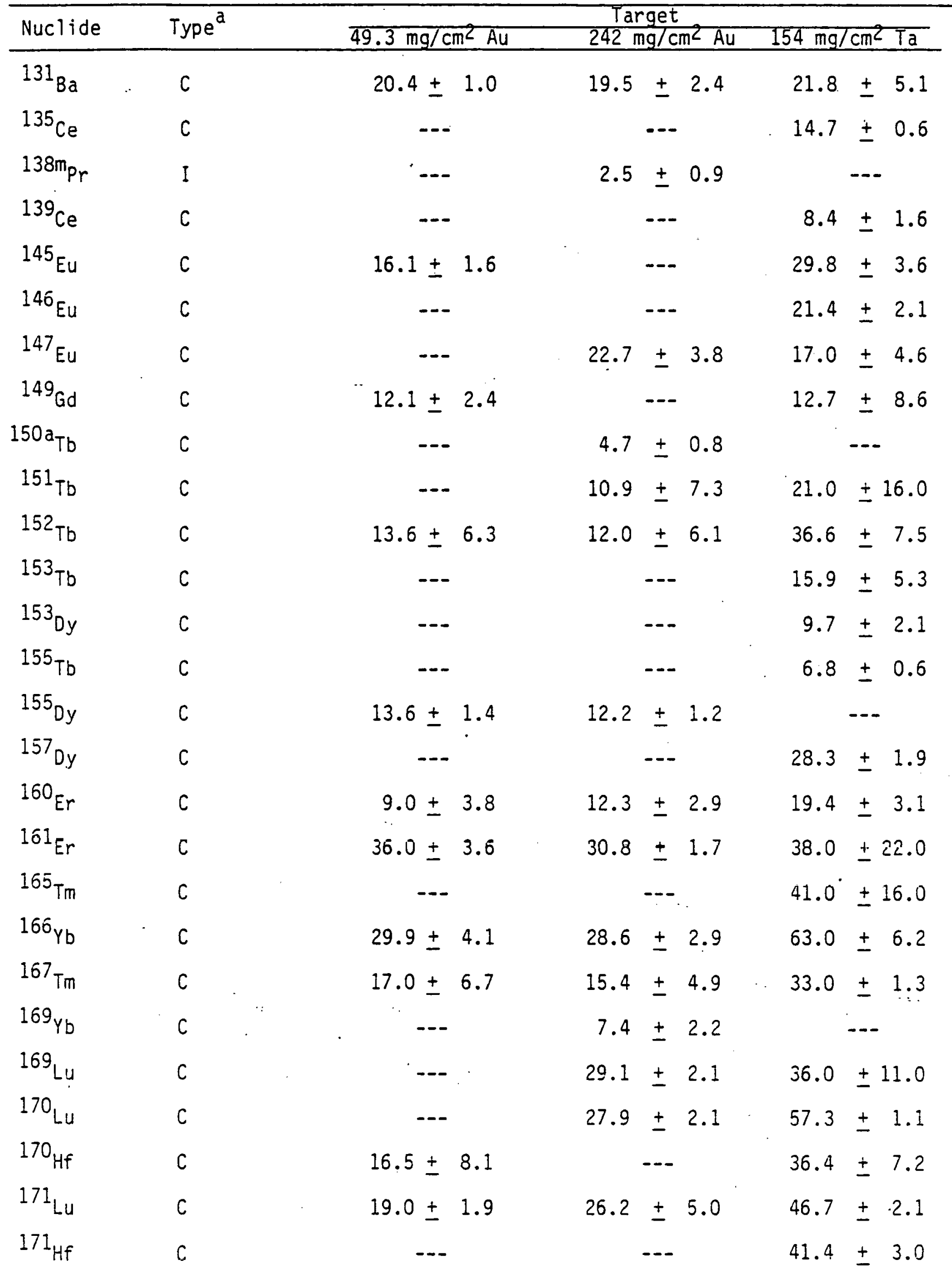


Table II. (continued)

\begin{tabular}{|c|c|c|c|c|c|c|c|}
\hline Nuclide & $\operatorname{TVne}^{a}$ & \multicolumn{6}{|c|}{ Target } \\
\hline wuctioe & & $49.3 \mathrm{mg} / \mathrm{cm}^{2} \mathrm{Au}$ & 242 & $\mathrm{ng} / \mathrm{cm}^{2} \mathrm{Au}$ & $154 \mathrm{mg} /$ & $1 \mathrm{~cm}^{2}$ & $\mathrm{Ta}$ \\
\hline${ }^{172} \mathrm{Lu}$ & I & -- & & --- & 11.1 & \pm & 1.4 \\
\hline $172_{\mathrm{Hf}}$ & C & --- & 24.5 & \pm 2.7 & & --- & \\
\hline $173_{\mathrm{HF}}$ & c & --- & & --- & 32.8 & \pm & 8.6 \\
\hline${ }^{173} \mathrm{Ta}$ & c & $12.0 \pm 7.0$ & 9.0 & \pm 2.8 & & -- & \\
\hline${ }^{174} \mathrm{Ta}$ & C & --- & 21.1 & \pm 6.4 & & --- & \\
\hline $175_{\mathrm{Hf}}$ & C & $--=$ & & - & 59.5 & \pm & 5.7 \\
\hline${ }^{176} \mathrm{Ta}$ & c & $52.0 \pm 25.0$ & 53.0 & \pm 20.0 & 55.4. & \pm & 2.2 \\
\hline${ }^{177} \mathrm{Ta}$ & C & -- & & -- & 16.8 & \pm & 1.7 \\
\hline $181_{\mathrm{Re}}$ & c & $42.4 \pm 8.0$ & 39.1 & $\pm \quad 3.8$ & & -- & \\
\hline${ }^{183} \mathrm{Re}$ & c & -- & 53.0 & \pm 18.0 & & --- & \\
\hline${ }^{184}$ Ir & c & $18.9 \pm 7.0$ & 13.0 & \pm 4.5 & & -- & \\
\hline${ }^{185} \mathrm{Ir}$ & C & $29.2 \pm 3.0$ & & -- & & --- & \\
\hline${ }^{187}$ Ir & C & --- & 58.0 & \pm 17.0 & & -- & \\
\hline${ }^{189}$ Ir & C & $42.6 \pm 4.1$ & 41.3 & \pm 3.6 & & --- & \\
\hline${ }^{190} \mathrm{Ir}$ & I & $5.5 \pm 0.4$ & 5.0 & \pm 0.46 & & $=--$ & \\
\hline${ }^{192} \mathrm{Ir}$ & I & --- & 2.8 & \pm 0.2 & & --- & \\
\hline${ }^{188} \mathrm{Pt}$ & C & $32.4 \pm 3.0$ & 29.8 & \pm 2.8 & & -- & \\
\hline $191_{P+}$ & c & --- & 00.6 & \pm 9.2 & & $\cdots$ & \\
\hline${ }^{191_{\mathrm{Au}}}$ & C & $31.7 \pm 2.8$ & & --- & & -- & \\
\hline${ }^{194} \mathrm{Au}$ & I & --- & 56.0 & \pm 3.8 & & -- & \\
\hline${ }^{196} \mathrm{Au}$ & $\mathrm{t}$ & $174.0 \pm 25.0$ & 181.9 & \pm 14.4 & & --- & \\
\hline${ }^{198} \mathrm{Au}$ & I & $43.1 \pm 3.8$ & 16.6 & \pm 0.8 & & --- & \\
\hline
\end{tabular}

a) Nuclides are typed I or $C$ to indicate either independent yield or partial cumulative yield, respectively. This distinction is discussed in the text. 
Table III. Thick to thin target cross section ratio from the 197 Au targets.

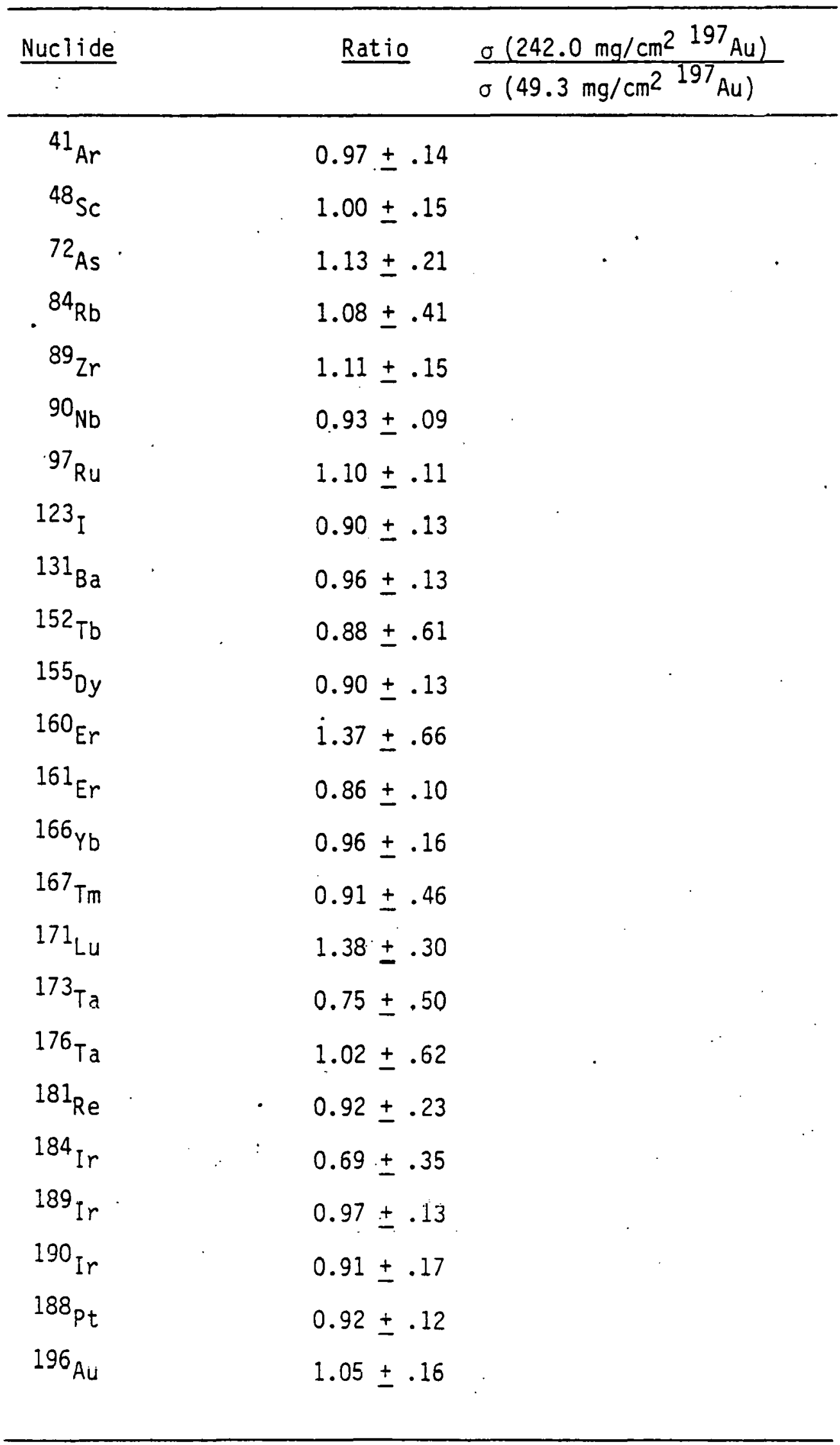


Table IV. Charge dispersion parameters.

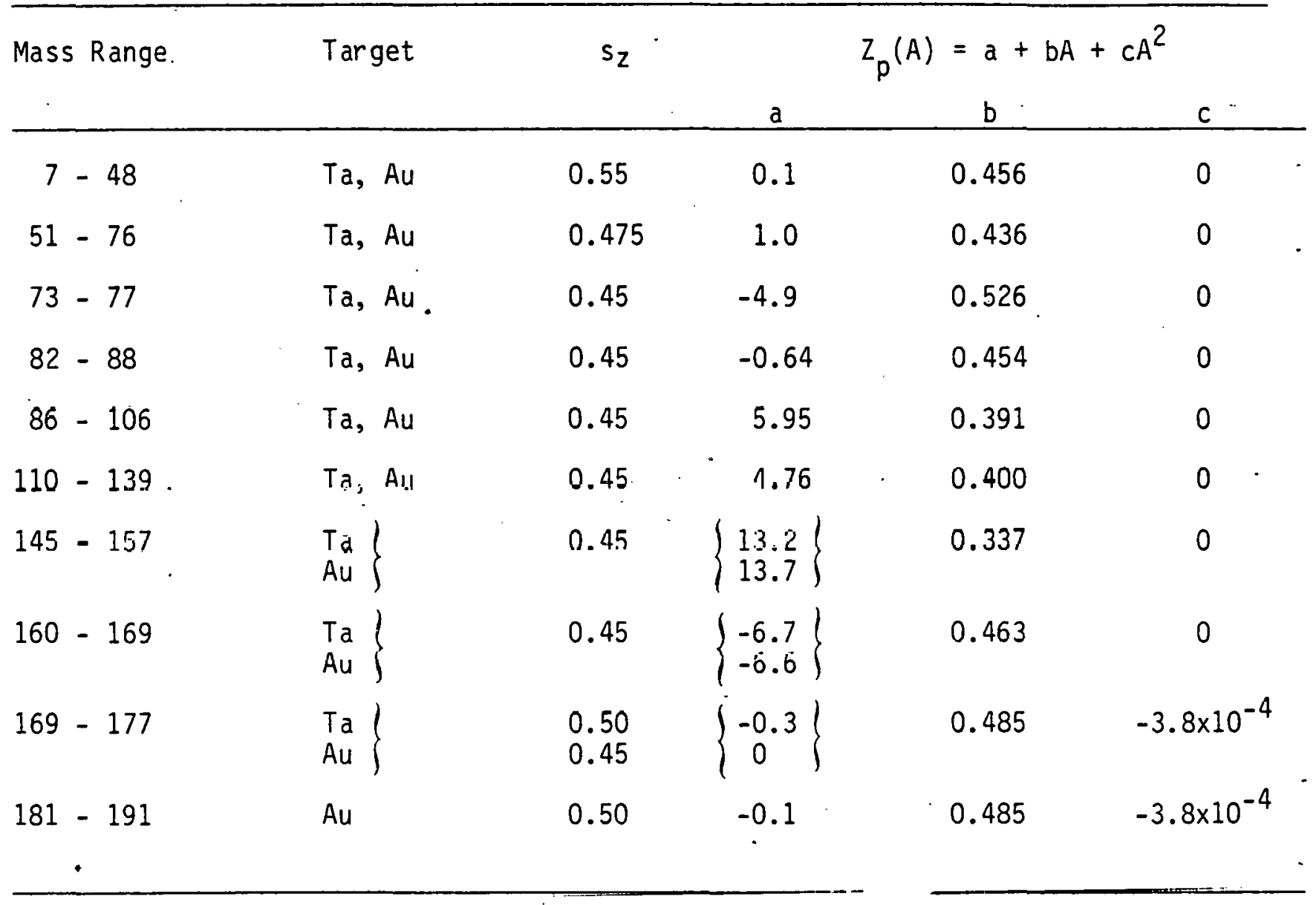


Table V. Total cross section comparison.

\begin{tabular}{cccc}
\hline & $\begin{array}{c}\text { Experimental } \\
\text { Large Fragment Cross Section } \\
\text { (barns) }\end{array}$ & \multicolumn{2}{c}{$\begin{array}{c}\text { Calculated Cross Section } \\
\text { (barns) }\end{array}$} \\
\hline $20 \mathrm{Ne}+{ }^{181} \mathrm{Ta}$ & $2.8 \pm 0.5$ & 3.64 & 3.47 \\
$20 \mathrm{Ne}+{ }^{197} \mathrm{Au}$ & $2.8 \pm 0.5$ & 3.80 & 3.59 \\
\hline
\end{tabular}




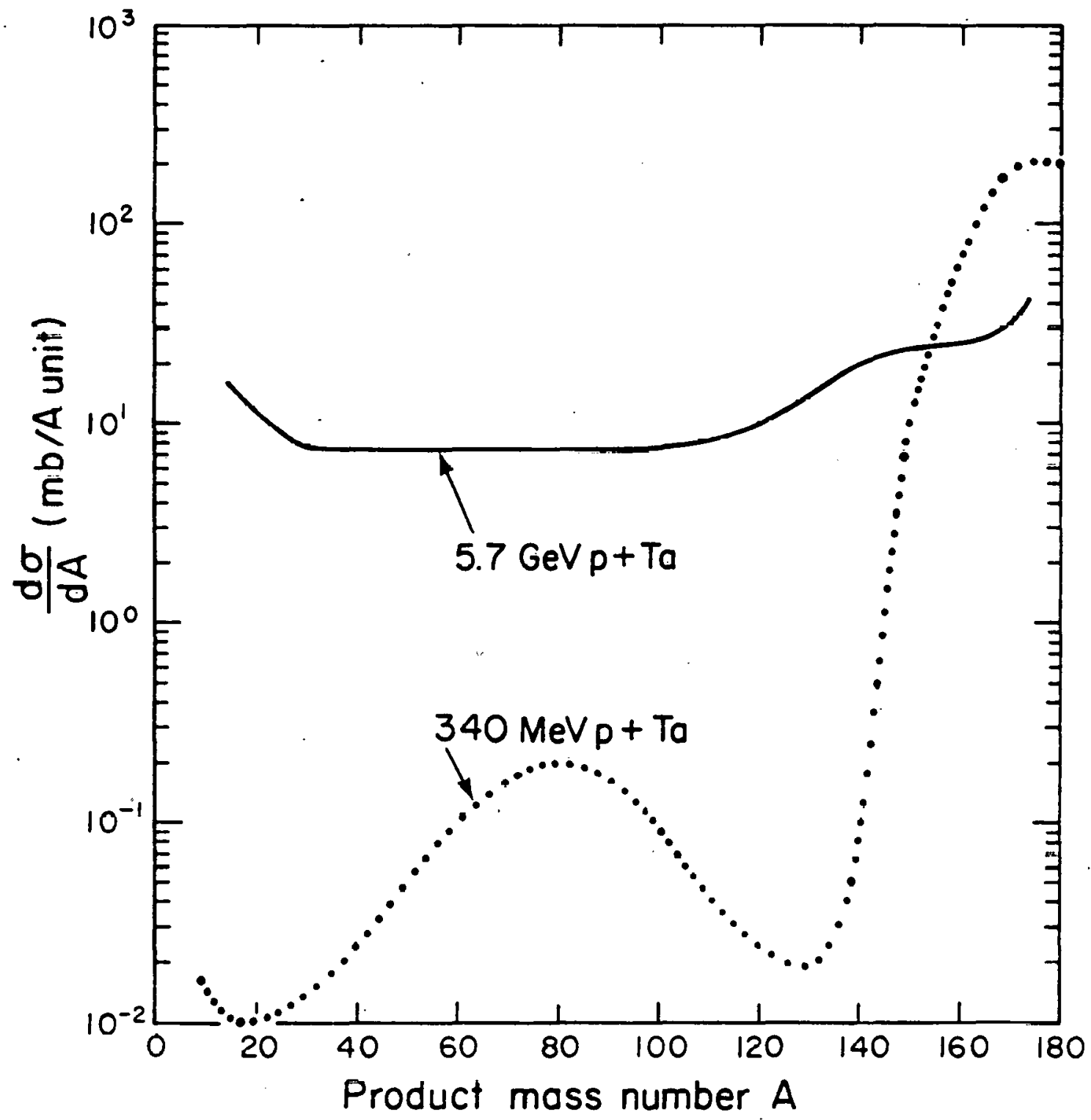

$X 8 L 794-1083$ 

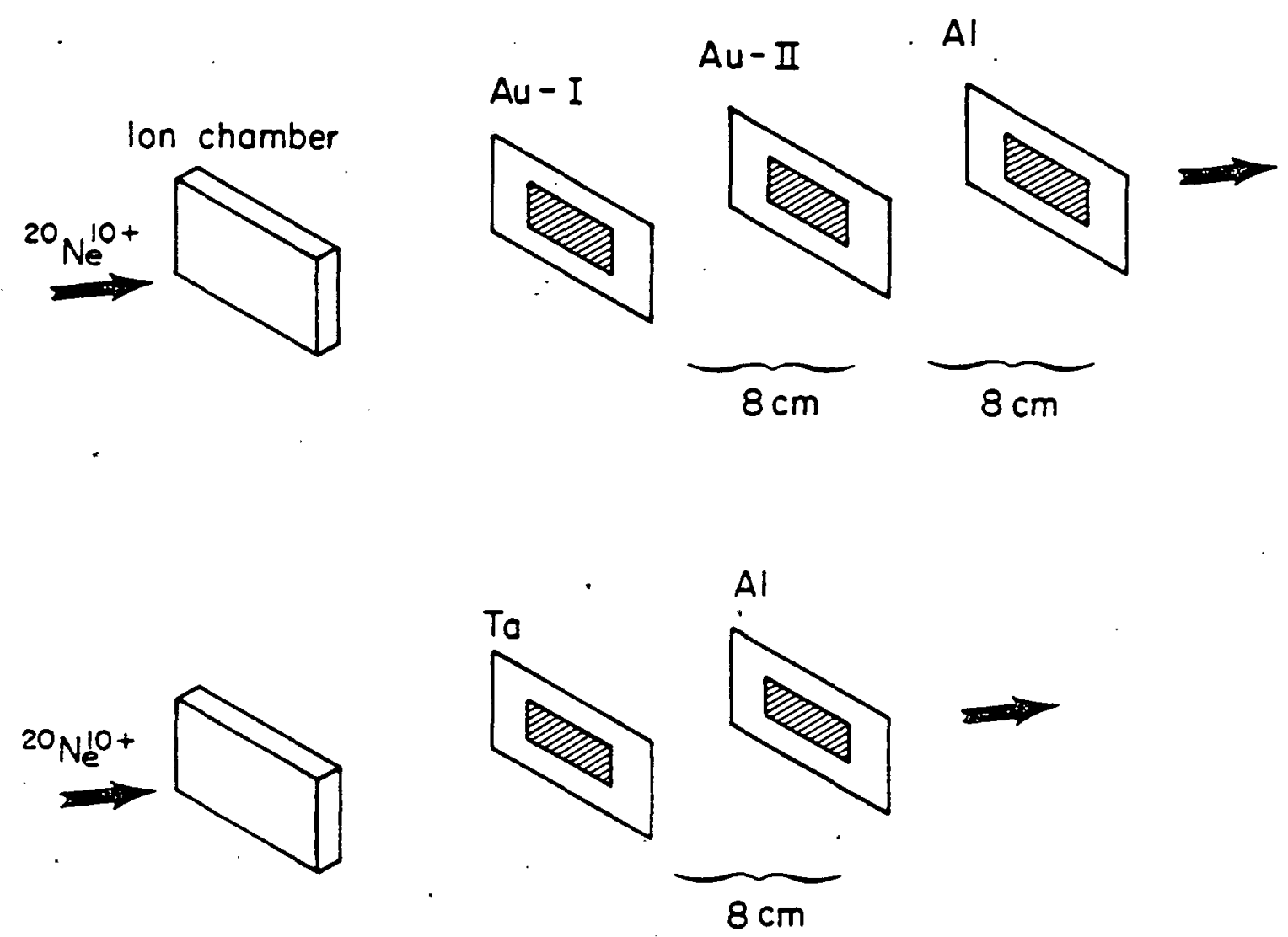

XBL 794-1079 


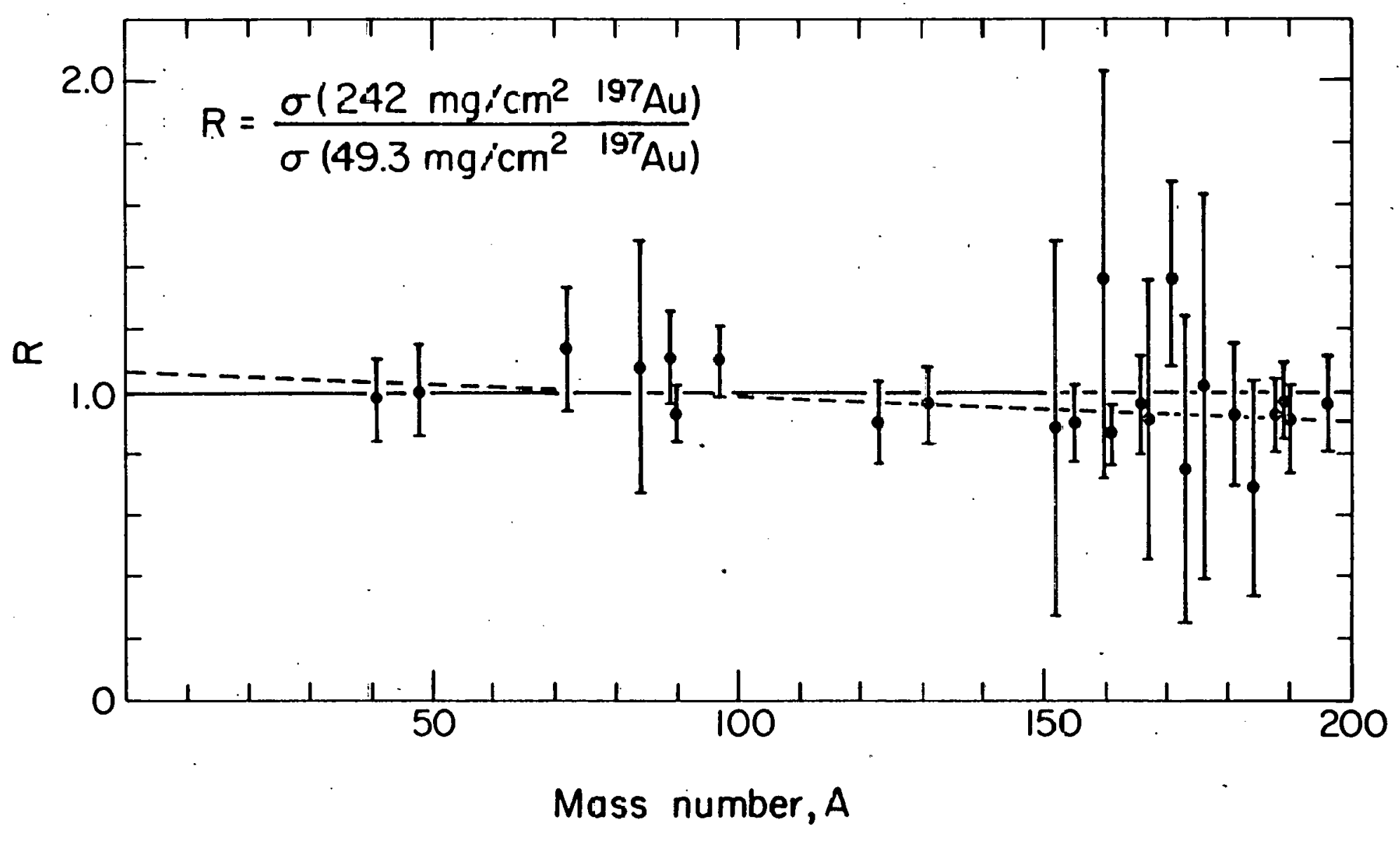




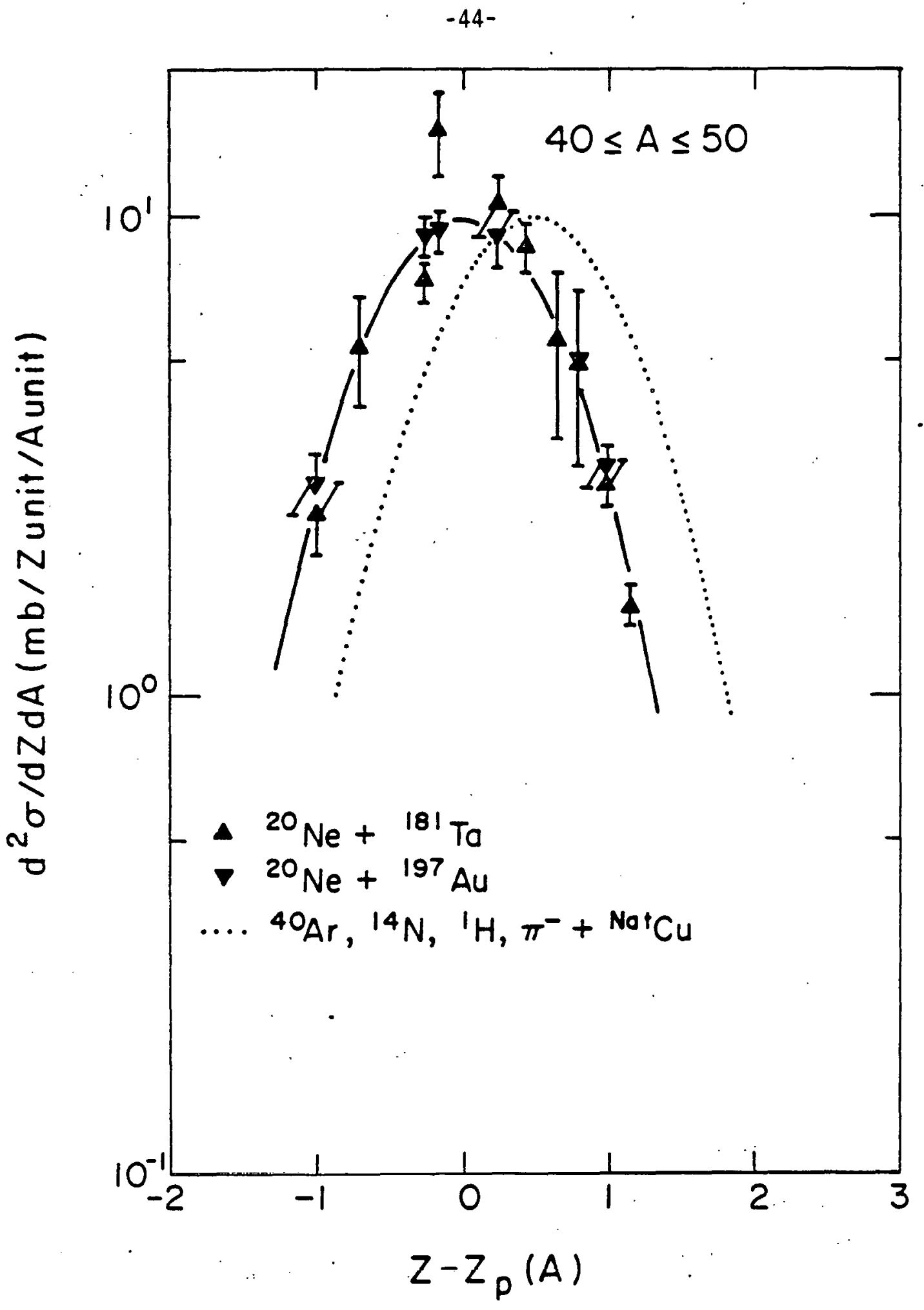

XBL 794-1082 
$-45-$

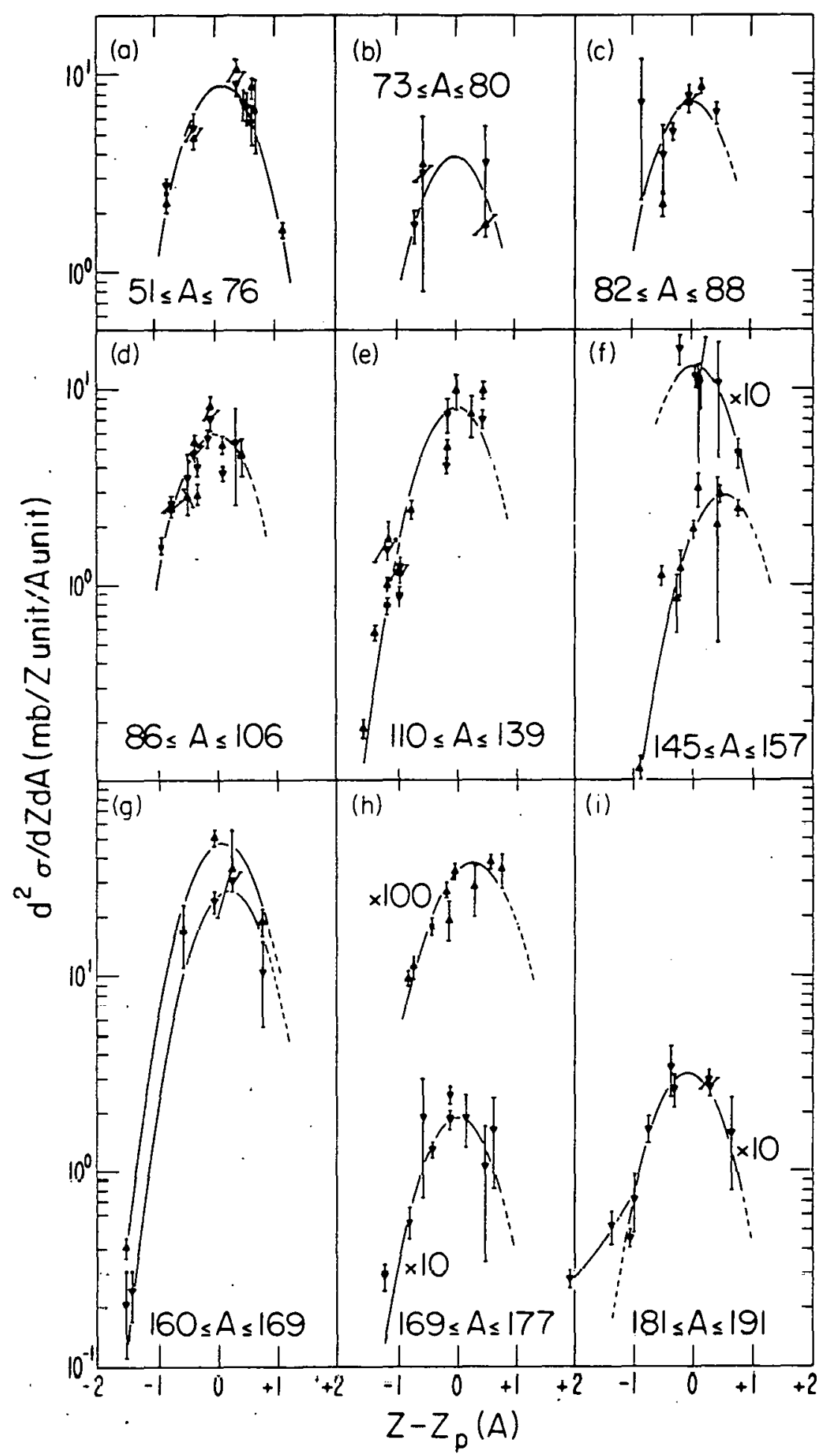

X6L $794-1078$ 


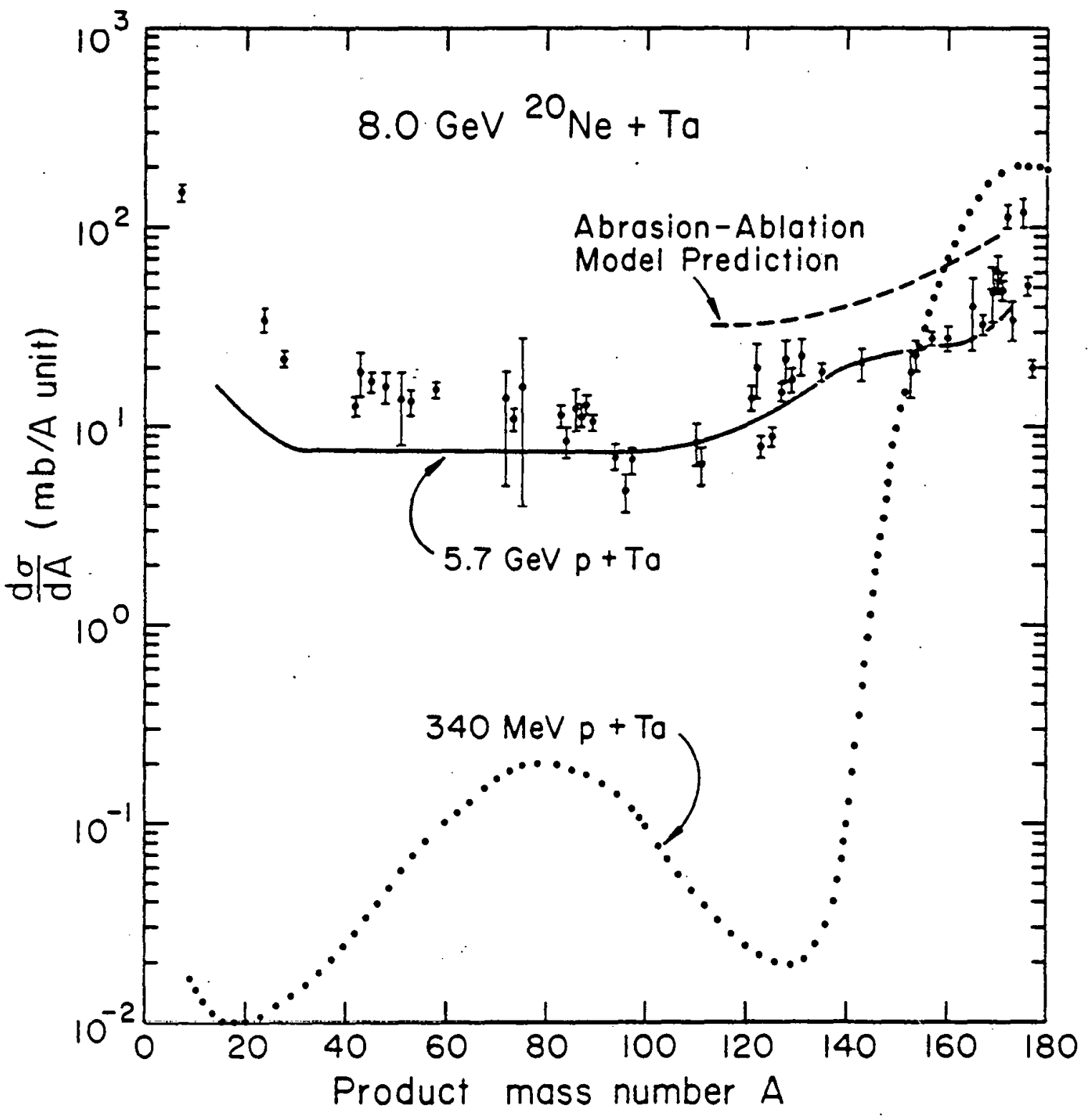




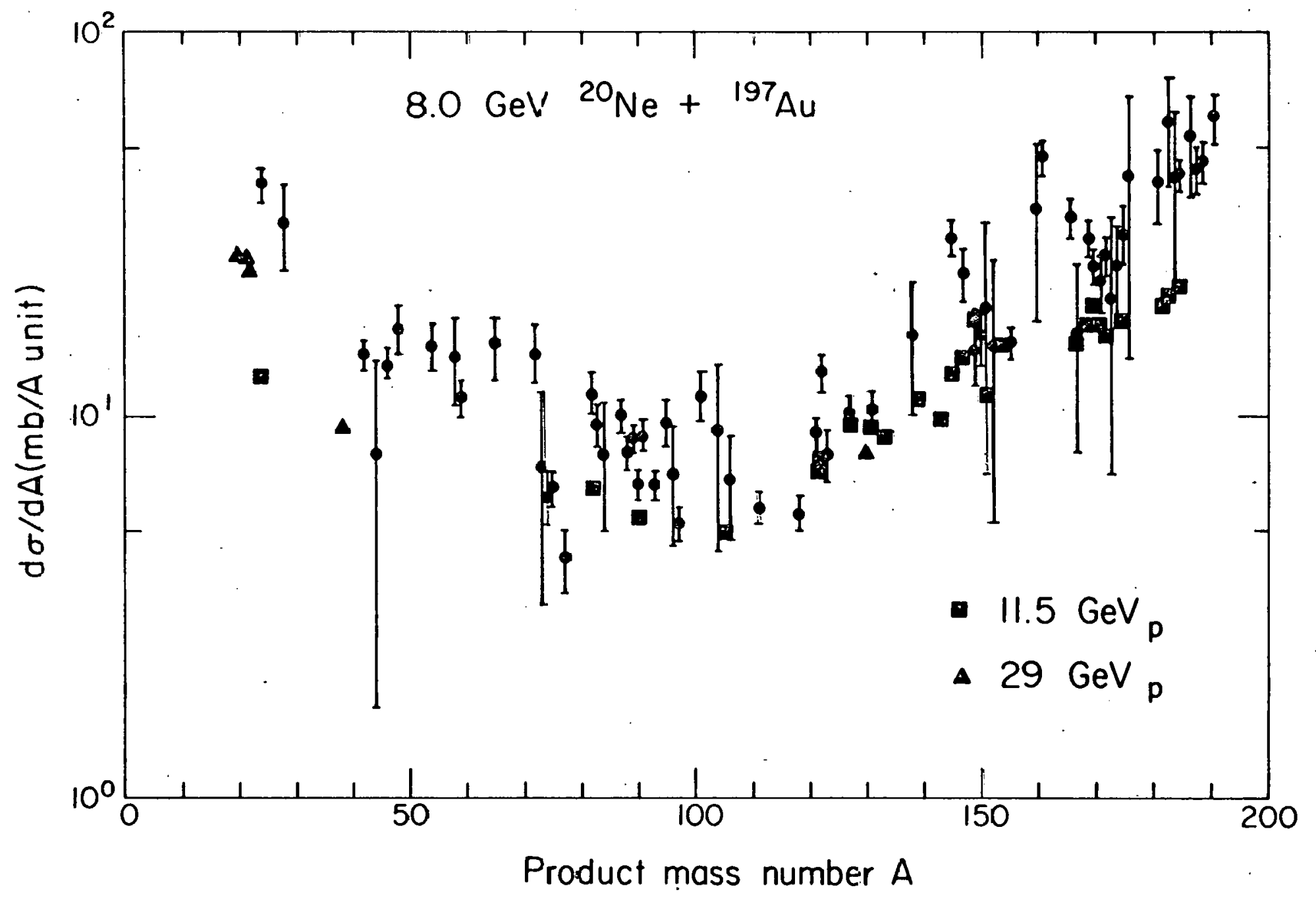




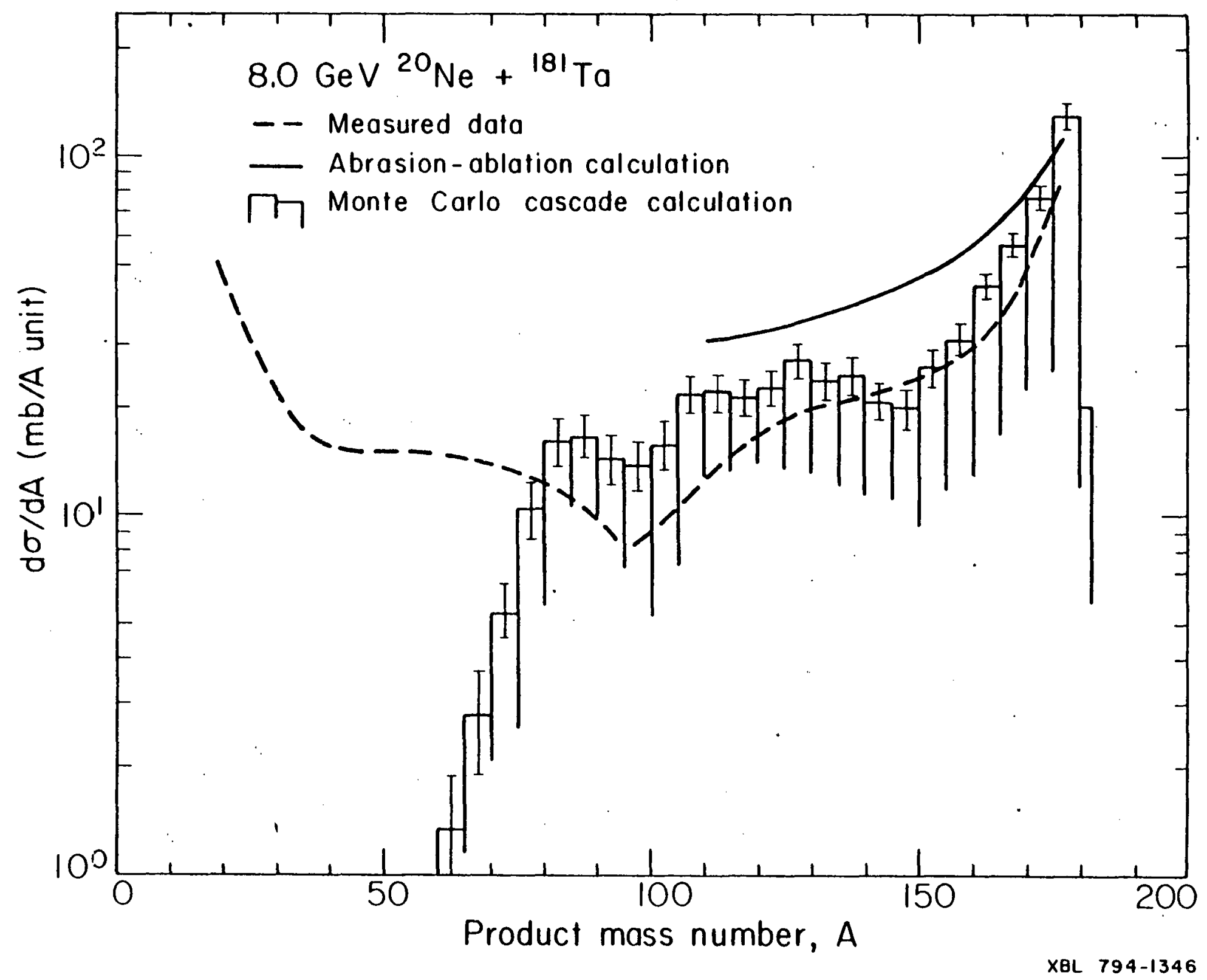




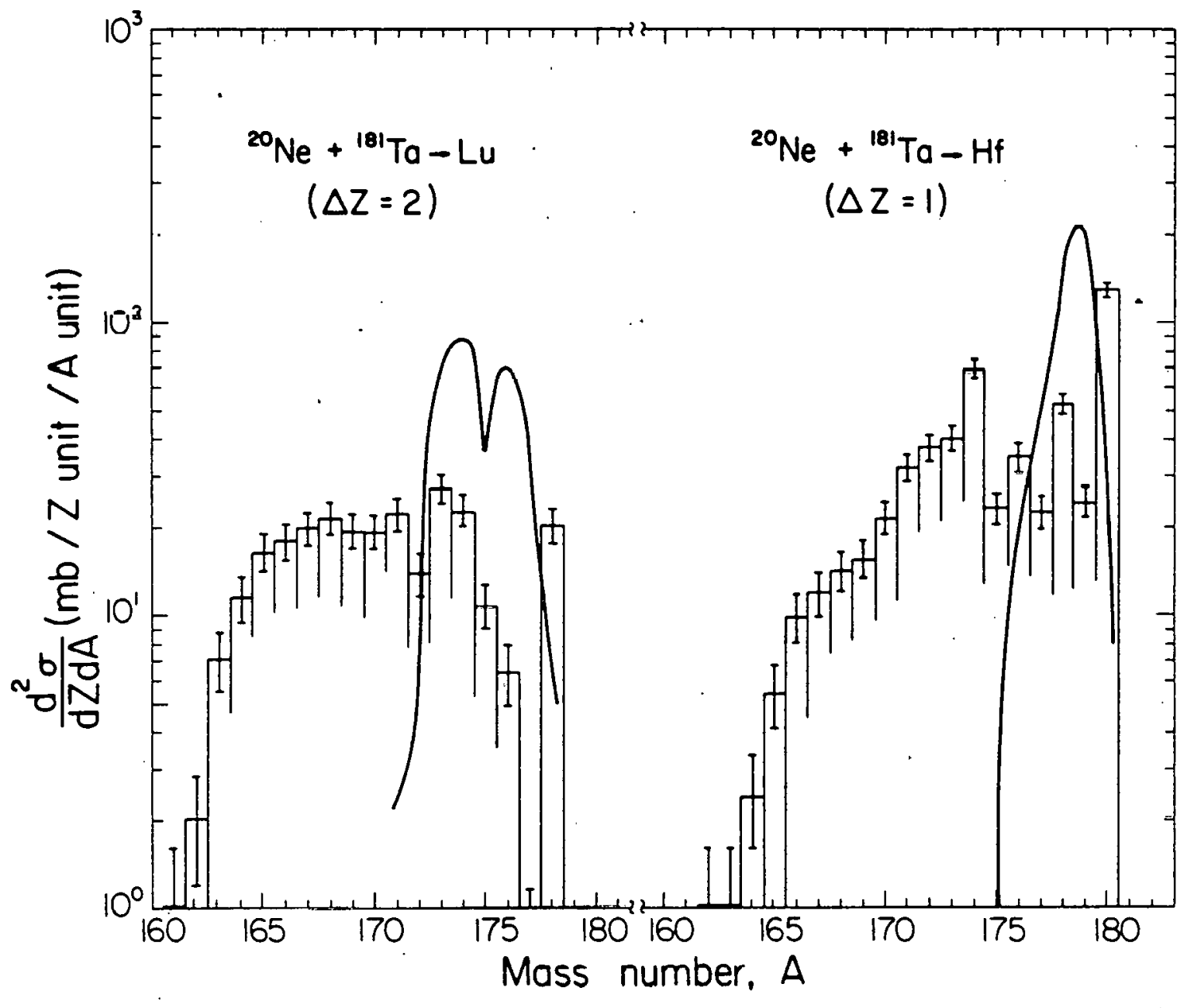

XQL $793-898$ 


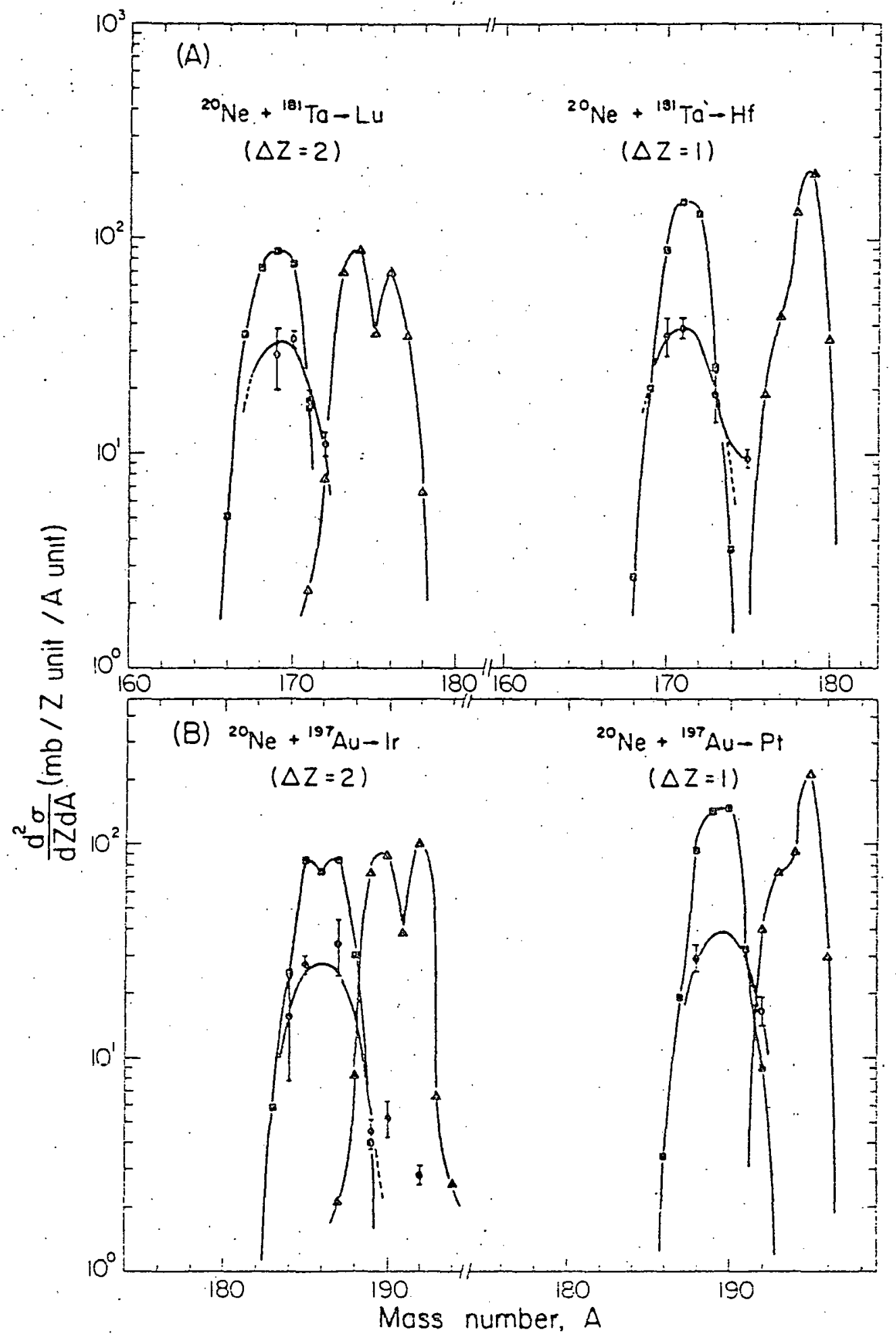

